\title{
A magnetic instability of the non-Abelian Sakai-Sugimoto model
}

\author{
Nele Callebaut ${ }^{a, b}$ and David Dudal ${ }^{a}$ \\ ${ }^{a}$ Ghent University, Department of Physics and Astronomy, \\ Krijgslaan 281-S9, 9000 Gent, Belgium \\ ${ }^{b}$ Theoretische Natuurkunde, Vrije Universiteit Brussel and \\ International Solvay Institutes, \\ Pleinlaan 2, B-1050 Brussels, Belgium \\ E-mail: ncalleba.callebaut@ugent.be, david.dudal@ugent.be
}

ABSTRACT: In this follow-up paper of [1] we further discuss the occurrence of a magnetically induced tachyonic instability of the rho meson in the two-flavour Sakai-Sugimoto model, uplifting two remaining approximations in the previous paper. That is, firstly, the magnetically induced splitting of the branes is now taken into account, evaluating without approximations the symmetrized trace which enters in the non-Abelian Dirac-Born-Infeld (DBI) action. This leads to an extra mass generating effect for the charged heavy-light rho meson through a holographic Higgs mechanism. Secondly, we compare the results in the approximation to second order in the field strength to the results using the full DBIaction. Both improvements cause an increase of the critical magnetic field for the onset of rho meson condensation. In addition, the stability in the scalar sector in the presence of the magnetic field is discussed.

KEYwORDs: Gauge-gravity correspondence, Phase Diagram of QCD, QCD

ARXIV EPRINT: 1309.5042 


\section{Contents}

1 Introduction 1

2 Goal and strategy $\quad 3$

3 Set-up $\quad \mathbf{5}$

3.1 Review of the Sakai-Sugimoto model 5

$\begin{array}{lll}3.2 & \text { Numerical fixing of the holographic parameters } & 7\end{array}$

3.3 Non-Abelian probe brane action 8

3.4 Effect of uniform magnetic field on the probe branes' embedding 8

4 Stability analysis $\quad 11$

$\begin{array}{ll}4.1 \text { Gauge fixing } & 13\end{array}$

$\begin{array}{lll}\text { 4.1.1 STr-evaluation } & 13\end{array}$

$\begin{array}{lll}\text { 4.1.2 Choosing a 't Hooft gauge } & 14\end{array}$

$\begin{array}{ll}\text { 4.1.3 Fixing the remaining gauge freedom } & 15\end{array}$

$\begin{array}{lll}4.2 & \text { Stability in scalar sector } & 16\end{array}$

$\begin{array}{ll}4.3 \text { Vector sector in }\left(2 \pi \alpha^{\prime}\right)^{2} F^{2} \text {-approximation } & 19\end{array}$

4.3.1 Rho meson mass and rho meson condensation 20

$\begin{array}{ll}\text { 4.3.2 Pion mass } & 27\end{array}$

4.4 Vector sector for full DBI-action 30

4.4.1 Comments on the validity of the $\left(2 \pi \alpha^{\prime}\right)$-expansion 30

4.4.2 Deriving the effective 4-dimensional equations of motion 31

4.4.3 Solving the 4-dimensional equations of motion 33

$\begin{array}{lll}4.5 & \text { Comment on the antipodal case } & 38\end{array}$

$\begin{array}{lll}5 & \text { Summary } & 40\end{array}$

A STr-prescription $\quad \mathbf{4 2}$

A.1 Derivation of the prescription 42

\section{Introduction}

The Sakai-Sugimoto model $[2,3]$ is one of the most used holographic QCD-models to study effective low-energy effects of a QCD-like theory at strong coupling. Its main merits are the incorporation of spontaneous chiral symmetry breaking, closely related to the description of confinement in the model, and the fact that previously constructed effective low-energy QCD models (such as the Skyrme model for pions, the hidden local symmetry approach for the coupling of pions and rho mesons, vector meson dominance for the pion formfactor, etc.) drop out automatically. 
In this paper we further investigate the stability of the two-flavour Sakai-Sugimoto model in the presence of a magnetic field, and this in the confinement phase. We will find stability in the scalar and an instability in the charged vector sector. Previous stability analyses of the Sakai-Sugimoto model (SSM) have mainly focused on the case of a background chemical potential. In particular Chern-Simons-induced instabilities to spatially modulated phases have received quite some attention recently [4-9]. Earlier works in this context include [10-12], and $[13,14]$ on the $(T, \mu, B)$ phase diagram in the Sakai-Sugimoto model. More relevant for our current purposes is the DBI-induced instability in the presence of an isospin chemical potential studied in [15], where a tachyonic instability of the rho meson and ensuing rho meson condensation was described. We will encounter a somewhat similar phenomenon here, but as a result of the presence of a background magnetic field $B$ and zero chemical potential.

The papers referred to above which include magnetic fields, use the original antipodal SSM in which the flavour branes are positioned $B$-independently at opposite points on the supersymmetry-breaking circle of the background. We will focus on the more general nonantipodal embedding of flavour branes, in which case the embedding does depend on the magnetic field, corresponding to chiral magnetic catalysis in the dual field theory [16-19].

The stability of the embedding of the flavour branes has been checked in [2] for the antipodal case, and in $[20,21]$ for the non-antipodal case. We extend this analysis to the non-antipodal, $B$-dependent embedding, finding what we referred to as 'stability in the scalar sector' earlier.

We believe we are also the first to consider multiple non-antipodal embedded flavour branes $N_{f}>1$ that couple to the external magnetic field with different electric charges, modeling differently charged up- and down-quarks. Taking this complication into account will create a magnetically induced splitting of the flavour branes, interpreted as explicit breaking of the $\mathrm{U}\left(N_{f}\right)$ chiral symmetry to a product of Abelian $\mathrm{U}(1)$ chiral symmetries, which makes the evaluation of the symmetrized trace in the action significantly more cumbersome.

In the end, we find a holographic description of the instability towards rho meson condensation in the presence of a very strong magnetic field, first discussed in phenomenological QCD-models in $[22,23]$. This is one of the many effects studied recently in the context of QCD in extreme conditions, a research area that has naturally gained more interest with the growing availability of data on quark-gluon plasma from LHC and RHIC experiments. There, not only high temperatures and high densities are present, but also, when the plasma is created in non-central heavy ion collisions, very high magnetic fields (of the order of $10^{15}$ Tesla) [24-29]. For a review on strongly interacting matter in magnetic fields, see [30] and references therein.

Many magnetic effects have been investigated in the Sakai-Sugimoto model, so, to avoid incompleteness, let us refer here to the review paper [31] for a nice overview. 


\section{Goal and strategy}

Basic argument for rho meson condensation in field theory. In [22], a possible magnetic instability of the QCD vacuum towards a phase where charged rho mesons are condensed is discussed. The basic argument for this rho meson condensation at some critical value of the magnetic field $B_{c}$, is that the charged rho meson combinations which have their spin aligned with the magnetic field $B$, have an effective mass squared

$$
m_{\rho, e f f}^{2}(B)=m_{\rho}^{2}-e B
$$

which vanishes at

$$
e B_{c}=m_{\rho}^{2}=0.602 \mathrm{GeV}^{2},
$$

based on the fact that the $n$-th energy level of a free, structureless spin- $s$ particle with mass $m$ in the presence of a background magnetic field $\vec{B}=B \vec{e}_{3}$ is given by the wellknown Landau level quantization formula

$$
E^{2}=m_{\rho}^{2}+p_{3}^{2}+\left(2 n-2 s_{3}+1\right) e B
$$

with $p_{3}$ the particle's momentum in the direction of the magnetic field, and $s_{3}$ its spin projection on the same direction. This leads to (2.1) for the lowest-energy rho meson $p_{3}=0$, $n=0$ with $\operatorname{spin} s_{3}=1$.

The above argument holds in the context of the bosonic effective DSGS-model [32] for rho meson quantum electrodynamics, used in [22]. Somewhat later, the rho meson condensation effect was also shown to emerge in the NJL-model [23]. It should be clear however that rho meson condensation is merely conjectured to occur in QCD based on these descriptions in effective QCD-models, not proven nor experimentally observed. To date, the effect of rho meson condensation has been discussed in [22, 23, 33-35] using phenomenological and lattice approaches, in our work [1] using the Sakai-Sugimoto model, and in [36-38] using a bottom-up holographic approach. Its possible occurrence has been argued against in [39] — followed by a rebuttal in [40, 41] showing that the counterarguments of [39] should not apply.

Goal. Our goal is to study the effective rho meson mass squared $m_{\rho, \text { eff }}^{2}(B)$ in a full-blown holographic top-down approach, using the Sakai-Sugimoto model. In a simplified set-up, we were able to show in [1] that rho meson condensation does occur in this model. The $B$-dependence of the rho meson mass will be further investigated here, thereby uplifting remaining approximations in [1]. The influence of chiral magnetic catalysis on the differently charged constituents of the mesons is taken into account by considering the non-antipodal embedding. This will lead to a modification of the energy levels (2.3). We shall however continue to use the nomenclature Landau levels. The instability is still present, at a somewhat higher value of $e B_{c}$ than the estimate (2.2). We focus on the confinement phase of the model and set the number of flavours equal to two, $N_{f}=2$, necessary to describe charged mesons. 
Outline. We start with an outline of the set-up in section 3, including a short review of the Sakai-Sugimoto model. We fix the number of colours $N_{c}=3$ and the rest of the holographic parameters to numerical $\mathrm{GeV}$ units, in order to obtain results for $m_{\rho, \text { eff }}^{2}$ and $B_{c}$ in physical units, comparable to other - phenomenological and lattice - approaches. In the same section, the effect of the magnetic field on the probe branes' embedding is reviewed.

In section 4 we discuss the stability of the fluctuations. For that purpose we plug a flavour gauge field ansatz containing a background $(\sim B)$ and a fluctuation part $(\sim$ mesons) into the non-Abelian DBI-action governing the dynamics of the flavour gauge field living on the probe branes, and expand the action to second order in the fluctuations. The eventual goal is to extract the effective rho meson mass from the 4-dimensional mass equation for the vector meson, the effective 4-dimensional action to be obtained from the DBI-action by integrating out the extra dimensions.

First, we have to choose a particular gauge to disentangle the scalar and vector fluctuations in the action, this is done in section 4.1. Then we discuss the stability with respect to scalar fluctuations, corresponding to the positions of the probe branes. Next, we consider the vector fluctuations. This we already partly covered in our previous paper [1], where we discussed the case of antipodal embedding and the case of non-antipodal embedding with the action approximated to second order in the total field strength $F$ and with the extra assumption of coinciding branes. Here, we extend on these analyses by considering the non-antipodal embedding with magnetically separated branes, both in the case of using the action expanded to second order in $F$ (section 4.3) and the full non-linear DBI-action in $F$ (section 4.4). Because the field strength $F$ in the DBI-action is accompanied with a factor proportional to the inverse of the 't Hooft coupling $\lambda$, which is large in the validity range of the gauge-gravity duality, the expansion to second order in $F$ is commonly used. However, in the presence of large background fields, the higher order terms may become important (see section 4.4.1). We therefore compare the outcome of using the $F^{2}$-approximated action versus the full DBI-action, from which we can conclude that the difference in $B_{c}$ is very small and the $F^{2}$-expansion was justified in our case after all.

In section 4.3 the focus is on handling the magnetically separated branes. For noncoinciding branes, the symmetrized trace (STr) over flavour indices in the DBI-action no longer simplifies to a normal Tr. Instead, evaluating the STr (which can be done exactly to second order in the fluctuations) gives rise to complicated functions in the action (defined via integrals), which depend on the background fields and are discontinuous in the holographic radius $u$. We pay some attention to solving the eigenvalue equation for the rho meson eigenfunction with these functions present. The evaluation of the STr is discussed in section 4.1.1, with the used - exact - prescriptions outlined in the appendix, including a sketch of their derivation. In section 4.3.2, for completeness, we briefly discuss the pions in the DBI-action. The section ends with a comment on the validity of the use of the non-Abelian DBI-action for non-coincident branes.

In section 4.4 the focus is on handling the extra dependences on the magnetic field from considering the full DBI-action. The resulting effective 4-dimensional equation of motion (EOM) (to second order in the rho meson fields) has extra terms compared to the standard Proca EOM used in phenomenological descriptions of the rho meson in a background 
magnetic field, making it harder to analyze. We solve the EOMs for the complete energy spectrum exactly in section 4.4.3, with the main result for the generalized Landau levels given in eq. (4.131). The energy eigenstates are no longer spin eigenstates (as opposed to the Proca energy eigenstates), except for the condensing state.

We comment on the antipodal set-up with full DBI-action in section 4.5 and summarize in section 5 .

\section{Set-up}

\subsection{Review of the Sakai-Sugimoto model}

The Sakai-Sugimoto model $[2,3]$ is a holographic QCD-model, involving $N_{f}$ pairs of D8- $\overline{\mathrm{D} 8}$ flavour probe branes placed in a D4-brane background

$$
\begin{aligned}
d s^{2} & =g_{m n} d x^{m} d x^{n} \quad(m, n=0 \cdots 9) \\
& =\left(\frac{u}{R}\right)^{3 / 2}\left(\eta_{\mu \nu} d x^{\mu} d x^{\nu}+f(u) d \tau^{2}\right)+\left(\frac{R}{u}\right)^{3 / 2}\left(\frac{d u^{2}}{f(u)}+u^{2} d \Omega_{4}^{2}\right), \\
e^{\phi} & =g_{s}\left(\frac{u}{R}\right)^{3 / 4}, \quad F_{4}=\frac{N_{c}}{V_{4}} \epsilon_{4}, \quad f(u)=1-\frac{u_{K}^{3}}{u^{3}},
\end{aligned}
$$

where $d \Omega_{4}^{2}, \epsilon_{4}$ and $V_{4}=8 \pi^{2} / 3$ are, respectively, the line element, the volume form and the volume of a unit four-sphere, while $R$ is a constant parameter related to the string coupling constant $g_{s}$, the number of colours $N_{c}$ and the string length $\ell_{s}$ through $R^{3}=\pi g_{s} N_{c} \ell_{s}^{3}$. This background has a natural cut-off at $u=u_{K}$ and is therefore dual to a confining QCD-like theory, living on the boundary at $u \rightarrow \infty$. Imposing a smooth cut-off of space at $u=u_{K}$ uniquely determines the period $\delta \tau$ of $\tau$ :

$$
\delta \tau=\frac{4 \pi}{3} \frac{R^{3 / 2}}{u_{K}^{1 / 2}}=2 \pi M_{K}^{-1}
$$

with $M_{K}$ the inverse radius of the $\tau$-circle.

The parameters $R, g_{s}, \ell_{s}, M_{K}, u_{K}$ and 't Hooft coupling $\lambda=g_{Y M}^{2} N_{c}$ are related through the following equations:

$$
R^{3}=\frac{1}{2} \frac{\lambda \ell_{s}^{2}}{M_{K}}, \quad g_{s}=\frac{1}{2 \pi} \frac{g_{Y M}^{2}}{M_{K} \ell_{s}}, \quad u_{K}=\frac{2}{9} \lambda M_{K} \ell_{s}^{2} .
$$

Since all physical results are independent of the choice of $\lambda \ell_{s}^{2}$, one can moreover impose, without loss of generality, that $\frac{2}{9} M_{K}^{2} \ell_{s}^{2}=\frac{1}{\lambda}[3]$ which is the same as stating that

$$
u_{K}=\frac{1}{M_{K}} .
$$

Consequently, the remaining parameter relations reduce to

$$
R^{3}=\frac{9}{4} \frac{1}{M_{K}^{3}} \quad \text { and } \quad \frac{1}{g_{s} \ell_{s}^{3}}=\frac{4 \pi}{9} N_{c} M_{K}^{3}=\frac{4 \pi}{3} M_{K}^{3} .
$$



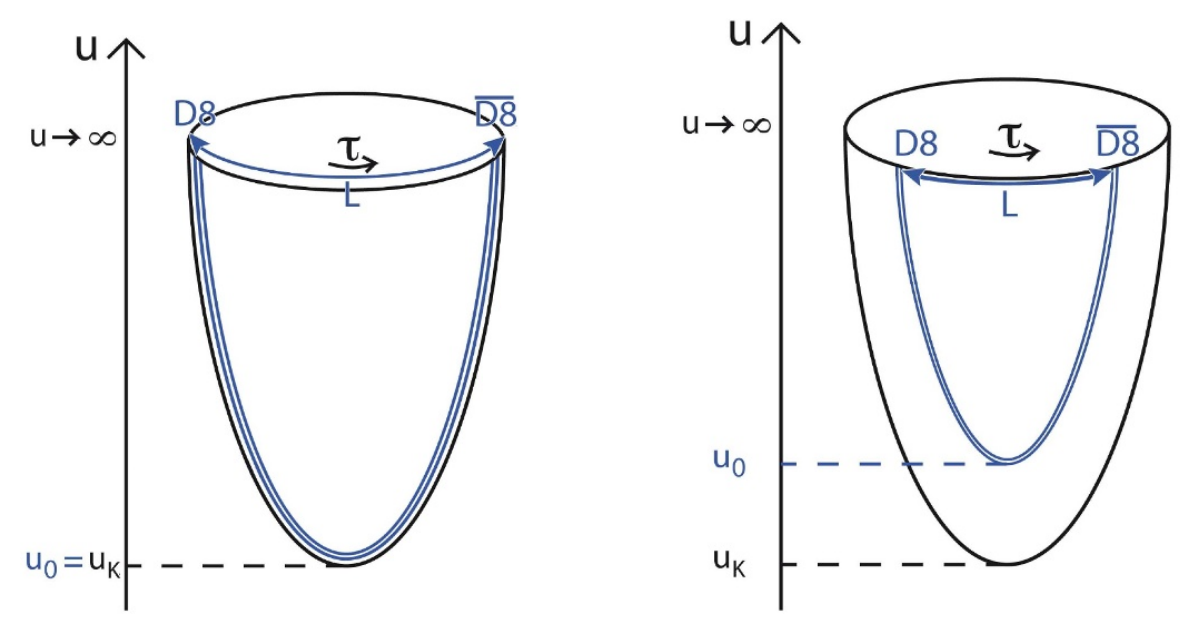

Figure 1. The Sakai-Sugimoto model: antipodal $\left(u_{0}=u_{K}\right)$ and non-antipodal $\left(u_{0}>u_{K}\right)$ embedding.

The duality is valid in the limit of a large number of colours $N_{c} \rightarrow \infty$ and large but fixed 't Hooft coupling $\lambda=g_{Y M}^{2} N_{c} \gg 1$ (with $g_{Y M} \rightarrow 0$ ), which means one probes the strong coupling regime of the 4-dimensional dual field theory in the 't Hooft limit. The backreaction of the $N_{f} \ll N_{c}$ flavour degrees of freedom on the D4-brane geometry is ignored. This is the so-called probe approximation [42] (or quenched approximation in QCD language). Furthermore, bare quark masses are zero, so this model is in the chiral limit. ${ }^{1}$

On the stack of $N_{f}$ coinciding D8- $\overline{\mathrm{D} 8}$ flavour pairs, there lives a $\mathrm{U}\left(N_{f}\right)_{L} \times \mathrm{U}\left(N_{f}\right)_{R}$ gauge theory for the flavour gauge field $A_{m}\left(x^{\mu}, u\right)(m=0,1,2,3, u)$ describing massless excitations of open strings attached to the branes. This gauge theory is interpreted as corresponding to the global chiral symmetry in the dual QCD-like theory. The cigar-shape of the $(u, \tau)$ subspace of the D4-brane background enforces a $\cup$-shaped embedding of the flavour branes, encoded in the embedding function $u(\tau)$. This particular form of the embedding represents the spontaneous breaking of chiral symmetry $\mathrm{U}\left(N_{f}\right)_{L} \times \mathrm{U}\left(N_{f}\right)_{R} \rightarrow \mathrm{U}\left(N_{f}\right)$ as the merging of the D8-branes and $\overline{\mathrm{D}} 8$-branes at $u=u_{0}$. The asymptotic separation $L($ at $u \rightarrow \infty)$ between D8- and $\overline{\mathrm{D}} 8$-branes, indicated in figure 1 , is related to $u_{0}$ as

$$
\begin{aligned}
L & =2 \int_{u_{0}}^{\infty} \frac{d u}{u^{\prime}} \quad\left(\text { with } u^{\prime}=d u / d \tau\right) \\
& =2 \int_{u_{0}}^{\infty} d u\left(\frac{R}{u}\right)^{3 / 2} f(u)^{-1} \sqrt{\frac{u_{0}^{8} f\left(u_{0}\right)}{u^{8} f(u)-u_{0}^{8} f\left(u_{0}\right)}}
\end{aligned}
$$

In the original set-up of $[2,3]$ the embedding is antipodal: the flavour branes merge at the tip of the cigar, $u_{0}=u_{K}$. In the more general non-antipodal embedding, $u_{0}>u_{K}$, the distance between $u_{K}$ and $u_{0}$ is interpreted to be related to the constituent quark mass as

\footnotetext{
${ }^{1}$ To overcome this, in [43] the bifundamental 'tachyon'-field connecting D8- and $\overline{\mathrm{D} 8}$-branes is taken into account. Other possible mechanisms to include bare quark masses can be found in $[44,45]$. We did not consider these options here for reasons of simplicity.
} 
the energy stored in a string stretching from $u_{0}$ to $u_{K}[46]$ :

$$
m_{q}=\frac{1}{2 \pi \alpha^{\prime}} \int_{u_{K}}^{u_{0}} \frac{d u}{\sqrt{f(u)}},
$$

with $2 \pi \alpha^{\prime}$ the inverse string tension, related to the string length through $\alpha^{\prime}=\ell_{s}^{2}$. In the latter set-up, unlike in the $u_{0}=u_{K}$ case, it is possible [16-19] to model the effect of chiral magnetic catalysis [47] which says that a magnetic field boosts the chiral symmetry breaking and hence the constituent quark masses. More precisely, the authors of [47] discuss a low-energy theorem in the context of chiral perturbation theory, thereby finding that the chiral condensate grows (linearly) in terms of an increasing magnetic field, with the coefficient a function of the pion decay constant $f_{\pi}$.

In this work, we will interpret $m_{q}$ as an indicator for the chiral symmetry breaking order parameter, for lack of a chiral condensate in the used set-up. Let us however remark that possible alternatives to define chiral order parameters can be found in, for example, [48] or $[43-45,49,50]$.

\subsection{Numerical fixing of the holographic parameters}

In this paper, for the purpose of presenting the end results in physical $\mathrm{GeV}$ units, we will fix the number of colours to three, $N_{c}=3$. We choose the number of flavours to be two, $N_{f}=2$, in order to be able to model electromagnetically charged mesons consisting of upand down quarks. This means we are stretching the validity of the probe approximation, but we will nonetheless ignore the backreaction. With these choices, we are then able to fix the remaining free parameters in the model, $R, \lambda, \ell_{s}, M_{K}, u_{0}, g_{s}$ and $L$, by matching to the following QCD input parameters

$$
m_{q}=0.310 \mathrm{GeV}, \quad f_{\pi}=0.093 \mathrm{GeV} \text { and } m_{\rho}=0.776 \mathrm{GeV}
$$

for resp. the constituent quark mass $m_{q}$, the pion decay constant $f_{\pi}$ and the $\rho$ meson mass $m_{\rho}$, in absence of magnetic field.

The results of our numerical analysis are (for the underlying details we refer to [1])

$$
M_{K} \approx 0.7209 \mathrm{GeV}, \quad \frac{u_{0}}{u_{K}} \approx 1.38 \text { and } \kappa=\frac{\lambda N_{c}}{216 \pi^{3}} \approx 0.006778
$$

From these values we do extract a relatively large 't Hooft coupling, $\lambda \approx 15$, and (via (3.6)) a value for the asymptotic flavour brane separation $L \approx 1.574 \mathrm{GeV}^{-1}$ that is approximately 2.8 times smaller than the maximum value of $L$, given by $L_{\max }=\frac{\delta \tau}{2}=$ $\frac{\pi}{M_{K}} \approx 4.358 \mathrm{GeV}^{-1}$. Our estimate for the effective string tension between a quark and an antiquark becomes $\sigma \approx 0.19 \mathrm{GeV}^{2}$, in excellent agreement with the pure $\mathrm{SU}(3)$ lattice-QCD value $\sigma \approx 0.18-0.19 \mathrm{GeV}^{2}[51,52]$. This means we could equally well have used the value for $\sigma$ as input, instead of $m_{q}$, as it could be noted that the identification in (3.7) is rather an indicator of magnitude than an exact correspondence.

Using the above values for the parameters enables us to present all our results in physical units, and in particular compare our result for the critical magnetic field for the onset of rho meson condensation to the values obtained in other (phenomenological or lattice) QCD approaches. 


\subsection{Non-Abelian probe brane action}

The dynamics of the stack of $N_{f}$ coinciding $\mathrm{D} 8-\overline{\mathrm{D} 8}$ flavour branes in the 10 -dimensional D4-brane background is determined by the dynamics of open strings with their endpoints attached to the branes. The spectrum of vibrational modes of these attaching strings contains a massless $\mathrm{U}\left(N_{f}\right)$ flavour gauge field with 10 components, which can be decomposed in a $\mathrm{U}\left(N_{f}\right)$ flavour gauge field $A_{m}\left(x^{\mu}, u\right)(m=0,1,2,3, u)$ living on the world volume of the branes (we set $A_{\Omega_{4}}=0$ and $\partial_{\Omega_{4}} A_{m}=0$ ) and a scalar field $\tau$ describing fluctuations of the branes along their transversal $(\tau$-)direction. Before writing down the action for the flavour branes in terms of $A_{m}$ and $\tau$, a few comments are in order.

While the low energy effective action for a single brane is known to be the DiracBorn-Infeld action [53, 54], valid in the static gauge (i.e. alignment of the world volume with space-time coordinates) and for slowly varying field strengths, the full non-Abelian generalization of it for the description of a stack of coinciding branes is not. Tseytlin proposed in [55] to non-Abelianize the Dirac-Born-Infeld action by introducing a symmetrized trace STr. The action is still restricted to static gauge and the (in the non-Abelian case slightly ambiguous) slowly-varying field strengths approximation, ignoring derivative terms including $[F, F] \sim[D, D] F$ terms. This action was shown to be valid up to fourth order in the field strength, with deviations starting to appear at order $F^{6}[59,60]$. For the probe flavour branes we are dealing with, it is given by the following, which we will further refer to as 'the' (non-Abelian) DBI-action [55-58]:

$$
S_{D B I}=-T_{8} \int d^{4} x 2 \int_{u_{0}}^{\infty} d u \int \epsilon_{4} e^{-\phi} \mathrm{S} \operatorname{Tr} \sqrt{-\operatorname{det}\left[g_{m n}^{D 8}+\left(2 \pi \alpha^{\prime}\right) i F_{m n}\right]}
$$

where $T_{8}=1 /\left((2 \pi)^{8} \ell_{s}^{9}\right)$ is the D8-brane tension, the factor 2 in front of the $u$-integration makes sure that we integrate over both halves of the $\cup$-shaped D8-branes, STr is the symmetrized trace which is defined as

$$
\operatorname{STr}\left(F_{1} \cdots F_{n}\right)=\frac{1}{n !} \operatorname{Tr}\left(F_{1} \cdots F_{n}+\text { all permutations }\right),
$$

$g_{m n}^{D 8}$ is the induced metric on the D8-branes,

$$
g_{m n}^{D 8}=g_{m n}+g_{\tau \tau}\left(D_{m} \tau\right)\left(D_{n} \tau\right),
$$

with covariant derivative $D_{m} \tau=\partial_{m} \tau+\left[A_{m}, \tau\right]$, and

$$
F_{m n}=\partial_{m} A_{n}-\partial_{n} A_{m}+\left[A_{m}, A_{n}\right]=F_{m n}^{a} t^{a}
$$

the field strength with anti-Hermitian generators

$$
t^{a}=-\frac{i}{2}\left(\mathbb{1}, \sigma_{1}, \sigma_{2}, \sigma_{3}\right), \quad \operatorname{Tr}\left(t^{a} t^{b}\right)=-\frac{\delta_{a b}}{2}, \quad\left[t^{a}, t^{b}\right]=\epsilon_{a b c} t^{c} .
$$

\subsection{Effect of uniform magnetic field on the probe branes' embedding}

To model a uniform magnetic field $\vec{B}=B \vec{e}_{3}$ in the dual field theory, $B=F_{12}^{e m}=\partial_{1} A_{2}^{e m}$, we assume the background gauge field ansatz ( $e$ being the electromagnetic coupling constant 
and $Q_{e m}$ the electric charge matrix) [3]

$$
\begin{aligned}
A_{\mu}=\bar{A}_{\mu} & =-i e Q_{e m} A_{\mu}^{e m} \quad \text { (all other gauge field components zero) } \\
& =-i x_{1}\left(\begin{array}{cc}
\frac{2}{3} e B & 0 \\
0 & -\frac{1}{3} e B
\end{array}\right) \delta_{\mu 2}=\frac{x_{1} e B \delta_{\mu 2}}{3}\left(-\frac{i \mathbb{1}_{2}}{2}\right)+x_{1} e B \delta_{\mu 2}\left(-\frac{i \sigma_{3}}{2}\right),
\end{aligned}
$$

or

$$
\bar{A}_{2}^{3}=x_{1} e B \quad \text { and } \quad \bar{A}_{2}^{0}=\bar{A}_{2}^{3} / 3
$$

and

$$
\bar{F}_{12}=\partial_{1} \bar{A}_{2}=-i\left(\begin{array}{cc}
\frac{2}{3} e B & 0 \\
0 & -\frac{1}{3} e B
\end{array}\right)=-i\left(\begin{array}{cc}
\bar{F}_{u} & 0 \\
0 & \bar{F}_{d}
\end{array}\right),
$$

where in the last line we defined the up- and down-components of the background field strength, $\bar{F}_{u}$ and $\bar{F}_{d}$. In the rest of the paper we will denote $e B$ as $B$.

The embedding of the $(8+1)$-dimensional D8-branes in the 10-dimensional D4-brane background (3.1) only requires the specification of one function, $\tau(u)$. This embedding function can be determined as a function of $B$ by first plugging the above gauge field ansatz into the DBI-action (3.10), together with the metric ansatz

$$
g^{D 8}=\left(\begin{array}{cc}
g_{u}^{D 8} & 0 \\
0 & g_{d}^{D 8}
\end{array}\right)
$$

to allow for a different response of up- and down-brane to the magnetic field. Subsequently one can solve for $u^{\prime}=d u / d \tau$ (for each flavour) by expressing conservation $\partial_{\tau} H=0$ of the 'Hamiltonian' $H=u^{\prime} \frac{\delta \mathcal{L}^{\tau}}{\delta u^{\prime}}-\mathcal{L}^{\tau}$ with $S_{D B I} \sim \int d \tau \mathcal{L}^{\tau}$ and assuming a $\cup$-shaped embedding, i.e. $u^{\prime}=0$ at $u=u_{0}$. The result for the $B$-dependent embedding is (for more details, see [1]):

$$
\tau(u)=\bar{\tau}=\left(\begin{array}{cc}
\bar{\tau}_{u} & 0 \\
0 & \bar{\tau}_{d}
\end{array}\right)
$$

with

$$
\partial_{u} \bar{\tau}_{l}=\sqrt{\left(\frac{R}{u}\right)^{3} \frac{1}{f^{2}} \frac{u_{0, l}^{8} f_{0, l} A_{0, l}}{u^{8} f A_{l}-u_{0, l}^{8} f_{0, l} A_{0, l}}} \times \theta\left(u-u_{0, l}\right), \quad(l=u, d)
$$

where $f$ is short for $f(u)=1-u_{K}^{3} / u^{3}, A_{0}$ and $f_{0}$ stand for $A\left(u_{0}\right)$ and $f\left(u_{0}\right), \theta\left(u-u_{0, l}\right)$ is the Heaviside stepfunction, and all the $B$-dependence is collected in the newly defined matrix $A$ :

$$
A=\left(\begin{array}{cc}
A_{u} & 0 \\
0 & A_{d}
\end{array}\right)=1-\left(2 \pi \alpha^{\prime}\right)^{2} \bar{F}_{12}^{2}\left(\frac{R}{u}\right)^{3}, \quad A_{l}=1+\left(2 \pi \alpha^{\prime}\right)^{2} \bar{F}_{l}^{2}\left(\frac{R}{u}\right)^{3}, \quad(l=u, d) .
$$

The up- and down-brane are thus no longer coincident in the presence of $B$, as sketched in figure 2.

The splitting of the branes represents the magnetically induced explicit breaking of global chiral symmetry,

$$
\mathrm{U}(2)_{L} \times \mathrm{U}(2)_{R} \stackrel{B}{\rightarrow}\left(\mathrm{U}(1)_{L} \times \mathrm{U}(1)_{R}\right)^{u} \times\left(\mathrm{U}(1)_{L} \times \mathrm{U}(1)_{R}\right)^{d},
$$



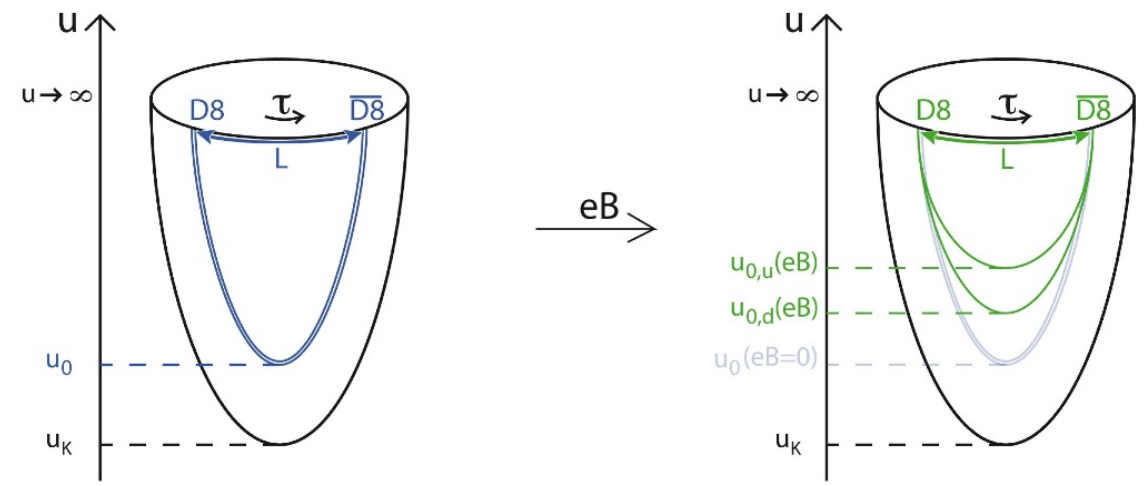

Figure 2. The change in embedding of the flavour branes caused by the magnetic field $B$ models the chiral magnetic catalysis effect. The up-brane reacts the strongest to $B$, corresponding to a stronger chiral magnetic catalysis for the up-quarks than for the down-quarks.

caused by the up- and down-quarks' different coupling to the magnetic field. This is also reflected in the fact that the non-Abelian DBI-action for the two D8-branes reduces to the sum of two Abelian actions (the STr reduces to an ordinary Tr because the embedding matrix (3.17) is diagonal).

The $B$-dependence of $u_{0, u}$ and $u_{0, d}$ is determined by keeping the asymptotic separation $L$ between D8- and $\overline{\mathrm{D} 8}$-branes, as a function of $B$ given by

$$
L=2 \int_{u_{0}}^{\infty} d u\left(\frac{R}{u}\right)^{3 / 2} f^{-1} \sqrt{\frac{u_{0}^{8} f_{0} A_{0}}{u^{8} f A-u_{0}^{8} f_{0} A_{0}}},
$$

fixed to its value at $B=0 . L$ serves as the boundary condition on the branes' embedding, ${ }^{2}$ see also for example the work of Preis et al. [16-19]. The $B$-dependence of the constituent quark masses then follows directly from (3.7), or in terms of the fixed parameters

$$
m_{q}\left(M_{K}, u_{0}, \kappa\right)=8 \pi^{2} M_{K}^{2} \kappa \int_{1 / M_{K}}^{u_{0}} d u \frac{1}{\sqrt{1-\frac{1}{\left(M_{K} u\right)^{3}}}} .
$$

The results for $u_{0}(B)$ and $m_{q}(B)$ (for both flavours) are shown in figure 3 . The rising of the constituent masses $m_{q}$ with $B$ is consistent with the interpretation of the $B$-dependent embedding as a modeling of the chiral magnetic catalysis effect (as already discussed in the Sakai-Sugimoto model in [16-19]): as the value of $u_{0}$, where the branes merge, rises, the $\cup$-shaped embedding gets more strongly bent, diverging more and more from the chirally invariant embedding of straight branes. The up-brane reacts twice as strongly to the presence of $e B$, corresponding to a stronger chiral magnetic catalysis for the up-quarks than for

\footnotetext{
${ }^{2}$ From the perspective of the asymptotic dual field theory, the flavour branes are infinitely extended, massive objects in the bulk, requiring an infinite amount of energy to move them. In this sense it is natural to keep $L$ fixed as a boundary condition to probe the effects of the bulk dynamics in the presence of the external field. The value of $L$ determines how much of the gluonic bulk dynamics is probed, ranging from all $\left(u_{0}=u_{K}\right)$ for maximal $L$ to none $\left(u_{0} \rightarrow \infty\right)$ for minimal $L$. In this interpretation, the choice of $L$ (which has no direct physical meaning in the dual field theory) corresponds to the choice of type of dual field theory, ranging from QCD-like to NJL-like in the limit of $L \rightarrow 0$ or $\tau$ non-compact. To avoid confusion, with "NJLlike" we refer to a model sharing some but not all features with NJL-models. For more details, see [61].
} 

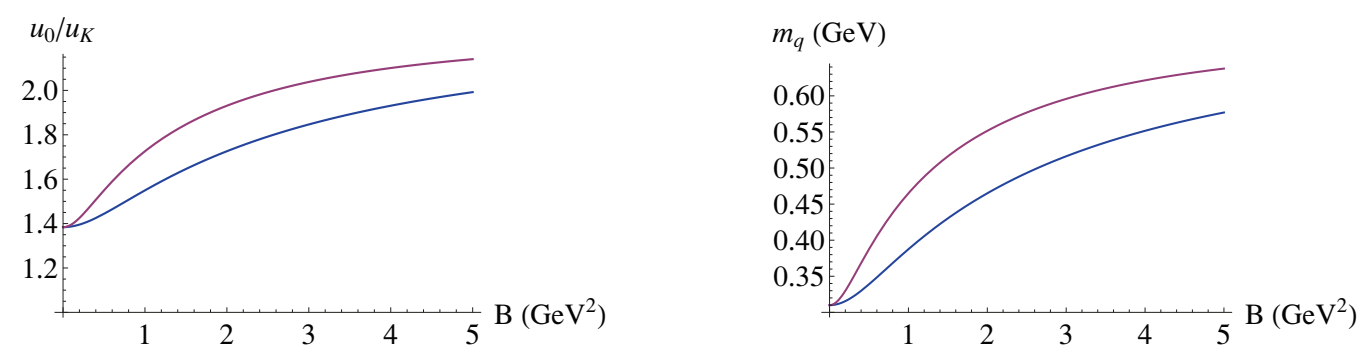

Figure 3. (a) $\frac{u_{0}}{u_{K}}$ as a function of the magnetic field for the D8-brane corresponding to the upquark (red), and the one corresponding to the down-quark (blue). (b) The constituent masses of the up-quark (red) and the down-quark (blue) as a function of the magnetic field.

the down-quarks. In the special case of an antipodal embedding $u_{0}=u_{K}$ at $B=0$, turning on the magnetic field has no influence on the brane embedding: $f_{0}=0 \Rightarrow \partial \bar{\tau}=0 \Rightarrow \bar{\tau} \sim \mathbb{1}$ so the branes remain antipodal and coincident for all values of the applied magnetic field. Hence choosing the extremal antipodal model corresponds to ignoring the magnetic catalysis.

It is interesting to notice that a similarly shaped plot as in figure $3 \mathrm{~b}$ was presented in [62, figure 12] for the in [48] proposed order parameter in terms of a background magnetic field (be it for the case of a non-compact $\tau$-direction).

\section{Stability analysis}

To investigate the stability of the set-up with respect to gauge and scalar field fluctuations, let us first derive the form of the action to second order in the fluctuations by plugging the total gauge field ansatz

$$
\left\{\begin{array}{l}
A_{r}=\bar{A}_{r}+\tilde{A}_{r} \quad(r=\mu, u) \\
\tau=\bar{\tau}+\tilde{\tau}
\end{array}\right.
$$

with (see (3.13) and (3.18))

$$
\left\{\begin{array}{l}
\bar{A}_{\mu}=-i e Q_{e m} x_{1} B \delta_{\mu 2} \\
\partial_{u} \bar{\tau}=\sqrt{\left(\frac{R}{u}\right)^{3} \frac{1}{f^{2}} \frac{u_{0}^{8} f_{0} A_{0}}{u^{8} f A-u_{0}^{8} f_{0} A_{0}}} \times \theta\left(u-u_{0}\right),
\end{array}\right.
$$

into the DBI-action (3.10). The background components of the field ansatz (4.1) describe the background magnetic field (in $\bar{A}_{\mu}$ ) and the (B-dependent) embedding of the branes (in $\left.\partial_{u} \bar{\tau}\right)$. The fluctuation components will be related to resp. vector and scalar mesons in the dual field theory.

We have to evaluate (for notational brevity we temporarily absorb the factor $\left(2 \pi \alpha^{\prime}\right)$ into the field strength)

$$
2 \int d u \operatorname{STr} \sqrt{-\operatorname{det}\left(a_{r s}\right)}=2 \int d u \operatorname{STr} \sqrt{-\operatorname{det}\left(g_{r s}^{D 8}+i F_{r s}\right)},
$$

with

$$
g_{r s}^{D 8}=g_{r s}+g_{\tau \tau} D_{r} \tau D_{s} \tau, \quad \text { with } D_{r} \cdot=\partial_{r}+\left[\bar{A}_{r}, \cdot\right]
$$


and

$$
F_{r s}=\partial_{r} A_{s}-\partial_{s} A_{r}+\left[A_{r}, A_{s}\right]
$$

If the argument $a$ of the determinant (which runs over the Lorentz-indices) is written as

$$
a=\bar{a}+a^{(1)}+a^{(2)}+\cdots
$$

with $a^{(n)}$ being $n$-th order in the fluctuations $\tilde{A}$, the determinant can be expanded to second order in the fluctuations as follows

$$
\left.\sqrt{-\operatorname{det} a}\right|_{\tilde{A}^{2}}=\sqrt{-\operatorname{det} \bar{a}}\left\{1+\frac{1}{2} \operatorname{tr}\left(\bar{a}^{-1} a^{(1)}\right)+\frac{1}{8}\left(\operatorname{tr}\left(\bar{a}^{-1} a^{(1)}\right)\right)^{2}-\frac{1}{4} \operatorname{tr}\left(\left(\bar{a}^{-1} a^{(1)}\right)^{2}\right)+\frac{1}{2} \operatorname{tr}\left(\bar{a}^{-1} a^{(2)}\right)\right\} .
$$

We denote the trace in Lorentz-space with a small tr, and the trace in flavour space with a capital (S)Tr. Splitting each component of $a$ in its symmetric and antisymmetric parts

$$
\left\{\begin{array}{l}
\bar{a}^{-1}=\mathcal{G}+\mathcal{B} \\
a^{(1)}=\bar{a}^{(1)}+\delta_{1} F \\
a^{(2)}=\bar{a}^{(2)}+\delta_{2} F
\end{array}\right.
$$

the expansion of the determinant (4.6) to second order in the fluctuations becomes

$$
\begin{aligned}
\sqrt{-\operatorname{det} a} & \tilde{A}^{2}=\sqrt{-\operatorname{det} \bar{a}}+\sqrt{-\operatorname{det} \bar{a}} \times \\
\times & \left\{\frac{1}{2} \operatorname{tr}\left(\mathcal{G} \bar{a}^{(1)}\right)+\frac{1}{8}\left(\operatorname{tr}\left(\mathcal{G} \bar{a}^{(1)}\right)\right)^{2}-\frac{1}{4} \operatorname{tr}\left(\mathcal{G} \bar{a}^{(1)} \mathcal{G} \bar{a}^{(1)}+\mathcal{B} \bar{a}^{(1)} \mathcal{B} \bar{a}^{(1)}\right)+\frac{1}{2} \operatorname{tr}\left(\mathcal{G} \bar{a}^{(2)}\right)\right. \\
& +\frac{1}{2} \operatorname{tr}\left(\mathcal{B} \delta_{1} F\right)+\frac{1}{8}\left(\operatorname{tr}\left(\mathcal{B} \delta_{1} F\right)\right)^{2}-\frac{1}{4} \operatorname{tr}\left(\mathcal{G} \delta_{1} F \mathcal{G} \delta_{1} F+\mathcal{B} \delta_{1} F \mathcal{B} \delta_{1} F\right)+\frac{1}{2} \operatorname{tr}\left(\mathcal{B} \delta_{2} F\right) \\
& \left.+\frac{1}{4} \operatorname{tr}\left(\mathcal{G} \bar{a}^{(1)}\right) \operatorname{tr}\left(\mathcal{B} \delta_{1} F\right)-\frac{1}{2} \operatorname{tr}\left(\mathcal{G} \bar{a}^{(1)} \mathcal{B} \delta_{1} F\right)-\frac{1}{2} \operatorname{tr}\left(\mathcal{G} \delta_{1} F \mathcal{B} \bar{a}^{(1)}\right)\right\}
\end{aligned}
$$

For our field ansatz we have

$$
\begin{aligned}
\bar{a}_{r s} & =g_{r s}+g_{\tau \tau} \partial_{r} \bar{\tau} \partial_{s} \bar{\tau}+i \bar{F}_{r s} \\
\bar{a}_{r s}^{(1)} & =g_{\tau \tau}\left(\partial_{r} \bar{\tau}\left(\left[\tilde{A}_{s}, \bar{\tau}\right]+D_{s} \tilde{\tau}\right)+\left(\left[\tilde{A}_{r}, \bar{\tau}\right]+D_{r} \tilde{\tau}\right) \partial_{s} \bar{\tau}\right) \\
\delta_{1} F_{r s} & =i\left(D_{r} \tilde{A}_{s}-D_{s} \tilde{A}_{r}\right) \stackrel{\text { notation }}{=} i \tilde{F}_{r s} \\
\bar{a}_{r s}^{(2)} & =g_{\tau \tau}\left(\left[\tilde{A}_{r}, \bar{\tau}\right]+D_{r} \tilde{\tau}\right)\left(\left[\tilde{A}_{s}, \bar{\tau}\right]+D_{s} \tilde{\tau}\right)+g_{\tau \tau}\left(\left[\tilde{A}_{r}, \tilde{\tau}\right] \partial_{s} \bar{\tau}+\partial_{r} \bar{\tau}\left[\tilde{A}_{r}, \tilde{\tau}\right]\right), \\
\delta_{2} F_{r s} & =i\left[\tilde{A}_{r}, \tilde{A}_{s}\right] .
\end{aligned}
$$

The symmetric part $\mathcal{G}$ of $\bar{a}^{-1}$ is diagonal,

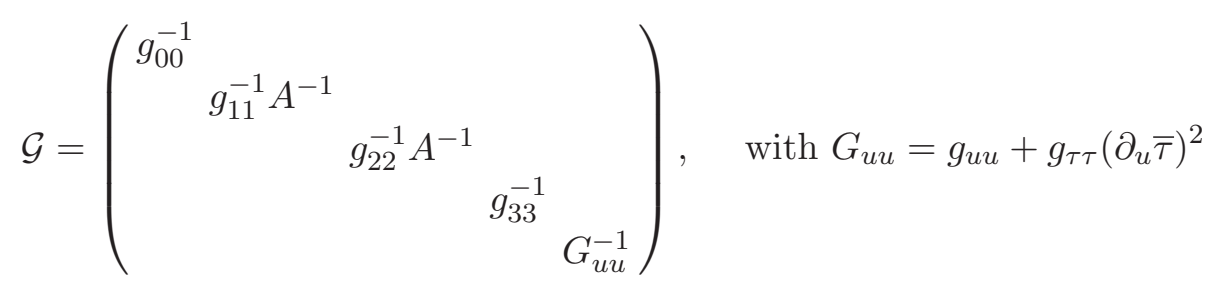


and the antisymmetric part $\mathcal{B}$ has non-zero components

$$
\mathcal{B}_{12}=-\mathcal{B}_{21}=i \bar{F}_{12} g_{11}^{-1} g_{22}^{-1} A^{-1}
$$

As a check, the first order terms in (4.8) do vanish on-shell, that is upon using the embedding function (3.18). The DBI-Lagrangian to second order in the fluctuations then reads

$$
\left.\operatorname{STr} e^{-\phi} \sqrt{-\operatorname{det} a}\right|_{\tilde{A}^{2}, \tilde{\tau}^{2}, \tilde{A} \tilde{\tau}}=\mathcal{L}_{1}+\mathcal{L}_{2}+\mathcal{L}_{3}+\mathcal{L}_{4}
$$

with

$$
\begin{aligned}
& \mathcal{L}_{1}=\operatorname{Tr} e^{-\phi} \sqrt{-\operatorname{det} \bar{a}} \\
& \mathcal{L}_{2}=\operatorname{STr} \bar{x}\left\{\frac{1}{2}\left(\left[\tilde{A}_{u}, \bar{\tau}\right]+D_{u} \tilde{\tau}\right)^{2} G_{u u}^{-2}+\bar{y}\left[\tilde{A}_{u}, \tilde{\tau}\right]+\left.\frac{1}{2}\left(\left[\tilde{A}_{\mu}, \bar{\tau}\right]+D_{\mu} \tilde{\tau}\right)^{2} g_{\mu \mu}^{-1} A^{-1}\right|_{\mu=1,2} G_{u u}^{-1}\right\} \\
& \mathcal{L}_{3}=\operatorname{STr} \bar{x}\left\{-\bar{F}_{12} g_{11}^{-1} g_{22}^{-1} A^{-1}\left[\tilde{A}_{1}, \tilde{A}_{2}\right]-\left.\frac{1}{4} g_{\mu \mu}^{-1} g_{\nu \nu}^{-1} A^{-2}\right|_{\mu, \nu=1,2} \tilde{F}_{\mu \nu}^{2}-\left.\frac{1}{2} g_{\mu \mu}^{-1} A^{-1}\right|_{\mu=1,2} G_{u u}^{-1} \tilde{F}_{\mu u}^{2}\right\} \\
& \mathcal{L}_{4}=\operatorname{STr} \bar{x}\left\{-\bar{z}\left(\left(\left[\tilde{A}_{u}, \bar{\tau}\right]+D_{u} \tilde{\tau}\right) \tilde{F}_{12}+\left(\left[\tilde{A}_{1}, \bar{\tau}\right]+D_{1} \tilde{\tau}\right) \tilde{F}_{2 u}-\left(\left[\tilde{A}_{2}, \bar{\tau}\right]+D_{2} \tilde{\tau}\right) \tilde{F}_{1 u}\right)\right\},
\end{aligned}
$$

where

$$
\bar{x}=e^{-\phi} \sqrt{-\operatorname{det} \bar{a}}=e^{-\phi} g_{11}^{2} \sqrt{G_{u u}} g_{S_{4}}^{2} \sqrt{A}, \quad \bar{y}=G_{u u}^{-1} g_{\tau \tau} \partial_{u} \bar{\tau}, \quad \bar{z}=\bar{y} \bar{F}_{12} g_{11}^{-1} g_{22}^{-1} A^{-1}
$$

are functions of the background fields $\partial_{u} \bar{\tau}$ and $\bar{F}_{12}$, so functions of $u$ only, and diagonal in flavour space. The notation for the factors $\left.g_{\mu \mu}^{-1} A^{-1}\right|_{\mu=1,2}$ coming from $\mathcal{G}$ means that $g_{\mu \mu}^{-1}$ is accompanied with a factor $A^{-1}=\frac{1}{1-\left(2 \pi \alpha^{\prime}\right)^{2} \bar{F}_{12}^{2} R^{3} / u^{3}}$ only for $\mu=1,2$.

\subsection{Gauge fixing}

\subsubsection{STr-evaluation}

The action (4.17) contains mixing terms between the scalar and gauge fluctuations in $\mathcal{L}_{2}$ and $\mathcal{L}_{4}$. We will disentangle these couplings here by choosing a particular gauge. First we work out $\mathcal{L}_{2}$ a bit further by evaluating the $\mathrm{S} \operatorname{Tr}$ (3.11). According to its definition in [56] the STr takes a symmetric average over all orderings of $F_{m n}, D_{m} \tau$ and $\tau$ appearing in the non-Abelian Taylor expansions of the fields in the action. In particular, commutators, such as $\left[A_{m}, A_{n}\right]$ in $F_{m n}$ or $\left[A_{m}, \tau\right]$ in $D_{m} \tau$, are handled as one matrix. The STr-expressions we encounter in (4.17) can be classified into two types: expressions of the form $\operatorname{STr}\left(\mathcal{H}\left(\partial_{u} \bar{\tau}\right) \mathcal{G}\left(\bar{F}_{12}\right) \tilde{X}\right)$ and $\operatorname{STr}\left(\mathcal{H}\left(\partial_{u} \bar{\tau}\right) \mathcal{G}\left(\bar{F}_{12}\right) \tilde{X}^{2}\right)$. Here $\mathcal{H}$, resp. $\mathcal{G}$ are even functions of the diagonal background field

$$
\bar{\tau}=\bar{\tau}^{0} \sigma^{0}+\bar{\tau}^{3} \sigma^{3},
$$

resp.

$$
\bar{F}_{12}=F^{0} \sigma^{0}+F^{3} \sigma^{3}=-\frac{i}{2} \frac{B}{3} \sigma^{0}-\frac{i}{2} B \sigma^{3},
$$


and $\tilde{X}=\tilde{X}^{a} t^{a}$ is some fluctuation - in the present case fully general fluctuations $D_{m} \tilde{\tau}$ and off-diagonal fluctuations $\left[\tilde{A}_{m}, \bar{\tau}\right]$. For expressions of these types the STr can be evaluated exactly $[59,63]$ as elaborated on in the appendix A. Using the prescriptions presented and rederived there, we arrive at the following form for $\mathcal{L}_{2}$ :

$$
\begin{aligned}
\mathcal{L}_{2}= & \sum_{a=1}^{2}\left\{\gamma(u) \frac{1}{2}\left(\left[\tilde{A}_{u}, \bar{\tau}\right]^{a}+\partial_{u} \tilde{\tau}^{a}\right)^{2}+\alpha(u) \frac{1}{2}\left(\left[\tilde{A}_{\mu}, \bar{\tau}\right]^{a}+D_{\mu} \tilde{\tau}^{a}\right)^{2}+\beta(u) \sum_{\mu=1}^{2} \frac{1}{2}\left(\left[\tilde{A}_{\mu}, \bar{\tau}\right]^{a}+D_{\mu} \tilde{\tau}^{a}\right)^{2}\right\} \\
& +\operatorname{Tr}\left(\overline{x y}\left[\tilde{A}_{u}, \tilde{\tau}\right]\right)+\sum_{l=u, d}\left\{\gamma_{l}(u) \frac{1}{2}\left(\partial_{u} \tilde{\tau}^{l}\right)^{2}+\alpha_{l}(u) \frac{1}{2}\left(D_{\mu} \tilde{\tau}^{l}\right)^{2}+\beta_{l}(u) \sum_{\mu=1}^{2} \frac{1}{2}\left(D_{\mu} \tilde{\tau}^{l}\right)^{2}\right\}
\end{aligned}
$$

with

$$
\begin{aligned}
& \gamma(u)=-\frac{1}{2} I\left(\bar{x} G_{u u}^{-2}\right), \quad \alpha(u)=-\frac{1}{2} I\left(\bar{x} g_{11}^{-1} G_{u u}^{-1}\right), \quad \beta(u)=-\frac{1}{2} I\left(\bar{x} g_{11}^{-1} G_{u u}^{-1} \frac{1-A}{A}\right), \\
& \gamma_{l}(u)=-\frac{1}{2} I_{l}\left(\bar{x} G_{u u}^{-2}\right), \quad \alpha_{l}(u)=-\frac{1}{2} I_{l}\left(\bar{x} g_{11}^{-1} G_{u u}^{-1}\right), \quad \beta_{l}(u)=-\frac{1}{2} I_{l}\left(\bar{x} g_{11}^{-1} G_{u u}^{-1} \frac{1-A}{A}\right)
\end{aligned}
$$

containing what we will refer to as ' $I$-functions' and ' $I_{l}$-functions', defined in (A.7) and (A.8), e.g.

$$
\begin{aligned}
I\left(\bar{x} G_{u u}^{-2}\right) & =e^{-\phi} g_{11}^{2} g_{S_{4}}^{2} I\left(G_{u u}^{-3 / 2}(\partial \bar{\tau}) A^{1 / 2}\left(\bar{F}_{12}\right)\right) \\
& =\frac{e^{-\phi} g_{11}^{2} g_{S_{4}}^{2}}{2} \int_{0}^{1} d \alpha\left\{G_{u u}^{-3 / 2}\left(\partial \bar{\tau}^{0}+\alpha \partial \bar{\tau}^{3}\right) A^{1 / 2}\left(F^{0}+\alpha F^{3}\right)+G_{u u}^{-3 / 2}\left(\partial \bar{\tau}^{0}-\alpha \partial \bar{\tau}^{3}\right) A^{1 / 2}\left(F^{0}-\alpha F^{3}\right)\right\}, \\
I_{u}\left(\bar{x} G_{u u}^{-2}\right) & =e^{-\phi} g_{11}^{2} g_{S_{4}}^{2} G_{u u}^{-3 / 2}\left(\partial \bar{\tau}^{0}+\partial \bar{\tau}^{3}\right) A^{1 / 2}\left(F^{0}+F^{3}\right),
\end{aligned}
$$

with $\partial \bar{\tau}$ short for $\partial_{u} \bar{\tau}$ and (with $\tilde{\tau}=\tilde{\tau}^{a} t^{a}$ )

$$
\tilde{\tau}^{l}=\frac{\tilde{\tau}^{0} \pm \tilde{\tau}^{3}}{\sqrt{2}}
$$

Having used $g_{\mu \nu}=g_{11} \eta_{\mu \nu}$ and absorbing $\eta_{\mu \nu}$ in the notation of the squares, $\left(\partial_{\mu} \tilde{\tau}^{a}\right)^{2}=$ $\partial_{\mu} \tilde{\tau}^{a} \partial_{\nu} \tilde{\tau}^{a} \eta^{\mu \nu}=\partial_{\mu} \tilde{\tau}^{a} \partial^{\mu} \tilde{\tau}^{a}$, all the products over $\mu$ in the above Lagrangian (and in all expressions following unless stated otherwise) are contracted Minkowski products.

The difficulty in evaluating the STr, although we restrict to second order in the fluctuations, comes from the presence of the background fields $\partial \bar{\tau}$ (appearing in the induced metric on the flavour branes through $G_{u u}=g_{u u}+g_{\tau \tau}\left(\partial_{u} \bar{\tau}\right)^{2}$ ) and $\bar{F}_{12}$ (appearing in $A$ as defined in (3.19)), which have to be ordered ${ }^{3}$ within the STr. The functions containing the background fields have to be Taylor expanded before the ordering and subsequently resummed. This gives rise to complicated $I$-functions as in (4.20), which in general have to be calculated numerically.

\subsubsection{Choosing a 't Hooft gauge}

We consider a 't Hooft gauge-fixing function [64] in the non-Abelian directions - assuming the Einstein convention that double $\mathrm{SU}(2)$-indices $b, c=1,2,3$ are summed over -

$$
G^{a}=\frac{1}{\sqrt{\xi}}\left(\alpha(u) D_{\mu} \tilde{A}_{\mu}^{a}+\gamma(u) D_{u} \tilde{A}_{u}^{a}+\sum_{i=1,2} \beta(u) D_{i} \tilde{A}_{i}^{a}\right)+2 i \sqrt{\xi} \epsilon_{a b c} \tilde{\tau}^{b} \bar{\tau}^{c} \quad(a=1,2)
$$

\footnotetext{
${ }^{3}$ There is some ambiguity here in the sense that the background scalar field $\partial_{u} \bar{\tau}$ itself depends on the background gauge field $\bar{F}_{12}$, so there is also the option to order the matrices $\bar{F}_{12}$ within $\partial_{u} \bar{\tau}$, as opposed to ordering $\partial_{u} \bar{\tau}$ as independent. We however opted for the latter, which seems more logical to us.
} 
such that the gauge-fixed Lagrangian

$$
\begin{aligned}
\mathcal{L}_{2} & -\frac{1}{2}\left(G^{a}\right)^{2}=\sum_{a=1}^{2}\left\{\gamma(u) \frac{1}{2}\left[\left(\left[\tilde{A}_{u}, \bar{\tau}\right]^{a}\right)^{2}+\left(\partial_{u} \tilde{\tau}^{a}\right)^{2}\right]+\alpha(u) \frac{1}{2}\left[\left(\left[\tilde{A}_{\mu}, \bar{\tau}\right]^{a}\right)^{2}+\left(D_{\mu} \tilde{\tau}^{a}\right)^{2}\right]\right. \\
& \left.+\beta(u) \sum_{\mu=1}^{2} \frac{1}{2}\left[\left(\left[\tilde{A}_{\mu}, \bar{\tau}\right]^{a}\right)^{2}+\left(D_{\mu} \tilde{\tau}^{a}\right)^{2}\right]-\frac{1}{2 \xi}\left[(D \tilde{A})^{2} \text { terms }\right]+\frac{1}{2}\left(\sqrt{\xi} \tilde{\tau}^{a}\right)^{2}\left(2 \bar{\tau}^{3}\right)^{2}+2 i \tilde{A}_{u}^{a} \epsilon_{a b c} \tilde{\tau}^{b} \partial_{u}\left(\gamma(u) \bar{\tau}^{c}\right)\right\} \\
& +\operatorname{Tr}\left(\overline{x y}\left[\tilde{A}_{u}, \tilde{\tau}\right]\right)+\sum_{l=u, d}\{\cdots\}
\end{aligned}
$$

will be free of mixing terms for a sensible choice of the gauge parameter $\xi$. The Lagrangian $\mathcal{L}$ is replaced by $\mathcal{L}-\frac{1}{2}\left(G^{a}\right)^{2}$ by virtue of the Faddeev-Popov trick: the partition function of a system with action $S=\int d x \mathcal{L}$ fulfilling the gauge-fixing constraints $G^{a}(A, \tau)=0$ is written as

$$
Z=\int \mathcal{D} A \mathcal{D} \tau e^{i \int d x \mathcal{L}(A, \tau)} \sim \int \mathcal{D} A \mathcal{D} \tau e^{i \int d x \mathcal{L}(A, \tau)} \delta[G(A, \tau)] \Delta_{G(A, \tau)}
$$

with proportionality constant the volume of the gauge group, $\delta[G(A, \tau)]=$ $\Pi_{x, a}\left(\delta\left[G^{a}(A(x), \tau(x))\right]\right)$ and $\Delta_{G(A, \tau)}$ the associated Jacobian, or alternatively through introducing the gauge-fixing as $\delta\left(G^{a}(A(x), \tau(x))-\omega^{a}(x)\right)$ and integrating over $\omega^{a}$ having a Gaussian distribution around zero - as

$$
Z \sim \int \mathcal{D} A \mathcal{D} \tau e^{i \int d x\left[\mathcal{L}(A, \tau)-\frac{1}{2}\left(G^{a}(A, \tau)\right)^{2}\right]} \Delta_{G(A, \tau)}
$$

Now we rescale the charged scalar fluctuations $\tilde{\tau}^{a=1,2} \rightarrow \frac{\tilde{\tau}^{a=1,2}}{\sqrt{\xi}}$ and choose the so-called 'unitary' gauge

$$
\xi \rightarrow \infty \text {. }
$$

This boils down to deleting all dynamical terms for the fluctuations $\tilde{\tau}^{a=1,2}$ and we are left with

$$
\begin{aligned}
\mathcal{L}_{2}-\frac{1}{2}\left(G^{a}\right)^{2}= & \sum_{a=1}^{2}\left\{\gamma(u) \frac{1}{2}\left(\left[\tilde{A}_{u}, \bar{\tau}\right]^{a}\right)^{2}+\alpha(u) \frac{1}{2}\left(\left[\tilde{A}_{\mu}, \bar{\tau}\right]^{a}\right)^{2}+\beta(u) \sum_{\mu=1}^{2} \frac{1}{2}\left(\left[\tilde{A}_{\mu}, \bar{\tau}\right]^{a}\right)^{2}+\frac{1}{2}\left(\tilde{\tau}^{a}\right)^{2}\left(2 \bar{\tau}^{3}\right)^{2}\right\} \\
& +\sum_{l=u, d}\{\cdots\} .
\end{aligned}
$$

With the above gauge choice we can see the Higgs mechanism at work that is associated with the magnetic field pulling the up- and down-brane apart: the charged scalar fluctuations $\tilde{\tau}^{1,2}$ now serve as Goldstone bosons that are eaten by the gauge bosons $\tilde{A}_{m}^{1,2}$, acquiring a mass $\sim\left(\bar{\tau}^{3}\right)^{2}$, where $\bar{\tau}^{3}$ is essentially the vacuum expectation value of the diagonal component $\tau^{3}$ of the $\tau$-field. The remaining fluctuations $\tilde{\tau}^{0,3}$ are the Higgs bosons.

\subsubsection{Fixing the remaining gauge freedom}

In the unitary gauge, $\mathcal{L}_{4}$, containing the only remaining mixing terms between gauge and scalar fluctuations, reads

$$
\begin{aligned}
\mathcal{L}_{4} & =\frac{1}{2}\left\{I(\overline{x z}) \sum_{a=1}^{2}\left[\left[\tilde{A}_{u}, \bar{\tau}\right]^{a} \tilde{F}_{12}^{a}+\left[\tilde{A}_{1}, \bar{\tau}\right]^{a} \tilde{F}_{2 u}^{a}-\left[\tilde{A}_{2}, \bar{\tau}\right]^{a} \tilde{F}_{1 u}^{a}\right]+\sum_{l=u, d} I_{l}(\overline{x z})\left[D_{u} \tilde{\tau}^{l} \tilde{F}_{12}^{l}+D_{1} \tilde{\tau}^{l} \tilde{F}_{2 u}^{l}-D_{2} \tilde{\tau}^{l} \tilde{F}_{1 u}^{l}\right]\right\} \\
& =\frac{1}{2} I(\overline{x z}) \sum_{a=1}^{2}\left(-\left[\tilde{A}_{1}, \bar{\tau}\right]^{a} \partial_{u} \tilde{A}_{2}^{a}+\left[\tilde{A}_{2}, \bar{\tau}\right]^{a} \partial_{u} \tilde{A}_{1}^{a}\right)
\end{aligned}
$$


where we used partial integration. The neutral part vanishes due to the gauge choice

$$
A_{u}^{3}=A_{u}^{0}=0
$$

hereby using the remaining gauge freedom in the $a=0,3$ directions, as the 't Hooft gauge (4.22) only fixes the gauge for $a=1,2$.

In the chosen gauge (4.22), (4.25), (4.28), the Lagrangian is free of $\tilde{A}_{m} \tilde{\tau}$ couplings:

$$
\left.\operatorname{STr} e^{-\phi} \sqrt{-\operatorname{det} a}\right|_{\tilde{A}^{2}, \tilde{\tau}^{2}}=\overline{\mathcal{L}}+\mathcal{L}_{\text {Higgs }}+\mathcal{L}_{\text {scalar }}+\mathcal{L}_{\text {vector }}+\mathcal{L}_{\text {vector }- \text { mixing }}
$$

with

$$
\begin{aligned}
\overline{\mathcal{L}} & =\operatorname{Tr} e^{-\phi} \sqrt{-\operatorname{det} \bar{a}} \\
\mathcal{L}_{\text {Higgs }} & =\sum_{a=1}^{2}\left\{\gamma(u) \frac{1}{2}\left(\left[\tilde{A}_{u}, \bar{\tau}\right]^{a}\right)^{2}+\alpha(u) \frac{1}{2}\left(\left[\tilde{A}_{\mu}, \bar{\tau}\right]^{a}\right)^{2}+\beta(u) \sum_{\mu=1}^{2} \frac{1}{2}\left(\left[\tilde{A}_{\mu}, \bar{\tau}\right]^{a}\right)^{2}-\frac{1}{2}\left(\tilde{\tau}^{a}\right)^{2}\left(\bar{\tau}^{3}\right)^{2}\right\} \\
\mathcal{L}_{\text {scalar }} & =\sum_{l=u, d}\left\{\gamma_{l}(u) \frac{1}{2}\left(\partial_{u} \tilde{\tau}^{l}\right)^{2}+\alpha_{l}(u) \frac{1}{2}\left(D_{\mu} \tilde{\tau}^{l}\right)^{2}+\beta_{l}(u) \sum_{\mu=1}^{2} \frac{1}{2}\left(D_{\mu} \tilde{\tau}^{l}\right)^{2}\right\} \\
\mathcal{L}_{\text {vector }} & =\operatorname{STr} \bar{x}\left\{-\bar{F}_{12} g_{11}^{-2} A^{-1}\left[\tilde{A}_{1}, \tilde{A}_{2}\right]-\left.\frac{1}{4} g_{11}^{-2} \tilde{F}_{\mu \nu}^{2} A^{-2}\right|_{\mu, \nu=1,2}-\left.\frac{1}{2} g_{11}^{-1} G_{u u}^{-1} \tilde{F}_{\mu u}^{2} A^{-1}\right|_{\mu=1,2}\right\} \\
\mathcal{L}_{\text {vector-mixing }} & =\frac{1}{2}\left\{I(\overline{x z}) \sum_{a=1}^{2}\left(-\left[\tilde{A}_{1}, \bar{\tau}\right]^{a} \partial_{u} \tilde{A}_{2}^{a}+\left[\tilde{A}_{2}, \bar{\tau}\right]^{a} \partial_{u} \tilde{A}_{1}^{a}\right)\right\}
\end{aligned}
$$

\subsection{Stability in scalar sector}

In this section we discuss the scalar part of the DBI-Lagrangian (4.30),

$$
\begin{aligned}
\mathcal{L}_{\text {scalar }} & =\left.\operatorname{STr} e^{-\phi} \sqrt{-\operatorname{det} a}\right|_{\tilde{\tau}^{2}} \\
& =\sum_{l=u, d}\left\{\gamma_{l}(u) \frac{1}{2}\left(\partial_{u} \tilde{\tau}^{l}\right)^{2}+\alpha_{l}(u) \frac{1}{2}\left(D_{\mu} \tilde{\tau}^{l}\right)^{2}+\beta_{l}(u) \sum_{\mu=1}^{2} \frac{1}{2}\left(D_{\mu} \tilde{\tau}^{l}\right)^{2}\right\} .
\end{aligned}
$$

With the purpose of checking the stability of the $B$-dependent configuration with respect to scalar fluctuations, it is important to keep track of the correct signs in the action. First of all, we therefore replace $\left(\tilde{\tau}^{l}\right)^{2} \rightarrow-4\left(\tilde{\tau}^{l}\right)^{2}$ such that the fluctuations $\tilde{\tau}^{l}=\frac{\tilde{\tau}^{0} \pm \tilde{\tau}^{3}}{\sqrt{2}}$ are now written in terms of the real components of the scalar fluctuation $\tilde{\tau}=\tilde{\tau}^{a} \sigma^{a}$ (where in (4.19) it was implicitly assumed in evaluating the STr that $\tilde{\tau}=\tilde{\tau}^{a} t^{a}=-i \tilde{\tau}^{a} \sigma^{a} / 2$ with imaginary components $\tilde{\tau}^{a}$ ). Slightly redefining $\mathcal{L}_{\text {scalar }}$ to incorporate the sign of the full action,

$$
\left.S_{D B I}\right|_{\tilde{\tau}^{2}}=-\left.T_{8} \int d^{4} x 2 \int_{u_{0}}^{\infty} d u \int \epsilon_{4} e^{-\phi} \mathrm{S} \operatorname{Tr} \sqrt{-\operatorname{det} a}\right|_{\tilde{\tau}^{2}}=T_{8} \int d^{4} x 2 \int_{u_{0}}^{\infty} d u \int \epsilon_{4} \mathcal{L}_{\text {scalar }}
$$

we then end up with

$$
\mathcal{L}_{\text {scalar }}=-\sum_{l=u, d}\left\{I_{l}\left(\bar{x} G_{u u}^{-2}\right)\left(\partial_{u} \tilde{\tau}^{l}\right)^{2}+I_{l}\left(\bar{x} g_{\mu \mu}^{-1} G_{u u}^{-1}\right)\left(D_{\mu} \tilde{\tau}^{l}\right)^{2}+I_{l}\left(\bar{x} g_{\mu \mu}^{-1} G_{u u}^{-1} \frac{1-A}{A}\right) \sum_{\mu=1}^{2}\left(D_{\mu} \tilde{\tau}^{l}\right)^{2}\right\}
$$

with the convention $\left(\partial_{\mu} \tilde{\tau}^{l}\right)^{2}=\partial_{\mu} \tilde{\tau}^{l} \partial_{\nu} \tilde{\tau}^{l} \eta^{\mu \nu}$. 
The Hamiltonian associated with the Lagrangian is given by

$$
\begin{aligned}
& \mathcal{H}=\frac{\delta \mathcal{L}_{\text {scalar }}}{\delta \partial_{0} \tau^{l}} \partial_{0} \tau^{l}-\mathcal{L}_{\text {scalar }} \\
& =\sum_{l=u, d}\left\{I_{l}\left(\bar{x} G_{u u}^{-2}\right)\left(\partial_{u} \tilde{\tau}^{l}\right)^{2}+I_{l}\left(\bar{x} g_{\mu \mu}^{-1} G_{u u}^{-1}\right)\left(\left(\partial_{0} \tilde{\tau}^{l}\right)^{2}+\left(\partial_{3} \tilde{\tau}^{l}\right)^{2}\right)+I_{l}\left(\bar{x} g_{\mu \mu}^{-1} G_{u u}^{-1} A^{-1}\right) \sum_{i=1}^{2}\left(D_{i} \tilde{\tau}^{l}\right)^{2}\right\}
\end{aligned}
$$

where we switched notation again to normal squares $\left(\partial_{\mu} \tau^{l}\right)^{2}=\partial_{\mu} \tau^{l} \partial_{\mu} \tau^{l}$. For the embedding to be stable towards scalar $\tilde{\tau}^{l}$-fluctuations, the associated energy density has to obey

$$
\mathcal{E}=\int_{u_{0, d}}^{\infty} \mathcal{H} \geq 0
$$

which will be the case if each of the $I_{l}$-functions is positive.

Let us discuss the two background functions that appear in the $I_{l}$-functions, $A\left(\bar{F}_{12}\right)$ and $G_{u u}(\partial \bar{\tau})$. Using (3.18), the uu-component of the induced metric on the D8-branes as a function of the embedding $\partial \bar{\tau}$ reads

$$
G_{u u}\left(\partial \bar{\tau}^{0} \pm \partial \bar{\tau}^{3}\right)=G_{u u}\left(\partial \bar{\tau}_{l}\right)=g_{u u}+g_{\tau \tau}\left(\partial_{u} \bar{\tau}_{l}\right)^{2}=\left(\frac{R}{u}\right)^{3 / 2} \frac{1}{f} \frac{1}{1-\frac{u_{0, l}^{8} f_{0, l} A_{0, l}}{u^{8} f A_{l}}}, \quad(l=u, d)
$$

with $u \geq u_{0, l}$ implicitly understood, and, from (3.19),

$$
A\left(F^{0} \pm F^{3}\right)=A_{l}=1+\left(2 \pi \alpha^{\prime}\right)^{2} \bar{F}_{l}^{2}\left(\frac{R}{u}\right)^{3}, \quad(l=u, d)
$$

with the plus (minus) sign corresponding to $l=u(l=d) . A_{l}$ is an increasing function of $B$, equal to 1 for $B=0$, and a decreasing function of $u$, equal to 1 for $u=\infty$ so

$$
A_{l} \geq 1 \quad(\text { for all } B \text { and } u)
$$

The function $1-\frac{u_{0, l}^{8} f_{0, l} A_{0, l}}{u^{8} f A_{l}}$ is a monotonically increasing function of $u$ going from 0 at $u_{0, l}$ to 1 at $u \rightarrow \infty$ for any fixed value of $B$, see figure 4 . Then,

$$
\begin{aligned}
I_{l}\left(\bar{x} G_{u u}^{-2}\right) & =e^{-\phi} g_{11}^{2} g_{S_{4}}^{2} \underbrace{I_{l}\left(G_{u u}^{-3 / 2} A^{1 / 2}\right)}_{G_{u u}^{-3 / 2}\left(\partial \bar{\tau}_{l}\right) A_{l}^{1 / 2}\left(\bar{F}_{l}\right)} \\
& \sim \underbrace{\left(\frac{u_{0}}{R}\right)^{3 / 2} u_{0}^{4} \cdots \infty} \underbrace{f^{3 / 2}}_{\left.\left(1-\frac{u}{R}\right)^{3 / 2} u^{4}\right)^{3 / 2} \cdots 1} \underbrace{\left(1-\frac{u_{0, l}^{8} f_{0, l} A_{0} l}{u^{8} f A_{l}}\right)^{3 / 2}}_{0 \cdots 1 \text { for any fixed value of } B} \underbrace{A_{l}^{1 / 2}}_{\geq 1} \\
\geq & 0, \\
I_{l}\left(\bar{x} g_{11}^{-1} G_{u u}^{-1}\right) & =e^{-\phi} g_{11}^{2} g_{S_{4}}^{2} g_{11}^{-1} \underbrace{I_{l}\left(G_{u u}^{-1 / 2} A^{1 / 2}\right)}_{G_{u u}^{-1 / 2}\left(\partial \bar{\tau}_{l}\right) A_{l}^{1 / 2}\left(\bar{F}_{l}\right)}
\end{aligned}
$$




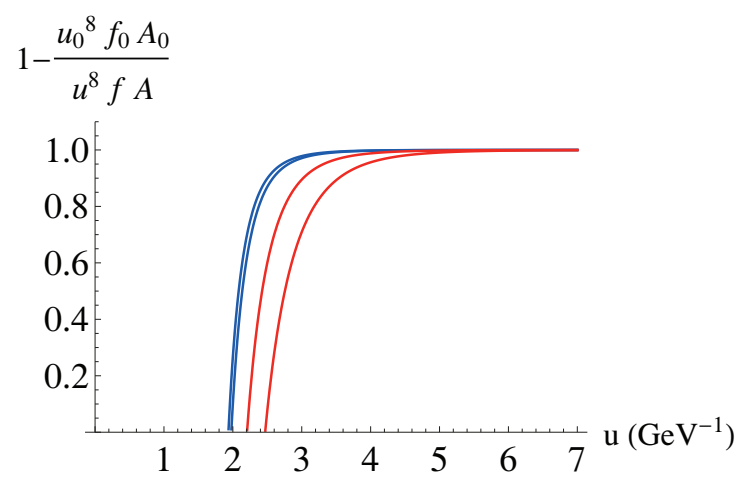

Figure 4. The function $1-\frac{u_{0, l}^{8} f_{0, l} A_{0, l}}{u^{8} f A_{l}}$ as a function of $u$ for $l=u, d$ for $B=0.2 \mathrm{GeV}^{2}$ in blue and $B=1.2 \mathrm{GeV}^{2}$ in red. Up distinguishable from down through $u_{0, u}>u_{0, d}$.

$$
\begin{aligned}
& \sim\left(\frac{u}{R}\right)^{-3 / 4} u^{4}\left(\frac{u}{R}\right)^{-3 / 2}\left(\frac{R}{u}\right)^{-3 / 4} f^{1 / 2}\left(1-\frac{u_{0, l}^{8} f_{0, l} A_{0, l}}{u^{8} f A_{l}}\right)^{1 / 2} A_{l}^{1 / 2} \\
& \sim \underbrace{\left(1-\frac{u_{0, l}^{8} f_{0, l} A_{0, l}}{u^{8} f A_{l}}\right)^{1 / 2}}_{u^{5 / 2} f^{1 / 2}} \underbrace{A_{l}^{1 / 2}}_{0 \cdots 1 \text { for any fixed value of } B} \underbrace{\left(1-u_{K}^{3} u_{0}^{2} \cdots \infty\right.}_{\geq 1} \\
& \geq 0,
\end{aligned}
$$

and for the same reasons

$$
I_{l}\left(\bar{x} g_{11}^{-1} G_{u u}^{-1} A^{-1}\right)=e^{-\phi} g_{11}^{2} g_{S_{4}}^{2} g_{11}^{-1} \underbrace{I_{l}\left(G_{u u}^{-1 / 2} A^{-1 / 2}\right)}_{G_{u u}^{-1 / 2}\left(\partial \bar{\tau}_{l}\right) A_{l}^{-1 / 2}\left(\bar{F}_{l}\right)} \geq 0 .
$$

This concludes the proof of stability of the flavour branes' embedding as depicted in figure 2 with respect to diagonal $\tilde{\tau}$-fluctuations. Note that the off-diagonal $\tilde{\tau}$-components have disappeared through the gauge fixing in section 4.1 - except for an irrelevant mass term for the undynamical $\tilde{\tau}^{1,2}$ in $\mathcal{L}_{\text {Higgs }}$. A similar mechanism in the context of the holographic description of heavy-light mesons can be found in [65].

Let us briefly expand on the physical interpretation of the discussion of stability in the scalar sector. While in the seminal work of [2] (the $x^{\mu}$-dependent parts of) the scalar modes $\tilde{\tau}$ were identified with scalar mesons in the dual field theory, this interpretation was revisited in [66], where it is argued that the $\tilde{\tau}$-fluctuations are to be regarded as artifacts of the SSM. ${ }^{4}$ The reason is that they transform under a $\mathbb{Z}_{2}$-symmetry of the geometric configuration (strictly speaking in the antipodal set-up), which is redundant in the sense that it is not shared with QCD. This is similar to the gauge field components $A_{\Omega_{4}}$ not having a counterpart in the dual QCD-like field theory, as they transform under the $\mathrm{SO}(5)$ isometry of the four-sphere in the background (3.1). Any concern about the interpretation of the off-diagonal $\tilde{\tau}$-components disappearing in the holographic Higgs mechanism coupled

\footnotetext{
${ }^{4}$ We would like to thank S. Sugimoto for private communication about this.
} 
to the gauge fixing, is hence resolved: the 'eaten' fluctuations do not correspond to physical QCD-particles. The above discussion of the stability is not to be interpreted in terms of mesons in the dual field theory, but rather establishes that the geometrical configuration we will employ further is stable against small perturbations.

\subsection{Vector sector in $\left(2 \pi \alpha^{\prime}\right)^{2} F^{2}$-approximation}

Consider the vector part of the DBI-Lagrangian (4.30),

$$
\begin{aligned}
\mathcal{L}= & \mathcal{L}_{\text {Higgs }}+\mathcal{L}_{\text {vector }}=\left.\operatorname{STr} e^{-\phi} \sqrt{-\operatorname{det} a}\right|_{\tilde{A}^{2}} \\
= & \sum_{a=1}^{2}\left\{\gamma(u) \frac{1}{2}\left(\left[\tilde{A}_{u}, \bar{\tau}\right]^{a}\right)^{2}+\alpha(u) \frac{1}{2}\left(\left[\tilde{A}_{\mu}, \bar{\tau}\right]^{a}\right)^{2}+\beta(u) \sum_{\mu=1}^{2} \frac{1}{2}\left(\left[\tilde{A}_{\mu}, \bar{\tau}\right]^{a}\right)^{2}\right\} \\
& +\operatorname{STr} \bar{x}\left\{-\bar{F}_{12} g_{11}^{-2} A^{-1}\left[\tilde{A}_{1}, \tilde{A}_{2}\right]-\left.\frac{1}{4} g_{11}^{-2} \tilde{F}_{\mu \nu}^{2} A^{-2}\right|_{\mu, \nu=1,2}-\left.\frac{1}{2} g_{11}^{-1} G_{u u}^{-1} \tilde{F}_{\mu u}^{2} A^{-1}\right|_{\mu=1,2}\right\} .
\end{aligned}
$$

We have anticipated the vanishing of $\mathcal{L}_{\text {vector-mixing }}$ upon filling in the gauge field expansion in terms of vector mesons, which we will come back to shortly. Let us reinstate the factors $\left(2 \pi \alpha^{\prime}\right)$ that we absorbed into the field strengths for notational convenience, and further approximate $^{5}$ the action to second order in $\left(2 \pi \alpha^{\prime}\right)^{2} \sim 1 / \lambda^{2}$ :

$$
\begin{gathered}
\mathcal{L} \sim u^{1 / 4}\left(2 \pi \alpha^{\prime}\right)^{2} \sum_{a, b=1}^{2}\left\{-\frac{1}{4} f_{1}\left(\tilde{F}_{\mu \nu}^{a}\right)^{2}-\frac{1}{2} g_{11} f_{2}\left(\tilde{F}_{\mu u}^{a}\right)^{2}-\frac{1}{2} \frac{g_{11}}{\left(2 \pi \alpha^{\prime}\right)^{2}}\left(f_{2}-\frac{1}{2} g_{11}^{-2}\left(2 \pi \alpha^{\prime}\right)^{2} f_{3}\right)\left(\tilde{A}_{\mu}^{a}\right)^{2}\left(2 \bar{\tau}^{3}\right)^{2}\right. \\
\quad+\sum_{\mu=1}^{2}\left(-\frac{1}{2} g_{11}^{-1} f_{3}\left(\tilde{A}_{\mu}^{a}\right)^{2}\left(2 \bar{\tau}^{3}\right)^{2}-\frac{1}{2}\left(\sqrt{G_{u u}} \bar{F}_{\mu \nu}\right)^{3} \epsilon_{3 a b} \tilde{A}_{\mu}^{a} \tilde{A}_{\nu}^{b}\right) \\
\left.-\frac{1}{2} \frac{g_{11}^{2}}{\left(2 \pi \alpha^{\prime}\right)^{2}}\left(f_{4}-\frac{1}{2} g_{11}^{-2}\left(2 \pi \alpha^{\prime}\right)^{2} f_{5}\right)\left(\tilde{A}_{u}^{a}\right)^{2}\left(2 \bar{\tau}^{3}\right)^{2}\right\}
\end{gathered}
$$

with proportionality factor $-\frac{1}{2} g_{s}^{-1} R^{\frac{3}{4}+3}$ and

$$
f_{1}=I\left(G_{u u}^{1 / 2}\right), f_{2}=I\left(G_{u u}^{-1 / 2}\right), f_{3}=I\left(G_{u u}^{-1 / 2} \bar{F}_{12}^{2}\right), f_{4}=I\left(G_{u u}^{-3 / 2}\right) \text { and } f_{5}=I\left(G_{u u}^{-3 / 2} \bar{F}_{12}^{2}\right)
$$

similar $I$-functions as encountered in section 4.1.1, again arising from the evaluation of the STr using the prescriptions in appendix A.

Effective 4-dimensional meson fields are introduced via the assumption that the flavour gauge field can be expanded in complete sets $\left\{\psi_{n}(u)\right\}_{n \geq 1}$ and $\left\{\phi_{n}(u)\right\}_{n \geq 0}$ as follows [2]

$$
\begin{aligned}
& A_{\mu}\left(x^{\mu}, u\right)=\sum_{n \geq 1} B_{\mu}^{(n)}\left(x^{\mu}\right) \psi_{n}(u)=\rho_{\mu}\left(x^{\mu}\right) \psi(u)+\cdots \\
& A_{u}\left(x^{\mu}, u\right)=\sum_{n \geq 0} \phi_{\mu}^{(n)}\left(x^{\mu}\right) \phi_{n}(u)=\pi\left(x^{\mu}\right) \phi_{0}(u)+\cdots
\end{aligned}
$$

The rho meson appears as the lowest mode of the infinite vector meson tower $B_{\mu}^{(n)}$, and the pion as the lowest mode of the infinite (pseudo)scalar meson tower $\phi_{\mu}^{(n)}$. We will only retain these lowest-lying mesons in the fluctuation towers, as - with the purpose of

\footnotetext{
${ }^{5}$ We assume here that the expansion in $1 / \lambda$ is justified because $\lambda \approx 15$ is still large for the parameters that we fixed in section 3.2. We will elaborate on the validity of this expansion in the next section.
} 
discussing a possible tachyonic vector instability - it makes sense that the least massive vector meson will likely be the first to condense.

One obtains an effective 4-dimensional action for the mesons by plugging the above fluctuation expansion for the gauge field into the 5-dimensional DBI-action governing the dynamics of the flavour gauge field, and subsequently integrating out the $u$-dependence. Some terms can already be understood to vanish during the integration over the extra radial dimension $u$ by looking at the parity of $\psi(z) \equiv \psi(u(z))$ and $\phi_{0}(z) \equiv \phi_{0}(u(z))$, with $u(z)=u_{0}^{3}+u_{0} z^{2}$ a commonly used coordinate transformation to the coordinate $z=-\infty \cdots \infty$ following the brane from one asymptotic endpoint to the other. Both $\psi(z)$ and $\phi_{0}(z)$ are even functions [2], hence coupling terms between rho mesons and pions of the form $\sim D_{\mu} \tilde{A}_{u}^{a} \partial_{u} \tilde{A}_{\mu}^{a} \sim D_{\mu} \pi^{a} \rho_{\mu}^{a} \phi_{0}(u) \partial_{u} \psi$ originating from $\left(\tilde{F}_{\mu u}^{a}\right)^{2}$ will give rise to vanishing integrals $\int_{-\infty}^{\infty} d z\{$ odd function of $z\}=0$. This means we can discuss the rho meson and the pion terms separately. For the same reason the terms $\sim \tilde{A}_{i} \partial_{u} \tilde{A}_{j}$ (with $i, j=1,2$ )

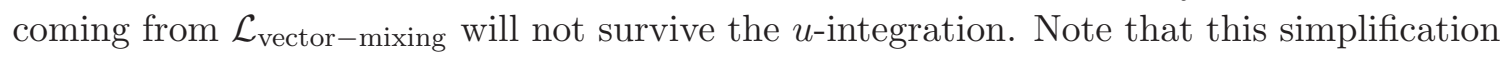
is a consequence of cutting the meson towers down to their lowest states.

\subsubsection{Rho meson mass and rho meson condensation}

Background dependent functions in the action. Before continuing with the strategy outlined above to extract the 4-dimensional effective action for the rho mesons, we take a closer look at the relevant functions $f_{1}, f_{2}$ and $f_{3}$ as defined in (4.42), as well as the definitions for $\bar{\tau}^{3}$ and $\left(G_{u u}^{1 / 2} \bar{F}_{12}\right)^{3}$ in terms of up- and down-components of the background fields.

Using (A.2) and (A.7), we have

$$
\begin{gathered}
f_{1}=I\left(G_{u u}^{1 / 2}\right)=\frac{1}{2\left(\partial \bar{\tau}_{u}-\partial \bar{\tau}_{d}\right)}\left(\sqrt{G_{u u}^{u}} \partial \bar{\tau}_{u}-\sqrt{G_{u u}^{d}} \partial \bar{\tau}_{d}+\frac{g_{u u}}{\sqrt{g_{\tau \tau}}} \ln \left[\frac{\partial \bar{\tau}_{u} g_{\tau \tau}+\sqrt{g_{\tau \tau} G_{u u}^{u}}}{\partial \bar{\tau}_{d} g_{\tau \tau}+\sqrt{g_{\tau \tau} G_{u u}^{d}}}\right]\right) \\
f_{2}=I\left(G_{u u}^{-1 / 2}\right)=\frac{1}{\left(\partial \bar{\tau}_{u}-\partial \bar{\tau}_{d}\right) \sqrt{g_{\tau \tau}}} \ln \left[\frac{\partial \bar{\tau}_{u} g_{\tau \tau}+\sqrt{g_{\tau \tau} G_{u u}^{u}}}{\partial \bar{\tau}_{d} g_{\tau \tau}+\sqrt{g_{\tau \tau} G_{u u}^{d}}}\right] \\
f_{3}=I\left(G_{u u}^{-1 / 2} \bar{F}_{12}^{2}\right)=\frac{1}{2\left(\partial \bar{\tau}_{u}-\partial \bar{\tau}_{d}\right)^{3} g_{\tau \tau}^{3 / 2}}\left\{( \overline { F } _ { d } - \overline { F } _ { u } ) \left[\sqrt{g_{\tau \tau} G_{u u}^{d}}\left(\partial \bar{\tau}_{d} \bar{F}_{d}+3 \partial \bar{\tau}_{d} \bar{F}_{u}-4 \partial \bar{\tau}_{u} \bar{F}_{d}\right)\right.\right. \\
\left.+\sqrt{g_{\tau \tau} G_{u u}^{u}}\left(\partial \bar{\tau}_{u} \bar{F}_{u}+3 \partial \bar{\tau}_{u} \bar{F}_{d}-4 \partial \bar{\tau}_{d} \bar{F}_{u}\right)\right] \\
\left.-\left(2\left(\partial \bar{\tau}_{u} \bar{F}_{d}-\partial \bar{\tau}_{d} \bar{F}_{u}\right)^{2} g_{\tau \tau}-\left(\bar{F}_{d}-\bar{F}_{u}\right)^{2} g_{u u}\right)\left[\ln g_{\tau \tau} g_{u u}+\ln \left(\frac{\partial \bar{\tau}_{u} g_{\tau \tau}+\sqrt{g_{\tau \tau} G_{u u}^{u}}}{\partial \bar{\tau}_{d} g_{\tau \tau}-\sqrt{g_{\tau \tau} G_{u u}^{d}}}\right)\right]\right\}
\end{gathered}
$$

with $\partial \bar{\tau}$ short for $\partial_{u} \bar{\tau}=(3.18), G_{u u}^{l}=G_{u u}\left(\partial_{u} \bar{\tau}_{l}\right)$ and $\bar{F}_{u}=\frac{2 B}{3}, \bar{F}_{d}=-\frac{B}{3}$, as defined in (3.15). Because of the theta-functions $\theta\left(u-u_{0, l}\right)$ contained in $\partial_{u} \bar{\tau}_{l}$, the contribution of $\partial_{u} \bar{\tau}_{u}$ only kicks in at $u>u_{0, u}$. Therefore these functions will all be discontinuous at $u=u_{0, u}$, as can be seen in the illustrative plot in figure 5 for $B=0.8 \mathrm{GeV}^{2}$. The dependence on $B$ is implicit through the embedding, except for $f_{3}$ which also depends explicitly on $B$. Further, $\bar{\tau}^{3}$ gives a measure for the distance between up- and down-brane, defined as

$$
\bar{\tau}^{3}(u)=\int_{\infty}^{u} \partial_{u} \bar{\tau}^{3} d u=\int_{\infty}^{u} \frac{\partial_{u} \bar{\tau}_{u}-\partial_{u} \bar{\tau}_{d}}{2} d u=\int_{\infty}^{u_{0, u}} \frac{\partial_{u} \bar{\tau}_{u}-\partial_{u} \bar{\tau}_{d}}{2} d u+\int_{u_{0, u}}^{u} \frac{-\partial_{u} \bar{\tau}_{d}}{2} d u
$$

such that $\bar{\tau}^{3}$ fulfills the boundary condition that the flavour branes coincide at $u \rightarrow \infty$ : $\bar{\tau} \sim \mathbb{1} \Rightarrow \bar{\tau}^{3}(\infty)=0$. In figure 6 the resulting discontinuous $\bar{\tau}^{3}$ is plotted for $B=0.8 \mathrm{GeV}^{2}$, 

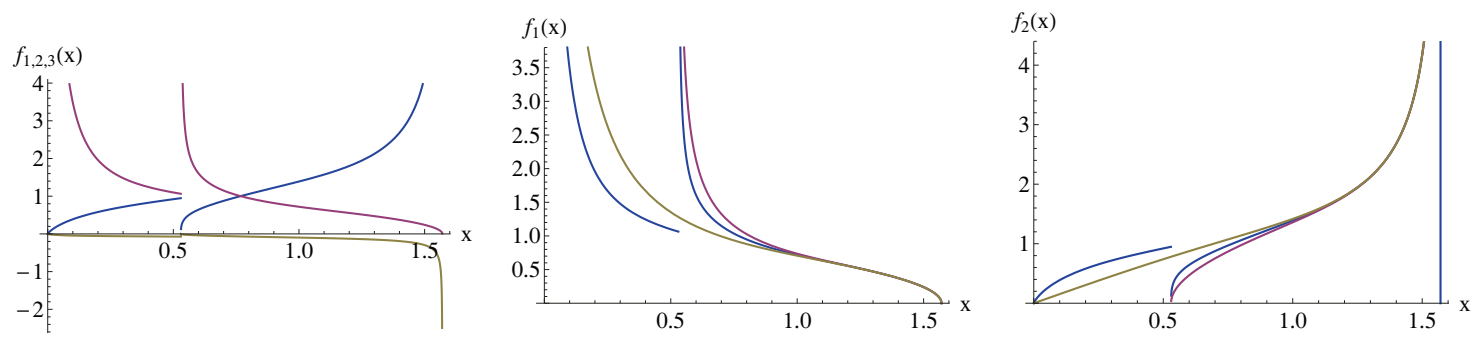

Figure 5. (a) $f_{1}$ (red), $f_{2}$ (blue) and $f_{3}$ (yellow) plotted as functions of $x$, related to $u$ through $u=u_{0, d} \cos ^{-3 / 2} x$ mapping the infinite $u$-range to $x=0 \cdots \pi / 2$. (b) $f_{1}=I\left(G_{u u}^{1 / 2}\right)$ (blue) compared to $\left(G_{u u}^{d}\right)^{1 / 2}$ (yellow) and $\left(G_{u u}^{u}\right)^{1 / 2}$ (red), i.e. the functions which would replace $f_{1}$ if there were a $\operatorname{Tr}$ instead of a STr in the action, reducing the non-Abelian to a sum of two Abelian actions. As required, $f_{1} \rightarrow G_{u u}^{1 / 2}$ in the limit of coinciding branes at $u \rightarrow \infty$. (c) $f_{2}=I\left(G_{u u}^{-1 / 2}\right)$ (blue) compared to $\left(G_{u u}^{d}\right)^{-1 / 2}$ (yellow) and $\left(G_{u u}^{u}\right)^{-1 / 2}$ (red). All plots for $B=0.8 \mathrm{GeV}^{2}$.

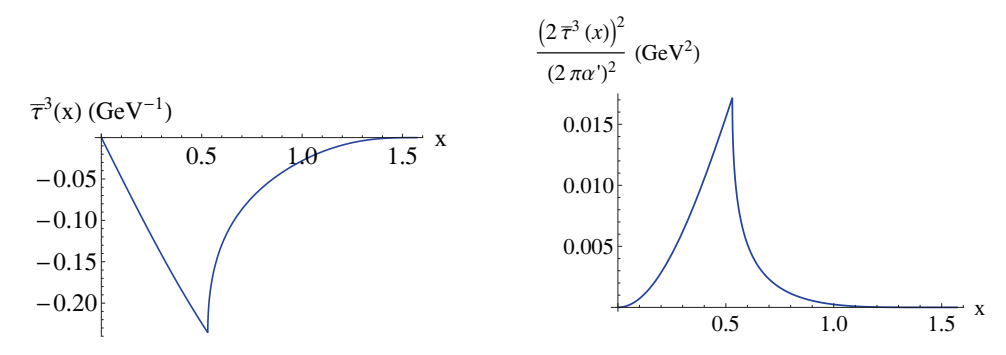

Figure 6. The measure $\bar{\tau}^{3}(x)$ for the splitting of the branes and the resulting estimated contribution to the mass term for the flavour gauge field and indirectly the rho meson. The range $x=0 \cdots \pi / 2$ maps to $u=u_{0, d} \cdots \infty$ and we chose $B=0.8 \mathrm{GeV}^{2}$.

along with $\left(2 \bar{\tau}^{3}\right)^{2} /\left(2 \pi \alpha^{\prime}\right)^{2}$ which contributes to the ' $u$-dependent mass' of the 5-dimensional gauge field. The contribution is small - although it is $\left(2 \pi \alpha^{\prime}\right)^{-2}$-enhanced - since the splitting itself is a small effect. The last relevant background function in the action (4.41) for the discussion of the rho mesons is

$$
\left(G_{u u}^{1 / 2} \bar{F}_{12}\right)^{3}=\sqrt{G_{u u}^{u}} \bar{F}_{u}-\sqrt{G_{u u}^{d}} \bar{F}_{d} .
$$

Eigenvalue problem. Upon substitution of the gauge field expansions (4.43) and (4.44) into (4.41), the 5-dimensional DBI-Lagrangian to second order in the rho meson fluctuations (and second order in $\left(2 \pi \alpha^{\prime}\right)$ ) reads

$$
\begin{aligned}
\mathcal{L} \sim u^{1 / 4}\left(2 \pi \alpha^{\prime}\right)^{2} \sum_{a, b=1}^{2} & \left\{-\frac{1}{4} f_{1}\left(\mathcal{F}_{\mu \nu}^{a}\right)^{2} \psi^{2}-\frac{1}{2} g_{11} f_{2}\left(\rho_{\mu}^{a}\right)^{2}\left(\partial_{u} \psi\right)^{2}-\frac{1}{2} \frac{g_{11}}{\left(2 \pi \alpha^{\prime}\right)^{2}} \tilde{f}_{2}\left(\rho_{\mu}^{a}\right)^{2} \psi^{2}\left(2 \bar{\tau}^{3}\right)^{2}\right. \\
& \left.+\sum_{\mu=1}^{2}\left(-\frac{1}{2} g_{11}^{-1} f_{3}\left(\rho_{\mu}^{a}\right)^{2} \psi^{2}\left(2 \bar{\tau}^{3}\right)^{2}-\frac{1}{2}\left(\sqrt{G_{u u}} \bar{F}_{\mu \nu}\right)^{3} \epsilon_{3 a b} \rho_{\mu}^{a} \rho_{\nu}^{b} \psi^{2}\right)\right\}+ \text { pions }
\end{aligned}
$$

with $\mathcal{F}_{\mu \nu}^{a}=D_{\mu} \rho_{\nu}^{a}-D_{\nu} \rho_{\mu}^{a}$ and $\tilde{f}_{2}=f_{2}-\frac{1}{2} g_{11}^{-2}\left(2 \pi \alpha^{\prime}\right)^{2} f_{3}$. 
Demanding that the first line of this Lagrangian reduces to the standard 4-dimensional form

$$
\sum_{a=1,2}\left(-\frac{1}{4}\left(\mathcal{F}_{\mu \nu}^{a}\right)^{2}-\frac{1}{2} m_{\rho}^{2}\left(\rho_{\mu}^{a}\right)^{2}\right)
$$

after integrating out the $u$-dependences, leads to a normalization condition

$$
\int_{u_{0, d}}^{\infty} d u u^{1 / 4} f_{1} \psi^{2}=1
$$

and a mass term condition

$$
\int_{u_{0, d}}^{\infty} d u\left\{u^{1 / 4} g_{11} f_{2}\left(\partial_{u} \psi\right)^{2}+u^{1 / 4} \frac{g_{11}}{\left(2 \pi \alpha^{\prime}\right)^{2}} \tilde{f}_{2}\left(2 \bar{\tau}^{3}\right)^{2} \psi^{2}\right\}=m_{\rho}^{2}
$$

on the $\psi(u)$ functions, ${ }^{6}$ which combine through partial integration to an eigenvalue equation for $\psi(u)$ :

$$
u^{-1 / 4} f_{1}^{-1} \partial_{u}\left(u^{1 / 4} g_{11} f_{2} \partial_{u} \psi\right)-\frac{g_{11}}{\left(2 \pi \alpha^{\prime}\right)^{2}} f_{1}^{-1} \tilde{f}_{2}\left(2 \bar{\tau}^{3}\right)^{2} \psi=-\Lambda \psi,
$$

with the eigenvalue $\Lambda=m_{\rho}^{2}$ the sought for rho meson mass squared. We can separate the Higgs contribution to $m_{\rho}^{2}$ by defining

$$
\tilde{m}_{\rho}^{2}=\int_{u_{0, d}}^{\infty} d u u^{1 / 4} g_{11} f_{2}\left(\partial_{u} \psi\right)^{2} \quad \text { and } \quad m_{\rho, \text { Higgs }}^{2}=\int_{u_{0, d}}^{\infty} d u u^{1 / 4} \frac{g_{11}}{\left(2 \pi \alpha^{\prime}\right)^{2}} \tilde{f}_{2}\left(2 \bar{\tau}^{3}\right)^{2} \psi^{2}
$$

such that

$$
m_{\rho}^{2}=\tilde{m}_{\rho}^{2}+m_{\rho, \text { Higgs }}^{2} .
$$

Let us also mention that from (4.52) one can see that $m_{\rho}^{2}>0$.

To solve the eigenvalue equation (4.53) numerically on a compact interval, we change to the coordinate $x=0 \cdots x_{u p} \cdots \frac{\pi}{2}$ related to $u=u_{0, d} \cdots u_{0, u} \cdots \infty$ by

$$
u^{3}=u_{0, d}^{3} \cos ^{-2} x
$$

Rewritten as a function of $x$, the eigenvalue equation is invariant under $x \rightarrow-x$, so we can split up the eigenfunction set in even/odd $\psi_{n}(x)$ 's, which correspond to odd/even parity mesons:

$$
\psi_{n}(0)=0 \quad \text { or } \quad \partial_{x} \psi_{n}(0)=0 .
$$

Asymptotically, the eigenvalue equation (4.53) reduces to $\partial_{u}\left(u^{5 / 2} \partial_{u} \psi\right)=0$, with the asymptotic solution $\psi(\infty)=c \frac{u^{-3 / 2}}{-3 / 2}+d$ only normalizable through $(4.51)$ if $d=0$, i.e. if

$$
\psi(u \rightarrow \infty)=0 \quad \text { or } \quad \psi(x \rightarrow \pm \pi / 2)=0 .
$$

\footnotetext{
${ }^{6}$ We absorbed the total prefactor $\sqrt{V_{4} T_{8} g_{s}^{-1} R^{\frac{3}{4}+3}\left(2 \pi \alpha^{\prime}\right)^{2}}$ into $\psi$ such that $\psi$ has a total mass dimension of $5 / 8$ instead of 2 (without the prefactor).
} 
The eigenvalue problem (4.53) for the (odd parity) rho meson with the appropriate boundary condition (4.58) in the $x$-coordinate is thus of the form

$$
\cdots \partial_{x}^{2} \psi+\cdots \partial_{x} \psi+\cdots \psi=-\Lambda \psi \quad \text { with } \quad \psi( \pm \pi / 2)=0, \quad \partial_{x} \psi(0)=0 .
$$

To solve it we employ a shooting method, which consists of temporarily replacing (4.59) with the well-defined initial value problem

$$
\cdots \partial_{x}^{2} \psi+\cdots \partial_{x} \psi+\cdots \psi=-\Lambda \psi \quad \text { with } \quad \psi(0)=1, \quad \partial_{x} \psi(0)=0
$$

where $\Lambda$ is treated as a 'shooting' parameter. We used the scaling freedom $\psi(x) \rightarrow h \psi(x)$ to impose that $\psi(0)=1$ (the value of $h$ will be fixed by the normalization condition in the end). For each value of $\Lambda,(4.60)$ can be solved numerically for $\psi_{\Lambda}(x)$. Next, solving the equation $\psi_{\Lambda}(\pi / 2)=0$ finally determines the eigenvalue $\Lambda=m_{\rho}^{2}$.

For completeness we add a few comments about the numerical method we used to solve the eigenvalue problem at hand (4.59), which in detail reads

$$
\frac{9}{4} R^{-3 / 2} u_{0, d}^{-1 / 2} \frac{\cos ^{11 / 6} x}{\sin x} f_{1}^{-1} \partial_{x}\left(f_{2} \frac{\cos ^{1 / 2} x}{\sin x} \partial_{x} \psi\right)-R^{-3 / 2} \frac{u_{0, d}^{3 / 2}}{\left(2 \pi \alpha^{\prime}\right)^{2}}\left(\cos ^{-1} x\right) \tilde{f}_{2} f_{1}^{-1}\left(2 \bar{\tau}^{3}\right)^{2} \psi=-m_{\rho}^{2} \psi,
$$

with $\psi( \pm \pi / 2)=0$ and $\partial_{x} \psi(0)=0$. Near the origin $x \rightarrow 0$ the equation takes the form

$$
m_{\rho}^{2} \psi+\partial_{x}^{2} \psi-\ln x \partial_{x}^{2} \psi-\frac{1}{x} \partial_{x} \psi=0
$$

so we have to provide Mathematica with an ansatz for $\psi(x)$ at small $x$ to prevent the equation from blowing up there. Demanding that $\partial_{x} \psi \sim x$ to avoid the last term in (4.62) from diverging, would still give $\ln x \partial_{x}^{2} \psi \rightarrow-\infty$. Instead we demand that $\partial_{x}^{2} \psi \sim \frac{1}{\ln x}$ or $\psi(x \rightarrow 0)=1+x^{2} \sum_{i=1}^{n} \frac{a_{i}}{\ln ^{i} x}$ (in practice we have set $n=13$ ). With this ansatz for $\psi \Rightarrow \partial_{x} \psi \sim \operatorname{LogIntegral}(x)+c$, the term $\frac{1}{x} \partial_{x} \psi$ will only be finite if the integration constant $c=\partial_{x} \psi(0)=0 .{ }^{7}$ Near $x=x_{u p}$, or $y \rightarrow 0$ in the useful coordinate $y$ defined through $u^{3}=u_{0, u}^{3} \cos ^{-2} y$, the differential equation's form

$$
m_{\rho}^{2} \psi+\partial_{y}^{2} \psi-\ln y \partial_{y}^{2} \psi-\frac{1}{y} \partial_{y} \psi=0
$$

again needs to be fed with an ansatz for $\psi$ that keeps the equation finite, i.e. $\psi(y \rightarrow 0)=\psi\left(x=x_{u p}\right)+y^{2} \sum_{i=1}^{n} \frac{a_{i}}{\ln ^{i} y}$ with $\partial_{y} \psi(0)=0$. This means we can demand continuity of $\psi$ at $x=x_{u p}$ but not of its derivative. ${ }^{8}$ An example result of $\psi(x)$ and its derivative is shown in figure 7 .

\footnotetext{
${ }^{7}$ This is consistent with vector mesons, but not with the initial condition on axial mesons (which we have not considered). We have not looked into it further to see if there is a way around this, in order to still be able to describe axial mesons in the presence of a magnetic field in this setting.

${ }^{8}$ It is known that the Schrödinger wave function can display kinks (thus jumps in its derivative), depending on the potential (singularities), see e.g. [67]. This corresponds to the singular behaviour of some of the coefficient functions for $y \rightarrow 0$ in (4.63).
} 

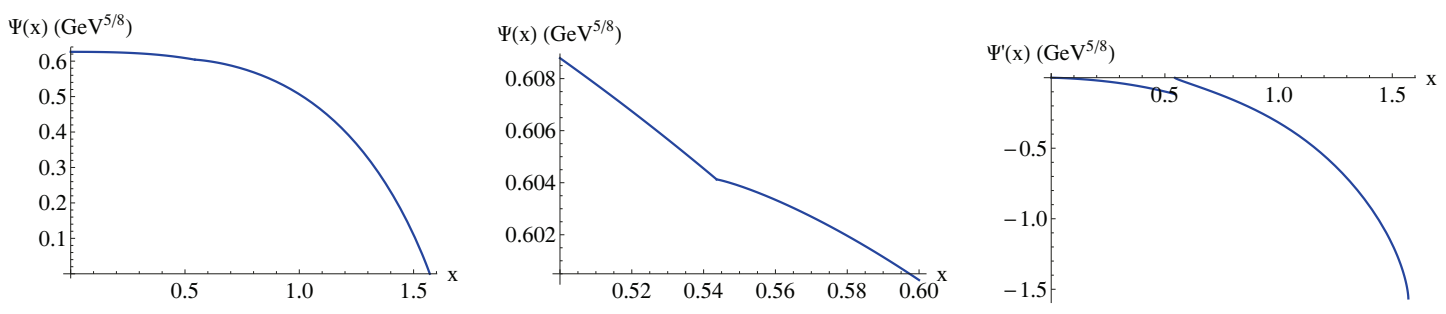

Figure 7. Plots of the rho meson eigenfunction $\psi(x)$ and its derivative $\partial_{x} \psi(x)$, discontinuous at $x=x_{u p} \approx 0.54$, for $B=0.9 \mathrm{GeV}^{2}$, obtained numerically with a shooting method.

Effective 4-dimensional EOM and result for total eigenvalue. The effective 4dimensional action becomes

$$
S_{4 D}=\int d^{4} x \sum_{a, b=1}^{2}\left\{-\frac{1}{4}\left(\mathcal{F}_{\mu \nu}^{a}\right)^{2}-\frac{1}{2} m_{\rho}^{2}(B)\left(\rho_{\mu}^{a}\right)^{2}+\sum_{\mu=1}^{2}\left(-\frac{1}{2} m_{+}^{2}(B)\left(\rho_{\mu}^{a}\right)^{2}-\frac{1}{2} \epsilon_{3 a b} \rho_{\mu}^{a} \rho_{\nu}^{b} k(B) \bar{F}_{\mu \nu}^{3}\right)\right\}
$$

with the normalized $\psi$, as determined in the previous paragraph, satisfying the normalization and mass conditions (4.51) and (4.52), and the newly defined $m_{+}$and $k$ to be calculated from

$$
\int_{u_{0, d}}^{\infty} d u u^{1 / 4} g_{11}^{-1} f_{3}\left(2 \bar{\tau}^{3}\right)^{2} \psi^{2}=m_{+}^{2}
$$

and

$$
\int_{u_{0, d}}^{\infty} d u u^{1 / 4}\left(\sqrt{G_{u u}} \bar{F}_{12}\right)^{3} \psi^{2}=k \bar{F}_{12}^{3}
$$

with $\bar{F}_{12}^{3}=B$. Here $m_{+}$is an extra contribution to the mass of the transverse (w.r.t. the magnetic field $\vec{B}=B \vec{e}_{3}$ ) components of the charged rho meson, $\rho_{\mu=1,2}^{a=1,2}$, as a consequence of $B$ breaking Lorentz invariance. The parameter $k$ describes a non-minimal coupling of the charged rho meson to the magnetic field, related to the magnetic moment $\mu$ via $\mu=(1+k) e /(2 m)$ so to the gyromagnetic ratio $g$ via $g=1+k$.

The standard 4-dimensional action used to describe the coupling of charged rho mesons to an external magnetic field is the Proca action [68] (which is equivalent to the DSGSaction [32] for self-consistent rho meson quantum electrodynamics to second order in the fields). The Proca action is equal to (4.64) with $m_{+}=0$ and $m_{\rho}$ and $k(=1)$ independent of $B$ : there is only explicit dependence of the action on $B$, which is to be traced back to the treatment of the rho mesons as point-like structureless particles. Instead, in our approach, the effect of $B$ on the constituent quarks is taken into account via the effect of $B$ on the embedding of the flavour probe branes, ${ }^{9}$ leading to an implicit dependence on $B$ of both the mass $m_{\rho}^{2}(B)$ and the magnetic coupling $k(B)$. The effect of $B$ on the embedding is two-fold (see section 3.4 and in particular figure 2): the branes move upwards in the holographic direction, corresponding to chiral magnetic catalysis, and the up- and down-brane get separated, corresponding to a stronger chiral magnetic catalysis for the up-quark than for the down-quark. Both effects translate into a mass generating effect for the rho meson,

\footnotetext{
${ }^{9}$ In the antipodal Sakai-Sugimoto model where the embedding is $B$-independent, one recovers exactly the Proca action [1].
} 

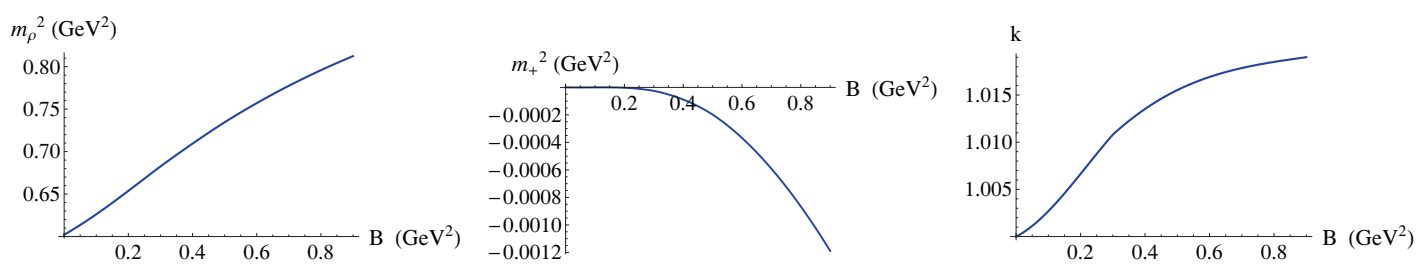

Figure 8. Numerical results for $m_{\rho}^{2}(B), m_{+}^{2}(B)$ and $k(B)$ in the $\left(2 \pi \alpha^{\prime}\right)^{2} F^{2}$-approximation of the DBI-action.

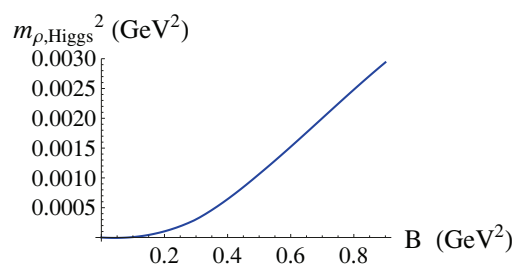

Figure 9. The Higgs contribution $m_{\rho, H i g g s}^{2}(B)$ to the rho meson mass squared $m_{\rho}^{2}(B)$, as defined in (4.54), in the $\left(2 \pi \alpha^{\prime}\right)^{2} F^{2}$-approximation of the DBI-action.

$m_{\rho}^{2}(B) \nearrow$, as can be seen in figure 8 . The chiral magnetic catalysis causes the rho meson to get heavier as its constituents do. The split between the branes adds to the mass of the rho meson via a holographic Higgs mechanism: as the branes separate, the flavour gauge field strings between up and down branes (i.e. representing charged quark-antiquark combinations $u \bar{d}, \bar{u} d$ ) get stretched. Because of their string tension this results in an extra Higgs mass term in the action for $\tilde{A}_{\mu}^{a=1,2}$ - and thus for $\rho_{\mu}^{a=1,2}$ - of the form $\left(A_{\mu}^{a}\right)^{2}\left(\bar{\tau}^{3}\right)^{2}$, with $\bar{\tau}^{3} \sim \bar{\tau}_{u}-\bar{\tau}_{d}$, originating from $\left(D_{\mu} \tau\right)^{2} \rightsquigarrow\left(\left[\tilde{A}_{\mu}, \bar{\tau}\right]\right)^{2}$ in the start action. Where in the absence of splitted branes, $\bar{\tau}^{3}=0$, the 4 -dimensional mass $m_{\rho}$ as defined in going from (4.49) to (4.50) is purely effective, i.e. only present after integrating out the fifth dimension $u$, the Higgs contributions to the mass stem from the stringy mass of the 5-dimensional gauge field itself. A direct interpretation of the stringy mass contribution in effective QCD-terms we cannot offer. Since the splitting of the branes is small though, the induced mass contribution is almost negligible, see figure 9. Further, as can be seen in figure $8, m_{+}(B) \searrow$ as $f_{3}$ in (4.65) is negative, so the mass of the transversal components of the charged rho mesons will already be slightly smaller than that of the longitudinal ones, and $k(B) \nearrow$ is approximately equal to one, but not exactly, corresponding to a gyromagnetic ratio $g \approx 2$.

The 4-dimensional EOMs for the charged rho mesons $\rho_{\mu}^{a=1,2}$ are given by

$$
\begin{array}{r}
D_{\mu} \mathcal{F}_{\mu \nu}^{a}-\epsilon_{a 3 b} k(B) \bar{F}_{\mu \nu}^{3} \rho_{\mu}^{b}-M^{2}(B) \rho_{\nu}^{a}=0 \\
M^{2}(B)=m_{\rho}^{2}(B)+\left(\delta_{\nu 1}+\delta_{\nu 2}\right) m_{+}^{2}(B)
\end{array}
$$

with $D_{\mu}=\partial_{\mu}+\left[\bar{A}_{\mu}, \cdot\right]$ and $\mathcal{F}_{\mu \nu}^{a}=D_{\mu} \rho_{\nu}^{a}-D_{\nu} \rho_{\mu}^{a}$. They combine into the EOM

$$
\mathrm{D}_{\mu}\left(\mathrm{D}_{\mu} \rho_{\nu}-\mathrm{D}_{\nu} \rho_{\mu}\right)-i k(B) \bar{F}_{\mu \nu}^{3} \rho_{\mu}-M^{2}(B) \rho_{\nu}=0
$$

with $\mathrm{D}_{\mu}=\partial_{\mu}+i \bar{A}_{\mu}^{3}$ for the charged combination $\rho_{\mu}=\left(\rho_{\mu}^{1}+i \rho_{\mu}^{2}\right) / \sqrt{2}$, and the complex conjugate of this equation for the other charged combination $\rho_{\mu}^{*}=\left(\rho_{\mu}^{1}-i \rho_{\mu}^{2}\right) / \sqrt{2}$. 
Solving (4.69) with $\rho_{\nu} \rightarrow e^{i(\vec{p} \cdot \vec{x}-E t)} \rho_{\nu}$ for the eigenvalues of the energy, one finds 'modified Landau levels' that we will discuss in more detail in the next section, where they will show up as a special case of the most general form of modified Landau levels that we encounter solving the 4-dimensional EOMs that come from the use of the full DBI-action. Only in the case that $k=1, m_{+}=0$ and $m_{\rho}(B)=m_{\rho}(0)$ one retrieves the standard Landau levels for a free relativistic spin- $s$ particle moving in the background of a constant magnetic field $\vec{B}=B \vec{e}_{3}$ (assuming $B>0$ ):

$$
E^{2}=m_{\rho}^{2}+p_{3}^{2}+\left(2 n-2 s_{3}+1\right) B
$$

with $n$ the Landau level number and $s_{3}$ the eigenvalue of the spin operator

$$
S_{3}=\frac{1}{2}\left(\begin{array}{cc}
0 & \sigma_{2}-i \sigma_{1} \\
\sigma_{2}+i \sigma_{1} & 0
\end{array}\right)
$$

giving the projection of the spin of the particle onto the direction of the magnetic field.

While the modifications due to $k \neq 1, m_{+}(B) \neq 0$ and $m_{\rho}(B)$ are a bit subtle for higher levels, the energy of $s_{3}=1, p_{3}=0$ particles in the lowest Landau level $n=0$ is given by a straightforward generalization

$$
E^{2}=M^{2}(B)-B k(B)
$$

of $E^{2}=m_{\rho}^{2}-B$. We conclude that the combinations of charged rho mesons that have their spin aligned with the magnetic field, $s_{3}=1$, i.e.

$$
\rho=\rho_{1}+i \rho_{2} \quad \text { and } \quad \rho^{*}=\rho_{1}^{*}-i \rho_{2}^{*},
$$

will have an effective mass squared

$$
m_{\rho, e f f}^{2}=M^{2}(B)-B k(B)
$$

going through zero at a critical magnetic field

$$
B_{c} \approx 0.78 \mathrm{GeV}^{2} \text {, }
$$

which marks the onset of rho meson condensation. Our result for $m_{\rho, \text { eff }}^{2}$ is shown in figure 10 .

The total action includes, next to the DBI-part, a Chern-Simons term. In general, contributions from the Chern-Simons action are suppressed in the large $\lambda$ expansion, but in the presence of large background fields Chern-Simons effects can become important, similar to the higher order terms in the $\left(2 \pi \alpha^{\prime} \sim \frac{1}{\lambda}\right)$-expansion of the DBI-action (see comments in the upcoming section 4.4.1). The intrinsic-parity-odd nature of the ChernSimons action ensures that it will not contribute $\rho^{2}$-terms to the effective 4 -dimensional action to second order in the fluctuations, but it will describe $\rho \pi B$ coupling terms between rho mesons and pions. However, as discussed in more detail in [1], the antisymmetrization over spacetime indices in the Chern-Simons action

$$
S_{C S} \sim \int \operatorname{Tr}\left(\epsilon^{m n p q r} A_{m} F_{n p} F_{q r}+\mathcal{O}\left(\tilde{A}^{3}\right)\right)
$$




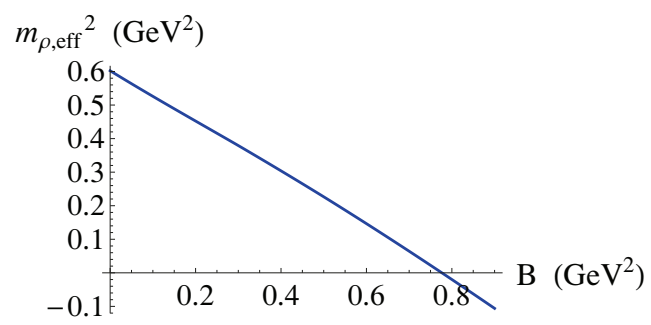

Figure 10. The effective rho meson mass squared $m_{\rho, \text { eff }}^{2}(B)$ in the $\left(2 \pi \alpha^{\prime}\right)^{2} F^{2}$-approximation of the DBI-action.

will make sure that the magnetic field $B=\bar{F}_{12}^{3}$ only induces couplings between longitudinal fluctuations $(\mu=0,3)$, hence not affecting the dynamics of transversal rho mesons (4.73) and their condensation.

\subsubsection{Pion mass}

We briefly discuss the pion part of the DBI-Lagrangian (4.41), which upon substitution of the gauge field expansion (4.44) and further approximation to second order in the pion fields reads

$$
\mathcal{L} \sim u^{1 / 4}\left(2 \pi \alpha^{\prime}\right)^{2} \sum_{a, b=1}^{2}\left\{-\frac{1}{2} g_{11} f_{2}\left(D_{\mu} \pi^{a}\right)^{2} \phi_{0}^{2}-\frac{1}{2} \frac{g_{11}^{2}}{\left(2 \pi \alpha^{\prime}\right)^{2}} \tilde{f}_{4}\left(2 \bar{\tau}^{3}\right)^{2}\left(\pi^{a}\right)^{2} \phi_{0}^{2}\right\}
$$

with $\tilde{f}_{4}=f_{4}-\frac{1}{2} g_{11}^{-2}\left(2 \pi \alpha^{\prime}\right)^{2} f_{5}$. Ignoring in this section the $1 / \lambda$-suppressed $\rho \pi B$ contributions from the Chern-Simons action, the effective 4-dimensional action for the pions becomes

$$
S_{4 D}=\int d^{4} x \sum_{a, b=1}^{2}\left\{-\frac{1}{2}\left(D_{\mu} \pi^{a}\right)^{2}-\frac{1}{2} m_{\pi}^{2}(B)\left(\pi^{a}\right)^{2}\right\}
$$

with $\phi_{0}$ satisfying the normalization condition

$$
\int_{u_{0, d}}^{\infty} d u u^{1 / 4} g_{11} f_{2} \phi_{0}^{2}=1
$$

and the pions no longer massless:

$$
\int_{u_{0, d}}^{\infty} d u u^{1 / 4} \frac{g_{11}^{2}}{\left(2 \pi \alpha^{\prime}\right)^{2}} \tilde{f}_{4}\left(2 \bar{\tau}^{3}\right)^{2} \phi_{0}^{2}=m_{\pi}^{2}
$$

We can understand the emergence of this mass again as a consequence of the holographic Higgs mechanism. The magnetic field breaks chiral symmetry explicitly (albeit only slightly) by pulling the up- and down-brane apart. The previously massless pions, serving as Goldstone bosons associated with the spontaneous breaking of chiral symmetry, hence get a small mass, related to the distance $\bar{\tau}^{3} \sim \bar{\tau}_{u}-\bar{\tau}_{d}$ between the branes. Solving the effective 4-dimensional EOM for the charged pions with $\pi \rightarrow e^{i(\vec{p} \cdot \vec{x}-E t)} \pi$ for the eigenvalues of the energy, one finds 'almost Landau levels' for a spinless particle

$$
E^{2}=m_{\pi}^{2}(B)+p_{3}^{2}+(2 n+1) B
$$



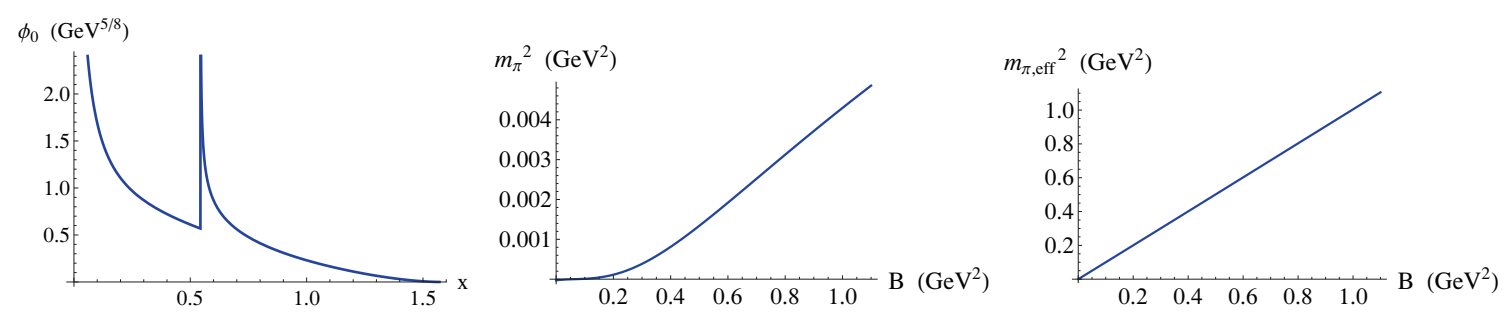

Figure 11. Pion eigenfunction $\phi_{0}(x)$ (with $u=u_{0, d} \cos ^{-3 / 2} x$ ) for $B=1.1 \mathrm{GeV}^{2}$. Numerical result for $m_{\pi}^{2}(B)$ and the effective pion mass squared $m_{\pi, \text { eff }}^{2}(B)$ in the $\left(2 \pi \alpha^{\prime}\right)^{2} F^{2}$-approximation of the DBI-action.

or an effective mass squared in the lowest Landau level

$$
m_{\pi, e f f}^{2}=m_{\pi}^{2}(B)+B
$$

The pion thus gets a mass in the presence of a magnetic field, although we are working in a model in the chiral limit (zero bare quark masses) and with no chiral condensate (at least not in the setting we used, without incorporating a tachyon field as was done in [43]). This violates the GMOR-relation relating the bare quark masses times chiral condensate to the mass of the pion. It was however already discussed in e.g. [47, 69] that the GMOR-relation is no longer valid for charged pions in the presence of a magnetic field.

To calculate the mass $m_{\pi}$ in (4.80), we determine the form of the eigenfunction $\phi_{0}(u)$ analogously as in [2]. $\phi_{0}$ has to be orthogonal to all other $\phi_{n \geq 1}$ (the higher eigenfunctions that we left out in the expansion (4.44)). The eigenfunctions $\phi_{n \geq 1}$ obey the same normalization condition (4.79) as $\phi_{0}$, which upon comparison with the mass condition (4.54) for $\psi_{n \geq 1}$,

$$
\int_{u_{0, d}}^{\infty} d u u^{1 / 4} g_{11} f_{2} \phi_{n \geq 1}^{2}=1 \text { and } \int_{u_{0, d}}^{\infty} d u u^{1 / 4} g_{11} f_{2}\left(\partial_{u} \psi_{n \geq 1}\right)^{2}=\tilde{m}_{\rho}^{2},
$$

leads to

$$
\phi_{n \geq 1}=\frac{\partial_{u} \psi_{n \geq 1}}{\sqrt{\tilde{m}_{\rho}^{2}}} .
$$

Then, orthogonality of $\phi_{0}$ and $\phi_{n \geq 1} \sim \partial_{u} \psi_{n \geq 1}$ is ensured by proposing

$$
\phi_{0} \sim u^{-1 / 4} g_{11}^{-1} f_{2}^{-1}
$$

(with normalization constant determined by the normalization condition (4.79)):

$$
\int_{u_{0, d}}^{\infty} d u \phi_{0}\left(u^{1 / 4} g_{11} f_{2} \phi_{n \geq 1}\right) \sim \int d u \partial_{u} \psi_{n \geq 1}=0
$$

by virtue of the vanishing of $\psi_{n \geq 1}$ at the boundary $u \rightarrow \infty$. With $\phi_{0}$ given in (4.85) we can determine the Higgs contribution to the mass $m_{\pi}$. In figure 11 we plot the eigenfunction $\phi_{0}(u)$ (which is discontinuous due to the discontinuous nature of $f_{2}$ ), the mass $m_{\pi}$ and the total effective 4-dimensional mass $m_{\pi, \text { eff }}$. 
We end this section with a comment on the validity of the use of the non-Abelian DBI-action for non-coincident branes. ${ }^{10}$

In the context of heavy-light mesons, which we encounter here as magnetically induced through the splitting of the flavour branes, one often studies the separated branes system by the use of two (Abelian) DBI-actions plus a Nambu-Goto action for the classical, i.e. macroscopic, heavy-light meson string (e.g. [70]). In [65] however, one uses the non-Abelian DBI action for the description of heavy-light mesons, as we also did in this paper. They do remark that as soon as the distance between the separated branes is larger than the fundamental string length $\ell_{s}$, the non-Abelian DBI-description is actually expected to break down. So let us show here that in our case the separation between up- and down-brane and hence the length of the charged rho meson strings is not larger than $\ell_{s}$.

The total length of a string stretching in the $u$ - and $\tau$-direction is given by

$$
L_{s}=\int d s=\int \sqrt{g_{\tau \tau} d \tau^{2}+g_{u u} d u^{2}} .
$$

Consider for example a string at $\tau=0$ stretching from $u_{0, d}(B)$ to $u_{0, u}(B)$. It has a length

$$
\begin{aligned}
L_{s}= & \int d s=\int_{u_{0, d}(B)}^{u_{0, u}(B)} \sqrt{g_{u u}} d u \\
= & \int_{u_{0, d}(B)}^{u_{0, u}(B)}\left(\frac{R}{u}\right)^{3 / 4} f(u)^{-1 / 2} d u \\
= & -\frac{R^{3 / 4}}{11 u_{0, d}^{2} u_{0, u}^{2} \sqrt{u_{0, d}^{3}-u_{K}^{3}} \sqrt{u_{0, u}^{3}-u_{K}^{3}}} 4\left(u_{0, d} u_{0, u}\right)^{3 / 4} \\
& \times\left\{11 u_{0, d}^{3} u_{0, u}^{5 / 4} \sqrt{u_{0, u}^{3}-u_{K}^{3}}-6 u_{0, u}^{5 / 4} u_{K}^{3} \sqrt{u_{0, u}^{3}-u_{K}^{3}}+u_{0, d}^{5 / 4} \sqrt{u_{0, d}^{3}-u_{K}^{3}}\left(-11 u_{0, u}^{3}+6 u_{K}^{3}\right)\right. \\
+ & \left.6 u_{K}^{3}\left(u_{0, u}^{5 / 4} \sqrt{u_{0, u}^{3}-u_{K}^{3}}{ }_{2} F_{1}\left[-\frac{11}{12}, 1, \frac{7}{12}, \frac{u_{0, d}^{3}}{u_{K}^{3}}\right]-u_{0, d}^{5 / 4} \sqrt{u_{0, d}^{3}-u_{K}^{3}}{ }_{2} F_{1}\left[-\frac{11}{12}, 1, \frac{7}{12}, \frac{u_{0, u}^{3}}{u_{K}^{3}}\right]\right)\right\},
\end{aligned}
$$

with the $B$-dependence of $u_{0, u}$ and $u_{0, d}$ implicit in the last line. Similarly, the same string stretching between $u_{0}$ and $u_{K}$, corresponding to a constituent quark (i.e. this one is a macroscopic string, cfr. the use of the Nambu-Goto action to obtain the expression for the constituent quark mass (3.7)) has a length

$$
\begin{aligned}
L_{q} & =\int d s=\int_{u_{K}}^{u_{0}} \sqrt{g_{u u}} d u \\
& =\int_{u_{K}}^{u_{0}}\left(\frac{R}{u}\right)^{3 / 4} f(u)^{-1 / 2} d u \\
& =R^{3 / 4}\left(-\frac{4 \sqrt{\pi} u_{K}^{1 / 4} \Gamma\left[\frac{11}{12}\right]}{\Gamma\left[\frac{5}{12}\right]}+4 u_{0}^{1 / 4}{ }_{2} F_{1}\left[-\frac{1}{12}, \frac{1}{2}, \frac{11}{12}, \frac{u_{K}^{3}}{u_{0}^{3}}\right]\right) .
\end{aligned}
$$

With our fixed holographic parameters, we have a numerical value for $\ell_{s}$ to compare these lengths to:

$$
\ell_{s}=\sqrt{\alpha^{\prime}} \approx 0.76 \mathrm{GeV}^{-1} .
$$

\footnotetext{
${ }^{10}$ We would like to thank K. Jensen for a private discussion about this.
} 

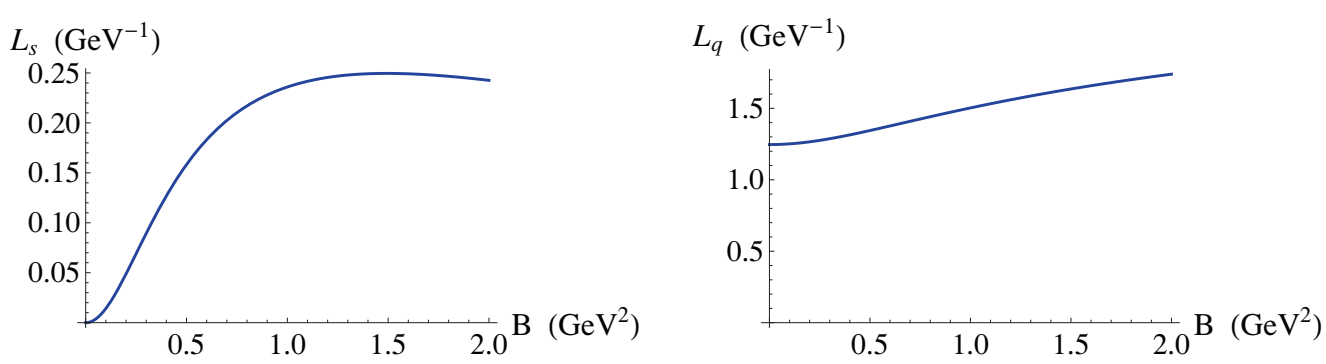

Figure 12. The length $L_{s}(B)$ of a string at $\tau=0$ stretching between $u_{0, d}(B)$ and $u_{0, u}(B)$, and the length $L_{q}(B)$ of a down-quark string stretching between $u_{K}$ and $u_{0, d}(B)$.

From the plots in figure 12 of $L_{s}$ and $L_{q}$ as functions of $B$ up to $2 \mathrm{GeV}^{2}$, we read of estimations of the maximal $L_{s} \approx 0.25 \mathrm{GeV}^{-1}$ and minimal $L_{q} \approx 1.25 \mathrm{GeV}^{-1}$, from which we can conclude that

$$
L_{s}<\ell_{s} \text { and } L_{q}>\ell_{s},
$$

consistent with using the classical Nambu-Goto action for the constituent quark string, but using the non-Abelian DBI-description for the charged rho meson string.

\subsection{Vector sector for full DBI-action}

\subsubsection{Comments on the validity of the $\left(2 \pi \alpha^{\prime}\right)$-expansion}

In the previous section 4.3 and the previous paper [1] we approximated the DBI-action to second order in $\left(2 \pi \alpha^{\prime}\right) F$. The justification that we used for this expansion is roughly that $\alpha^{\prime} \sim 1 / \lambda$ with $\lambda \approx 15$ 'large' in our fixed units. The reader might worry that there is some ambiguity in the proportionality factor $\alpha^{\prime} \sim 1 / \lambda$ since the parameter $X=\lambda \ell_{s}^{2}$ can be chosen freely, as we did in (3.4). The ambiguity should however disappear from all physical quantities and indeed will no longer be present in the full expansion parameter. Let us take a closer look.

Expanding $\operatorname{det}\left(g_{m n}^{D 8}+\left(2 \pi \alpha^{\prime}\right) i F_{m n}\right)=\operatorname{det} g_{m k}^{D 8} \times \operatorname{det}\left(\delta_{n k}+\left(g_{k r}^{D 8}\right)^{-1}\left(2 \pi \alpha^{\prime}\right) i\left(\bar{F}_{r n}+\delta_{1} F_{r n}+\right.\right.$ $\left.\left.\delta_{2} F_{r n}\right)\right)$ in the action $(3.10)$, the expansion parameter $\left(g_{11}^{D 8}\right)^{-1}\left(2 \pi \alpha^{\prime}\right) i \bar{F}_{12}$ is supposed to be small compared to 1 , with (3.15):

$$
\left(\frac{u}{R}\right)^{-3 / 2}\left(2 \pi \alpha^{\prime}\right)\left|\left(\begin{array}{cc}
\frac{2}{3} e B & 0 \\
0 & -\frac{1}{3} e B
\end{array}\right)\right| \ll 1 .
$$

The same expansion parameter can be read off from the form of the matrix A as defined in (3.19). The most strict condition would then be

$$
\left(\frac{u_{0, d}(B=0)}{R}\right)^{-3 / 2}\left(2 \pi \alpha^{\prime}\right) \frac{2}{3} e B \ll 1,
$$

or, in our fixed units,

$$
e B \ll \frac{3}{2}\left(\frac{u_{0, d}(B=0)}{R}\right)^{3 / 2}\left(2 \pi \alpha^{\prime}\right)^{-1} \equiv 0.45 \mathrm{GeV}^{2},
$$


with the appearing combination independent of our choice of $X$ since $u_{0} \sim X, R^{3} \sim X$ and $\left(2 \pi \alpha^{\prime}\right) \sim X$. The instability we found in the $F^{2}$-approximation sets in at $B_{c} \approx 0.8 \mathrm{GeV}^{2}$ (see (4.75)), where the used approximation is thus not necessarily valid anymore. On the other hand, the above is the most strict condition we can impose, it is not so clear what the impact of the $u$-dependence is on this argument. We will therefore use the full STraction and compare with the $F^{2}$-approximation results to provide a conclusive answer to the question of the validity of the $\left(2 \pi \alpha^{\prime}\right)$-expansion in our set-up. It will turn out that using the full STr-action the instability is still present and the value of $B_{c}$ is only slightly higher.

In [71] it is argued that $\alpha^{\prime}$-corrections can cause magnetically induced tachyonic instabilities of $W$-boson strings, stretching between separated D3-branes, to disappear when the inter-brane distance becomes larger than $2 \pi \ell_{s}$. The Landau level spectrum for the $W$-boson is said to receive large $\alpha^{\prime}$-corrections in general [71, 72]. The paper [73] also gives an example where consideration of the full non-Abelian DBI-action in all orders of $\alpha^{\prime}$ - be it using an adapted STr-prescription - can change the physics, that is, the order of the there discussed phase transitions changes.

\subsubsection{Deriving the effective 4-dimensional equations of motion}

Reconsider the vector part of the DBI-Lagrangian in unitary gauge (4.30),

$$
\begin{aligned}
\mathcal{L}= & \mathcal{L}_{\text {Higgs }}+\mathcal{L}_{\text {vector }}=\left.\operatorname{STr} e^{-\phi} \sqrt{-\operatorname{det} a}\right|_{\tilde{A}^{2}} \\
= & \sum_{a=1}^{2}\left\{\gamma(u) \frac{1}{2}\left(\left[\tilde{A}_{u}, \bar{\tau}\right]^{a}\right)^{2}+\alpha(u) \frac{1}{2}\left(\left[\tilde{A}_{\mu}, \bar{\tau}\right]^{a}\right)^{2}+\beta(u) \sum_{\mu=1}^{2} \frac{1}{2}\left(\left[\tilde{A}_{\mu}, \bar{\tau}\right]^{a}\right)^{2}\right\} \\
& +\operatorname{STr} \bar{x}\left\{-\bar{F}_{12} g_{11}^{-2} A^{-1}\left[\tilde{A}_{1}, \tilde{A}_{2}\right]-\left.\frac{1}{4} g_{11}^{-2} \tilde{F}_{\mu \nu}^{2} A^{-2}\right|_{\mu, \nu=1,2}-\left.\frac{1}{2} g_{11}^{-1} G_{u u}^{-1} \tilde{F}_{\mu u}^{2} A^{-1}\right|_{\mu=1,2}\right\}
\end{aligned}
$$

where the notation $\left.\right|_{\mu=1,2}$ as introduced in (4.17) can be written out as

$$
\begin{aligned}
\left.\tilde{F}_{\mu \nu}^{2} A^{-2}\right|_{\mu, \nu=1,2} & =2 A^{-1}\left(\tilde{F}_{i 3}^{2}+\tilde{F}_{i 0}^{2}\right)+2 \tilde{F}_{03}^{2}+2 A^{-2} \tilde{F}_{12}^{2} \quad(i=1,2) \\
& =\tilde{F}_{\mu \nu}^{2}+2 \frac{1-A}{A}\left(\tilde{F}_{i 3}^{2}+\tilde{F}_{i 0}^{2}\right)+2 \frac{1-A^{2}}{A^{2}} \tilde{F}_{12}^{2} \\
\text { and }\left.\quad \tilde{F}_{\mu u}^{2} A^{-1}\right|_{\mu, \nu=1,2} & =\tilde{F}_{\mu u}^{2}+\tilde{F}_{i u}^{2} \frac{1-A}{A} \quad(i=1,2) .
\end{aligned}
$$

Instead of approximating this action further to $\left(2 \pi \alpha^{\prime}\right)^{2} F^{2}$, we now keep all factors of $A=1-\left(2 \pi \alpha^{\prime}\right)^{2} \bar{F}_{12}^{2} \frac{R^{3}}{u^{3}}$. Upon evaluating the STr we then obtain

$$
\begin{aligned}
& \mathcal{L} \sim u^{1 / 4}\left(2 \pi \alpha^{\prime}\right)^{2} \sum_{a, b=1}^{2}\left\{-\left(\sqrt{G_{u u} \bar{F}_{12}} A^{-1 / 2}\right)^{3} \epsilon_{3 a b} \tilde{A}_{1}^{a} \tilde{A}_{2}^{b}-\frac{1}{4} f_{1}\left(\tilde{F}_{\mu \nu}^{a}\right)^{2}-\frac{1}{2} \sum_{i=1}^{2} f_{1 A}\left(\left(\tilde{F}_{i 3}^{a}\right)^{2}+\left(\tilde{F}_{0 i}^{a}\right)^{2}\right)\right. \\
& \left.-\frac{1}{2} f_{1 B}\left(\tilde{F}_{12}^{a}\right)^{2}-\frac{1}{2} g_{11} f_{2}\left(\tilde{F}_{\mu u}^{a}\right)^{2}-\frac{1}{2} g_{11} \sum_{i=1}^{2} f_{2 A}\left(\tilde{F}_{i u}^{a}\right)^{2}-\frac{1}{2} g_{11} \frac{1}{T^{2}} f_{2}\left(\tilde{A}_{\mu}^{a}\right)^{2}\left(2 \bar{\tau}^{3}\right)^{2}-\frac{1}{2} g_{11} \frac{1}{T^{2}} f_{2 A} \sum_{i=1}^{2}\left(\tilde{A}_{i}^{a}\right)^{2}\left(2 \bar{\tau}^{3}\right)^{2}\right\},
\end{aligned}
$$

where we defined the new $I$-functions

$$
\begin{array}{ll}
f_{1}=I\left(G_{u u}^{1 / 2} A^{1 / 2}\right), & f_{1 A}=I\left(\sqrt{G_{u u}} \frac{1-A}{\sqrt{A}}\right), \quad f_{1 B}=I\left(\sqrt{G_{u u}} \sqrt{A} \frac{1-A^{2}}{A^{2}}\right) \\
f_{2}=I\left(G_{u u}^{-1 / 2} A^{1 / 2}\right), & f_{2 A}=I\left(G_{u u}^{-1 / 2} \frac{1-A}{\sqrt{A}}\right),
\end{array}
$$

with $f_{1}$ and $f_{2}$ approaching their previous definition in (4.42) and $f_{1 A}, f_{1 B}$ and $f_{2 A} \rightarrow 0$ for $A \rightarrow 1$ in the $\left(2 \pi \alpha^{\prime}\right)^{2}$-approximation, as they should. 


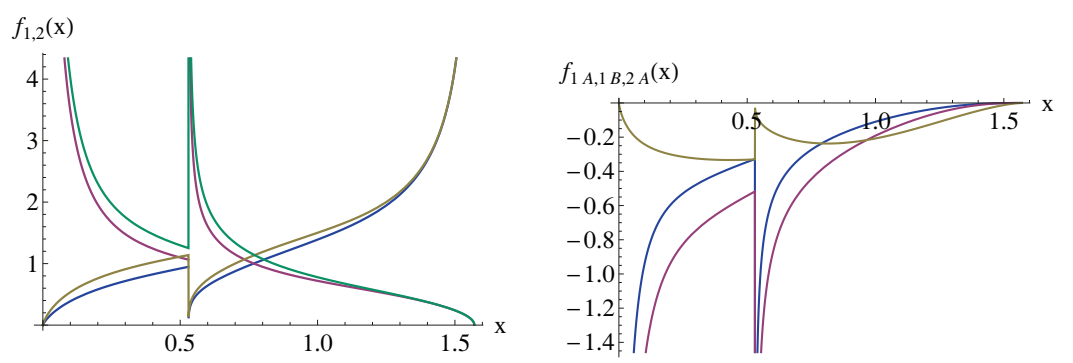

Figure 13. (a) $f_{1}$ (green) and $f_{2}$ (yellow) compared to their $F^{2}$-approximated counterparts in red and blue resp. (b) $f_{1 A}$ (blue), $f_{1 B}$ (red) and $f_{2 A}$ (yellow). For $B=0.8 \mathrm{GeV}^{2}$ and $u=u_{0, d} \cos ^{-3 / 2} x$.

Extracting the effective 4-dimensional action from (4.90) is completely analogous to the procedure described in section 4.3 , so we will give a somewhat more schematic and short explanation here and refer to section 4.3 for more details.

After plugging in the gauge field expansions (4.43)-(4.44) into the action in the approximation of only retaining the lowest modes of the meson towers, one can already notice the vanishing of $\int d u \mathcal{L}_{\text {vector-mixing }}=0$ and of mixing terms between pions and rho mesons. We will focus on the instability in the rho meson sector.

Background dependent functions in the action. The generalized $I$-functions in (4.92) have to be calculated numerically. In figure 13 we compare them to their approximated counterparts for some fixed values of the magnetic field. The measure for the distance between up- and down-brane $\bar{\tau}^{3}(u)$ is still as defined in (4.48), and finally

$$
\left(G_{u u}^{1 / 2} \bar{F}_{12} A^{-1 / 2}\right)^{3}=\sqrt{G_{u u}^{u}} \bar{F}_{u} A_{u}^{-1 / 2}-\sqrt{G_{u u}^{d}} \bar{F}_{d} A_{d}^{-1 / 2}
$$

with $G_{u u}^{l}=G_{u u}\left(\partial_{u} \bar{\tau}^{l}\right)$ (with flavour index $\left.l=u, d\right), \bar{F}_{u}=\frac{2 B}{3}$ and $\bar{F}_{d}=-\frac{B}{3}$ (see (3.15)), and $A_{l}$ defined in (3.19).

Eigenvalue problem. The rho meson part of the DBI-Lagrangian to second order in fluctuations (4.90) after substituting (4.43) reads

$$
\begin{gathered}
\mathcal{L} \sim u^{1 / 4}\left(2 \pi \alpha^{\prime}\right)^{2} \sum_{a, b=1}^{2}\left\{-\frac{1}{4} f_{1}\left(\mathcal{F}_{\mu \nu}^{a}\right)^{2} \psi^{2}-\frac{1}{2} g_{11} f_{2}\left(\rho_{\mu}^{a}\right)^{2}\left(\partial_{u} \psi\right)^{2}-\frac{1}{2} \frac{g_{11}}{\left(2 \pi \alpha^{\prime}\right)^{2}} f_{2}\left(\rho_{\mu}^{a}\right)^{2} \psi^{2}\left(2 \bar{\tau}^{3}\right)^{2}\right. \\
-\frac{1}{2} f_{1 B}\left(F_{12}^{a}\right)^{2}+\sum_{\mu, \nu=1}^{2}\left(-\frac{1}{2} \frac{g_{11}}{\left(2 \pi \alpha^{\prime}\right)^{2}} f_{2 A}\left(\rho_{\mu}^{a}\right)^{2} \psi^{2}\left(2 \bar{\tau}^{3}\right)^{2}-\frac{1}{2}\left(\sqrt{G_{u u}} \bar{F}_{\mu \nu} A^{-1 / 2}\right)^{3} \epsilon_{3 a b} \rho_{\mu}^{a} \rho_{\nu}^{b} \psi^{2}\right. \\
\left.\left.-\frac{1}{2} f_{1 A}\left(\left(\mathcal{F}_{\mu 3}^{a}\right)^{2}+\left(\mathcal{F}_{\mu 0}^{a}\right)^{2}\right) \psi^{2}-\frac{1}{2} g_{11} f_{2 A}\left(\rho_{\mu}^{a}\right)^{2}\left(\partial_{u} \psi\right)^{2}\right)\right\}
\end{gathered}
$$


which results in the following effective 4-dimensional action

$$
\begin{aligned}
S_{4 D} & =\int d^{4} x \sum_{a, b=1}^{2}\left\{-\frac{1}{4}\left(\mathcal{F}_{\mu \nu}^{a}\right)^{2}-\frac{1}{2} m_{\rho}^{2}(B)\left(\rho_{\mu}^{a}\right)^{2}-\frac{1}{2} b(B)\left(\mathcal{F}_{12}^{a}\right)^{2}\right. \\
& \left.+\sum_{\mu, \nu=1}^{2}\left(-\frac{1}{2} a(B)\left(\left(\mathcal{F}_{\mu 3}^{a}\right)^{2}+\left(\mathcal{F}_{\mu 0}^{a}\right)^{2}\right)-\frac{1}{2} m_{+}^{2}(B)\left(\rho_{\mu}^{a}\right)^{2}-\frac{1}{2} \epsilon_{3 a b} \rho_{\mu}^{a} \rho_{\nu}^{b} k(B) \bar{F}_{\mu \nu}^{3}\right)\right\}
\end{aligned}
$$

The function $\psi$ (rescaled to absorb all constant prefactors in the action) satisfies the normalization condition

$$
\int_{u_{0, d}}^{\infty} d u u^{1 / 4} f_{1} \psi^{2}=1
$$

and

$$
\int_{u_{0, d}}^{\infty} d u\left\{u^{1 / 4} g_{11} f_{2} \partial_{u} \psi^{2}+u^{1 / 4} \frac{g_{11}}{\left(2 \pi \alpha^{\prime}\right)^{2}} f_{2}\left(2 \bar{\tau}^{3}\right)^{2} \psi^{2}\right\}=m_{\rho}^{2}
$$

combining into the eigenvalue equation

$$
u^{-1 / 4} f_{1}^{-1} \partial_{u}\left(u^{1 / 4} g_{11} f_{2} \partial_{u} \psi\right)-\frac{g_{11}}{\left(2 \pi \alpha^{\prime}\right)^{2}} f_{1}^{-1} f_{2}\left(2 \bar{\tau}^{3}\right)^{2} \psi=-m_{\rho}^{2} \psi
$$

to be solved for its $B$-dependent eigenvalue $m_{\rho}^{2}$ and eigenfunction $\psi$. The $B$-dependent numbers $m_{+}, k, a$ and $b$ can subsequently be calculated with the obtained eigenfunctions from

$$
\begin{aligned}
\int_{u_{0, d}}^{\infty} d u\left\{u^{1 / 4} g_{11} f_{2 A} \partial_{u} \psi^{2}+u^{1 / 4} \frac{g_{11}}{\left(2 \pi \alpha^{\prime}\right)^{2}} f_{2 A}\left(2 \bar{\tau}^{3}\right)^{2} \psi^{2}\right\} & =m_{+}^{2}, \\
\int_{u_{0, d}}^{\infty} d u u^{1 / 4}\left(\sqrt{G_{u u}} \bar{F}_{12} A^{-1 / 2}\right)^{3} \psi^{2} & =k \bar{F}_{12}^{3}
\end{aligned}
$$

and

$$
\int_{u_{0, d}}^{\infty} d u u^{1 / 4} f_{1 A} \psi^{2}=a, \quad \int_{u_{0, d}}^{\infty} d u u^{1 / 4} f_{1 B} \psi^{2}=b .
$$

The numerical results for $m_{\rho}^{2}, m_{+}^{2}, k, a$ and $b$ as functions of $B$, after having solved the eigenvalue problem with the techniques described in the second paragraph of 4.3.1, are shown in figure 14-15. The discussion of the behaviour of $m_{\rho}^{2}(B)$ in the third paragraph of 4.3.1 is still applicable. The parameter $k$ specifying the strength of the coupling to the magnetic field is again approximately equal to one, but now decreasing as a function of $B$ as opposed to increasing in the $\left(2 \pi \alpha^{\prime}\right)^{2}$-approximation.

\subsubsection{Solving the 4-dimensional equations of motion}

The 4-dimensional EOMs for $\rho_{\nu}^{a}$ derived from the effective action (4.95) are given by $D_{\mu} \mathcal{F}_{\mu \nu}^{a}-\epsilon_{a 3 b} k \bar{F}_{\mu \nu}^{3} \rho_{\mu}^{b}-m_{\rho}^{2} \rho_{\nu}^{a}-\delta_{\nu i}\left(m_{+}^{2} \rho_{i}^{a}+a\left(D_{3} \mathcal{F}_{i 3}^{a}-D_{0} \mathcal{F}_{i 0}^{a}\right)+b D_{j} \mathcal{F}_{i j}^{a}\right)+\delta_{\nu 3} a D_{i} \mathcal{F}_{i 3}^{a}-\delta_{\nu 0} a D_{i} \mathcal{F}_{i 0}^{a}=0$

with $D_{\mu}=\partial_{\mu}+\left[\bar{A}_{\mu}, \cdot\right]$ and $\mathcal{F}_{\mu \nu}^{a}=D_{\mu} \rho_{\nu}^{a}-D_{\nu} \rho_{\mu}^{a}$, and where from now on we will not only keep assuming the Einstein convention that double $\mu, \nu$ indices are Minkowski sums over 

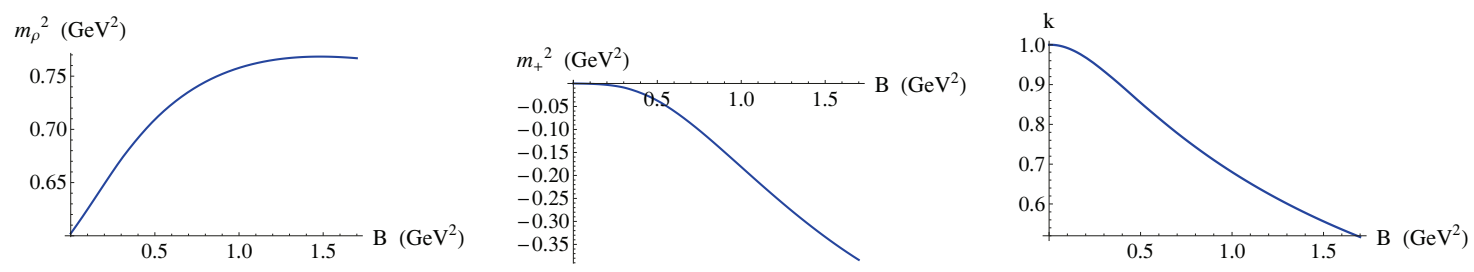

Figure 14. Numerical results for $m_{\rho}^{2}(B), m_{+}^{2}(B)$ and $k(B)$ from the full DBI-action.
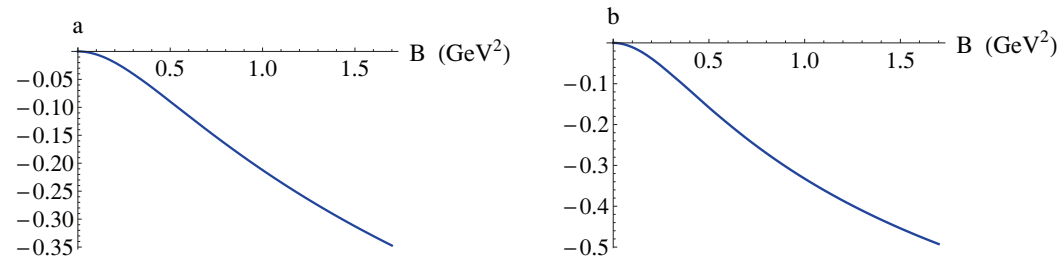

Figure 15. Numerical results for $a(B)$ and $b(B)$.

$\mu, \nu=0 \ldots 3$ but also that double $i, j$ indices are sums over spatial indices $i, j=1,2$. For notational clarity we will not explicitly write out the $B$-dependence of the parameters $m_{\rho}$, $m_{+}, k, a$ and $b$ in this section, but assume it understood.

The equations $(a=1) \pm i(a=2)$ combine into the EOM for the charged rho meson $\rho_{\mu}=\left(\rho_{\mu}^{1}+i \rho_{\mu}^{2}\right) / \sqrt{2}$

$\mathrm{D}_{\mu} \mathrm{F}_{\mu \nu}-i k \bar{F}_{\mu \nu}^{3} \rho_{\mu}-m_{\rho}^{2} \rho_{\nu}-\delta_{\nu i}\left[b \mathrm{D}_{j} \mathrm{~F}_{i j}+a\left(\mathrm{D}_{3} \mathrm{~F}_{i 3}-\mathrm{D}_{0} \mathrm{~F}_{i 0}\right)+m_{+}^{2} \rho_{i}\right]+\delta_{\nu 3} a \mathrm{D}_{j} \mathrm{~F}_{j 3}-\delta_{\nu 0} a \mathrm{D}_{j} \mathrm{~F}_{j 0}=0$,

with $\mathrm{D}_{\mu}=\partial_{\mu}+i \bar{A}_{\mu}^{3}$ and $\mathrm{F}_{\mu \nu}=\mathrm{D}_{\mu} \rho_{\nu}-\mathrm{D}_{\nu} \rho_{\mu}$, and the complex conjugate of this equation for the other charged combination $\rho_{\mu}^{*}=\left(\rho_{\mu}^{1}-i \rho_{\mu}^{2}\right) / \sqrt{2}$. Using $\left[\mathrm{D}_{\mu}, \mathrm{D}_{\nu}\right]=i \bar{F}_{\mu \nu}^{3}$, (4.103) can be rewritten to the following EOMs for resp. $\nu=i$ and $\nu=3$ :

$\underline{\nu}=i$

$$
(1+a) \mathrm{D}_{\mu}^{2} \rho_{\nu}-i(1+b+k) \bar{F}_{\mu \nu}^{3} \rho_{\mu}-(1+a) \mathrm{D}_{\nu} \mathrm{D}_{\mu} \rho_{\mu}-\left(m_{\rho}^{2}+m_{+}^{2}\right) \rho_{\nu}+(b-a)\left(\mathrm{D}_{j}^{2} \rho_{\nu}-\mathrm{D}_{\nu} \mathrm{D}_{j} \rho_{j}\right)=0,
$$

$\underline{\nu}=3$

$$
\mathrm{D}_{\mu}^{2} \rho_{\nu}-\mathrm{D}_{\nu} \mathrm{D}_{\mu} \rho_{\mu}-m_{\rho}^{2} \rho_{\nu}+a\left(\mathrm{D}_{j}^{2} \rho_{\nu}-\mathrm{D}_{\nu} \mathrm{D}_{j} \rho_{j}\right)=0
$$

These equations have to be complemented with a subsidiary condition, obtained by acting with $\mathrm{D}_{\nu}$ on the EOM (4.103) and again using $\left[\mathrm{D}_{\mu}, \mathrm{D}_{\nu}\right]=i \bar{F}_{\mu \nu}^{3}$. We find the generalized subsidiary condition (where by generalized we mean w.r.t. the Proca subsidiary condition $\left.\mathrm{D}_{\nu} \rho_{\nu}=0\right)$

$$
\mathrm{D}_{\nu} \rho_{\nu}=\frac{i}{m_{\rho}^{2}}(1+b-k) \bar{F}_{\mu \nu}^{3} \mathrm{D}_{\nu} \rho_{\mu}-\frac{m_{+}^{2}}{m_{\rho}^{2}} \mathrm{D}_{i} \rho_{i},
$$

still relating $\mathrm{D}_{\nu} \rho_{\nu}(\nu=0 \ldots 3)$ to transversal components $\rho_{i}(i=1,2)$ only, such that the EOMs for the transverse rho mesons can be rewritten as independent from any longitudinal components. Before doing so, let us remark that the above system of EOMs combined with the subsidiary condition reduces to its standard Proca form for $a, b$, 
$m_{+} \rightarrow 0, k \rightarrow 1$ and no $B$-dependence in $m_{\rho}$ (or any of the previous parameters). The non-zero and $B$-dependent $a$ and $b$ are present due to taking into account all powers in the field strength in the non-linear non-Abelian DBI-action, which is also partly the reason for the $B$-dependence of $m_{\rho}, k$ and $m_{+}$, in addition to their implicit description of the response of the quark constituents to the magnetic field (cfr. the chiral magnetic catalysis and holographic Higgs mechanism for heavy-light mesons discussed earlier).

To determine the solutions of the EOMs we follow and generalize the procedure used in [74]. In order to make comparisons with the original expressions in [74] more clear, we temporarily change notation to

$$
\phi_{\mu}=\rho_{\mu}^{*}=\left(\rho_{\mu}^{1}-i \rho_{\mu}^{2}\right) / \sqrt{2}
$$

and

$$
i \pi_{\mu}=\mathrm{D}_{\mu}^{*}=\partial_{\mu}-i \bar{A}_{\mu}^{3}
$$

such that $\pi_{\mu}$ becomes $p_{\mu}-\bar{A}_{\mu}^{3}$ when substituting a plane wave ansatz $\phi_{\mu} \rightarrow e^{i \vec{p} \cdot \vec{x}-i E t} \phi_{\mu}$ into (4.104)-(4.105), and in particular we can write $\pi_{\nu}^{2}=-E^{2}+\vec{\pi}^{2}$. In this new notation the EOMs (4.104)-(4.105) combined with (4.106) can be recast in the form

$E^{2} \phi_{ \pm}=\left(\frac{m_{\rho}^{2}+m_{+}^{2}}{1+a}+\mathcal{B} \vec{\pi}^{2}\right) \phi_{ \pm}+\frac{B}{2 m^{2}}(1+b-k) \pi_{ \pm}\left(\pi_{+} \phi_{-}-\pi_{-} \phi_{+}\right) \pm B \mathcal{K} \phi_{ \pm}-\frac{1}{2} \mathcal{M} \pi_{ \pm}\left(\pi_{+} \phi_{-}+\pi_{-} \phi_{+}\right)$

with

$$
\pi_{ \pm}=\pi_{1} \pm i \pi_{2}, \quad \phi_{ \pm}=\phi_{1} \pm i \phi_{2}
$$

and

$E^{2} \phi_{3}=\left(m_{\rho}^{2}+(1+a) \vec{\pi}^{2}\right) \phi_{3}+\frac{B}{2 m^{2}}(1+b-k) \pi_{3}\left(\pi_{+} \phi_{-}-\pi_{-} \phi_{+}\right)-\frac{1}{2}\left(a-\frac{m_{+}^{2}}{m_{\rho}^{2}}\right) \pi_{3}\left(\pi_{+} \phi_{-}+\pi_{-} \phi_{+}\right)$,

where we defined

$$
\mathcal{B}=\frac{1+b}{1+a}, \quad \mathcal{K}=\frac{1+b+k}{1+a} \quad \text { and } \quad \mathcal{M}=\frac{b-a}{1+a}-\frac{m_{+}^{2}}{m_{\rho}^{2}} .
$$

The main trick for solving the system is to notice that the operators $\pi_{ \pm}$obey the algebra of a simple harmonic oscillator, if one defines annihilation and creation operators $\hat{a}$ and $\hat{a}^{\dagger}$ as

$$
\hat{a}=(2 B)^{-1 / 2} \pi_{+} \quad \text { and } \quad \hat{a}^{\dagger}=(2 B)^{-1 / 2} \pi_{-},
$$

which obey

$$
\left[\hat{a}, \hat{a}^{\dagger}\right]=1 \quad \text { and } \quad\left[\hat{a}, \pi_{3}\right]=\left[\hat{a}^{\dagger}, \pi_{3}\right]=0 .
$$

The 'number operator' $\hat{N}$ is then defined as

$$
\hat{N}=\hat{a}^{\dagger} \hat{a}
$$


allowing us to rewrite the system (4.109)-(4.111), using $\vec{\pi}^{2}=p_{3}^{2}+B(2 \hat{N}+1)$ and $\pi_{+} \pi_{-}=2 B(1+\hat{N})$, to

$$
\left\{\begin{array}{l}
\left(\omega^{2}-\hat{X}_{+}\right) \phi_{+}=A_{\xi} \hat{a}^{2} \phi_{-} \\
\left(\omega^{2}-\hat{X}_{-}\right) \phi_{-}=-B_{\xi}\left(\hat{a}^{\dagger}\right)^{2} \phi_{+} \\
{\left[\omega_{3}^{2}-(1+a)(2 \hat{N}+1) \xi\right] \phi_{3}=\xi^{2}(1+b-k) a_{3}\left(\hat{a} \phi_{-}-\hat{a}^{\dagger} \phi_{+}\right)-\left(a-\frac{m_{+}^{2}}{m_{\rho}^{2}}\right) \xi a_{3}\left(\hat{a} \phi_{-}+\hat{a}^{\dagger} \phi_{+}\right),}
\end{array}\right.
$$

with $\xi=\frac{B}{m_{\rho}^{2}}$ and

$$
\begin{aligned}
\omega^{2} & =\frac{E^{2}-\left(m_{\rho}^{2}+m_{+}^{2}\right) /(1+a)-\mathcal{B} p_{3}^{2}}{m_{\rho}^{2}} \\
\omega_{3}^{2} & =\frac{E^{2}-m_{\rho}^{2}-(1+a) p_{3}^{2}}{m_{\rho}^{2}} \\
\hat{X}_{+} & =(2 \hat{N}+1) \mathcal{B} \xi-\frac{B_{\xi}}{2}+\mathcal{K} \xi-(2 \hat{N}+1) \frac{B_{\xi}}{2} \\
\hat{X}_{-} & =(2 \hat{N}+1) \mathcal{B} \xi-\frac{A_{\xi}}{2}-\mathcal{K} \xi+(2 \hat{N}+1) \frac{A_{\xi}}{2} \\
A_{\xi} & =(1+b-k) \xi^{2}-\mathcal{M} \xi \quad \text { and } \quad B_{\xi}=(1+b-k) \xi^{2}+\mathcal{M} \xi
\end{aligned}
$$

and with $\pi_{3}$ replaced by its eigenvalue $p_{3}$ since it commutes with everything, or where convenient for the notation by the number $a_{3}=(2 B)^{-1 / 2} \pi_{3}$. The system (4.116) decouples completely in the special case where $A_{\xi}=B_{\xi}=0$ as well as $1+b-k=a-\frac{m_{+}^{2}}{m_{\rho}^{2}}=0$, which is for example the case for standard Proca parameters $a=b=m_{+}=0$ and ${ }^{\rho} k=1$. In the latter situation the independent solutions for any $n$ are given by

$$
\begin{aligned}
& \phi_{+}=|n-2\rangle, \quad \phi_{-}=\phi_{3}=0 \quad(n=2,3, \cdots) \\
& \phi_{-}=|n\rangle, \quad \phi_{+}=\phi_{3}=0 \quad(n=0,1, \cdots) \\
& \phi_{3}=|n-1\rangle, \quad \phi_{-}=\phi_{+}=0 \quad(n=1,2, \cdots)
\end{aligned}
$$

with eigenvalue $\omega^{2}=\omega_{3}^{2}=(2 n-1) \xi$. Here we formally defined the 'number eigenstates' $|n\rangle$ as

$$
\hat{N}|n\rangle=n|n\rangle, \quad \hat{a}|0\rangle=0, \quad|n\rangle=(n !)^{-1 / 2}\left(\hat{a}^{\dagger}\right)^{n}|0\rangle .
$$

In the rest of the discussion of possible solutions below, we consider $A_{\xi}$ and $B_{\xi}$ different from zero.

Condensing solution. Before decoupling the first two equations of (4.116) to discuss the general form of the solution, let us first look at the one we are most interested in, the condensing solution:

$$
\phi_{3}=\phi_{+}=0, \quad \phi_{-}=|0\rangle \quad\left(\Rightarrow \hat{a} \phi_{-}=0\right),
$$

for which the EOM reduces to

$$
\left(\omega^{2}-\hat{X}_{-}\right)|0\rangle=0 \Rightarrow \omega^{2}=\hat{X}_{-}(\hat{N} \rightarrow 0)=(\mathcal{B}-\mathcal{K}) \xi=-\frac{k}{1+a} \xi
$$


with total eigenvalue

$$
E^{2}=\frac{m_{\rho}^{2}+m_{+}^{2}}{1+a}+\left(\frac{1+b}{1+a}\right) p_{3}^{2}-\frac{k}{1+a} m_{\rho}^{2} \xi
$$

or, in the lowest state $p_{3}=0$ (and $\frac{1+b}{1+a}>0$ in the considered range of $B$ ):

$$
m_{\rho, e f f}^{2}=\frac{m_{\rho}^{2}+m_{+}^{2}}{1+a}-\frac{k}{1+a} m_{\rho}^{2} \xi
$$

This indeed reduces to its $\left(2 \pi \alpha^{\prime}\right)^{2}$-approximated equivalent $(4.74), m_{\rho, \text { eff }}^{2}=m_{\rho}^{2}+m_{+}^{2}-$ $k \xi m_{\rho}^{2}$, for $a \rightarrow 0$.

Family of solutions. We present the general discussion of the family of solutions of (4.116). One family of solutions is

$$
\phi_{+}=\phi_{-}=0, \quad \phi_{3}=|n\rangle, \quad \omega_{3}^{2}=(1+a)(2 n+1) \xi, \quad n=0,1,2, \cdots,
$$

the other one

$$
\phi_{-}=|n+1\rangle, \quad \phi_{+}=c_{n}|n-1\rangle, \quad \phi_{3}=c_{n}^{\prime}|n\rangle, \quad n=1,2,3, \cdots .
$$

The corresponding eigenvalue $\omega$ can be determined from decoupling the first two equations of (4.116) to

$$
\begin{aligned}
\left\{\left(\omega^{2}-\hat{X}_{-}\right)\left(\omega^{2}-\hat{X}_{+}\right)+\left(\hat{N}^{2}+3 \hat{N}+2\right) A_{\xi} B_{\xi}-2\left(2 \mathcal{B} \xi+A_{\xi}\right)\left(\omega^{2}-\hat{X}_{+}\right)\right\} \phi_{+} & =0 \\
\left\{\left(\omega^{2}-\hat{X}_{-}\right)\left(\omega^{2}-\hat{X}_{+}\right)+\left(\hat{N}^{2}-\hat{N}\right) A_{\xi} B_{\xi}+2\left(2 \mathcal{B} \xi-B_{\xi}\right)\left(\omega^{2}-\hat{X}_{-}\right)\right\} \phi_{-} & =0 .
\end{aligned}
$$

Substitution of (4.128) has the effect of replacing $\hat{N}$ in (4.129) by $(n-1)$ and in (4.130) by $(n+1)$. With these replacements, the curly-bracketed expressions in the two equations become identical, and either of them can be solved, with the result for our generalized Landau levels finally given by

$$
\begin{aligned}
& \omega^{2}=(2 n+1) \xi\left(\mathcal{B}-\frac{\mathcal{M}}{2}\right)+\frac{(1+b-k)}{2} \xi^{2} \\
& \pm \xi \sqrt{\mathcal{M}\left(\frac{(2 n+1)^{2}}{4}+\mathcal{K}-2 \mathcal{B}\right)+(\mathcal{K}-2 \mathcal{B})^{2}-(1+b-k)(2 n+1) \xi\left(\mathcal{K}-2 \mathcal{B}+\frac{\mathcal{M}}{2}\right)+\frac{(1+b-k)^{2}}{4} \xi^{2}}
\end{aligned}
$$

This reduces to Mathews' solution for general $k \neq 1$, eq. (19) in [74], for $a, b, m_{+} \rightarrow 0$, i.e. $\mathcal{B} \rightarrow 1, \mathcal{M} \rightarrow 0, \mathcal{K} \rightarrow 1+k$ :

$$
\omega^{2}\left(a, b, m_{+} \rightarrow 0\right)=(2 n+1) \xi+\frac{1}{2}(1-k) \xi^{2} \pm(1-k) \xi \sqrt{1+(2 n+1) \xi+\frac{1}{4} \xi^{2}},
$$

and the modified Landau levels mentioned in section 4.3.1 are given by (4.131) with $a, b \rightarrow$ 0 . Given the value of $E^{2}$ from (4.131) and the ansatz (4.128) for $\phi_{3}$, the equation (4.118) can be solved for $c_{n}^{\prime}$. The constant $c_{n}$ can be determined from substituting the solution (4.128) and (4.131) into either one of the first two equations of (4.116).

For completeness, we mention the last remaining possible solution

$$
\phi_{-}=|1\rangle, \quad \phi_{+}=0, \quad \phi_{3}=c_{0}^{\prime}|0\rangle
$$




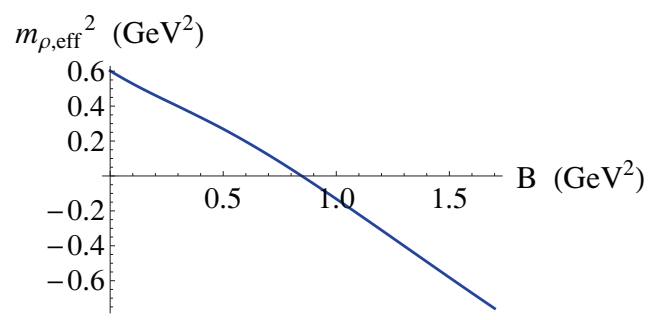

Figure 16. The effective rho meson mass squared $m_{\rho, \text { eff }}^{2}(B)$ from the full DBI-action.

with $\omega^{2}=\hat{X}_{-}(\hat{N} \rightarrow 1)=(3 \mathcal{B}-\mathcal{K}-\mathcal{M}) \xi+(1+b-k) \xi^{2}$ and $c_{0}^{\prime}$ to be determined from $\omega_{3}^{2}-(1+a) \xi c_{0}^{\prime}=\left(\xi^{2}(1+b-k)-a \xi\right) a_{3}$.

In this whole discussion of the solutions of the EOMs for the rho meson, the key observation is that the energy eigenstates are so-called 'number eigenstates', labeled by the Landau level number $n$. They are not necessarily spin eigenstates, as we will discuss next.

Discussion of the spin of the solutions. Consider the eigenstates of the spin operator $S_{3}$ as defined in (4.71),

$$
\begin{array}{ll}
\phi_{+}=\phi_{-}=0 & \left(s_{3}=0\right) \\
\phi_{+}=\phi_{3}=0 & \left(s_{3}=+1\right) \\
\phi_{-}=\phi_{3}=0 & \left(s_{3}=-1\right) .
\end{array}
$$

It is clear that only the branch of solutions (4.127) and the condensing solution (4.124) are spin eigenstates, resp. with eigenvalues $s_{3}=0$ and $s_{3}=+1$; the other branches of solutions for general $k \neq 1$ case are not. This is in contrast with the special $k=1$ Proca case (4.122) where all Landau levels, including the excited states, are also spin eigenstates.

We conclude by summarizing that the condensing states are given by (4.124) and its conjugate,

$$
\rho^{*}=\phi_{-}=\rho_{1}^{*}-i \rho_{2}^{*} \quad \text { and } \quad \rho=\phi_{-}^{*}=\rho_{1}+i \rho_{2}
$$

- where we translated back to the previously used notation - with energy eigenvalue $m_{\rho, \text { eff }}^{2}=(4.126)$ and spin eigenvalue $s_{3}=+1$ corresponding to the spins being aligned with the magnetic field. Our result for the effective rho meson mass squared $m_{\rho, \text { eff }}^{2}$, as shown in figure 16, again demonstrates the tachyonic instability, with the critical magnetic field for rho meson condensation given this time by

$$
B_{c} \approx 0.85 \mathrm{GeV}^{2} .
$$

The increase compared to the estimate for $B_{c}$ in $(4.75)$ using the $\left(2 \pi \alpha^{\prime}\right)^{2}$-approximation is pretty small. This indicates that the expansion to second order in $\left(2 \pi \alpha^{\prime}\right) F$ was a valid approximation, despite the ambiguities mentioned in section 4.4.1.

\subsection{Comment on the antipodal case}

For completeness, we consider the effect in the antipodal SSM, $u_{0}=u_{K}$, of including all higher order terms in the total field strength in the DBI-action. As mentioned before, the 


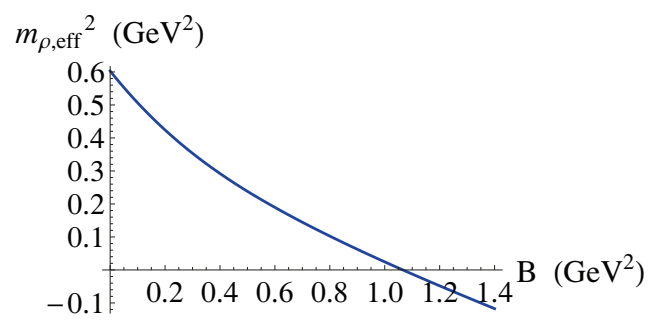

Figure 17. The effective rho meson mass squared $m_{\rho, \text { eff }}^{2}(B)$ from the full DBI-action for the antipodal embedded flavour branes.

embedding of the flavour branes is independent of $B$ in this case, resulting in standard Landau levels and thus $m_{\rho, \text { eff }}^{2}(B)=m_{\rho}^{2}-B$ if the action is approximated to second order in $\left(2 \pi \alpha^{\prime}\right) F$. In this set-up there is no constituent quark mass (3.7) and no chiral magnetic catalysis.

To reproduce $m_{\rho}^{2}=0.602 \mathrm{GeV}^{2}$ at zero magnetic field, along with $f_{\pi}=0.093 \mathrm{GeV}$ for the pion decay constant, we have to use the holographic parameters fixed in [3] to

$$
M_{K} \approx 0.949 \mathrm{GeV} \text { and } \kappa=\frac{\lambda N_{c}}{216 \pi^{3}} \approx 0.00745
$$

instead of the values (3.9) for $u_{0}>u_{K}$. With these fixed parameters the estimate for the maximum value of the magnetic field for the $\left(2 \pi \alpha^{\prime}\right)$-expansion of the action to be valid, as discussed in section 4.4.1, changes to

$$
e B \ll \frac{3}{2}\left(\frac{u_{K}}{R}\right)^{3 / 2}\left(2 \pi \alpha^{\prime}\right)^{-1} \equiv 0.31 \mathrm{GeV}^{2},
$$

which is even lower than the value $0.45 \mathrm{GeV}^{2}$ obtained for the non-antipodal case.

As the flavour branes now remain coincident for any value of $B$, that is $\bar{\tau} \sim \mathbb{1} \Rightarrow \bar{\tau}^{3}=0$ and $\partial_{u} \bar{\tau}=0 \Rightarrow G_{u u}=g_{u u}$, we again obtain the effective 4-dimensional action (4.95), but with the integrals and equations (4.96)-(4.101) changed in the sense that $u_{0, d} \rightarrow u_{K}$, $\bar{\tau}^{3} \rightarrow 0$ and every $G_{u u} \rightarrow g_{u u}$, in particular in the $I$-functions $f_{1(A, B)}, f_{2(A)}$ defined in (4.91)-(4.92). The eigenvalue equation can be recast in the form

$$
\frac{9}{4} \frac{u_{K}}{R^{3}} \cos ^{4 / 3} x\left[\partial_{x}^{2} \psi+I\left(A^{1 / 2}\right)^{-1} \partial_{x} I\left(A^{1 / 2}\right) \partial_{x} \psi\right]=-m_{\rho}^{2} \psi
$$

with $u=u_{K} \cos ^{-2 / 3} x$ this time and $I\left(A^{1 / 2}\right)$ reducing to 1 for $B=0$. With the numerical result for the eigenfunction $\psi$ and eigenvalue $m_{\rho}^{2}$, the total effective rho meson squared can be obtained using (4.126),

$$
m_{\rho, e f f}^{2}=\frac{m_{\rho}^{2}+m_{+}^{2}}{1+a}-\frac{k}{1+a} B .
$$

The result is shown in figure 17 , where the corresponding critical magnetic field can be read off to be $B_{c} \approx 1.07 \mathrm{GeV}^{2}$. 

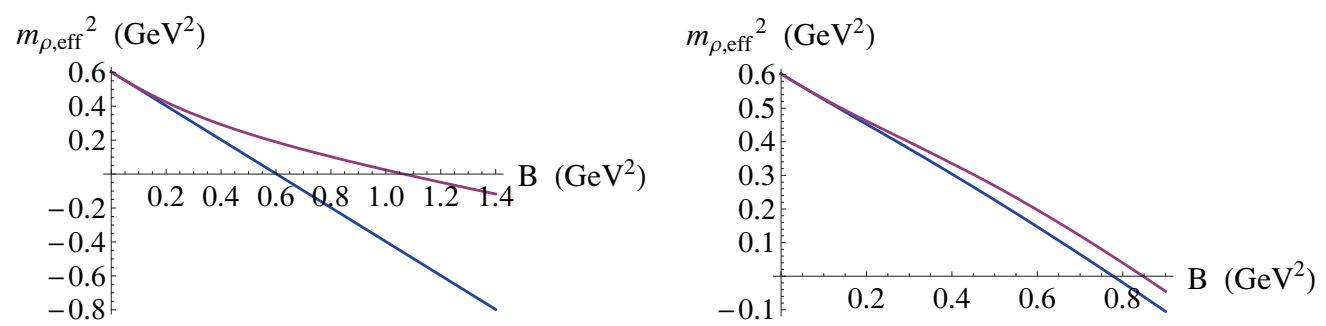

Figure 18. The effective rho meson mass squared $m_{\rho, \text { eff }}^{2}(B)$ in the antipodal embedding (left) and the non-antipodal embedding (right), comparing the $\left(2 \pi \alpha^{\prime}\right)^{2} F^{2}$-approximated result in blue to the full DBI-result in red.

\section{Summary}

We studied a magnetically induced tachyonic instability in the charged rho meson sector, arising from the DBI-part of the two-flavour Sakai-Sugimoto model. We examined both the case of antipodal and the more general non-antipodal embedding, each in the $\left(2 \pi \alpha^{\prime}\right)^{2} F^{2}$-approximation of the action versus the full DBI-action, non-linear in the total field strength $F$. The results for the effective rho meson mass squared $m_{\rho, \text { eff }}^{2}(B)$, vanishing at the critical magnetic field $B_{c}$ and thereby signaling the onset of the tachyonic instability, are shown in figure 18 for each of the four set-ups.

The antipodal SSM reproduces exactly the standard 4-dimensional Proca picture and Landau levels of the effective QCD-model used in [22], with $B_{c}=m_{\rho}^{2} \approx 0.602 \mathrm{GeV}^{2}$. The same picture was obtained in a holographic toy model involving an SU(2) EinsteinYang-Mills action for an SU(2) bulk gauge field in a (4+1)-dimensional AdS-Schwarzschild black hole background [36], and more recently for a 3-dimensional field theory in a (3+1)dimensional DSGS-model generalized to AdS [38]. The non-antipodal SSM predicts a larger value of $B_{c} \approx 0.78 \mathrm{GeV}^{2}$ as a result of taking two mass-generating effects for the charged rho meson into account, i.e. chiral magnetic catalysis for the rho meson constituents on one hand, and a stringy Higgs-contribution to the mass from stretching the rho meson string between the magnetically separated up- and down-brane. Both effects are a direct result from the $B$-dependence of the non-antipodal flavour branes' embedding, and hence absent in the antipodal set-up. Considering the full DBI-action instead of approximating it to second order in the total field strength further increases the value of the magnetic field $B_{c}$ at the onset of rho meson condensation, more precisely to $B_{c} \approx 0.85 \mathrm{GeV}^{2}$ in the non-antipodal case. The effect of taking the non-linear contributions in $\bar{F}_{12}$ into account seems to be stronger for the antipodal set of parameters compared to the non-antipodal one - in both cases parameters are fixed to reproduce QCD parameters at zero magnetic field. This leads us to conclude that the $F^{2}$-approximation is better justified for the considered problem in the nonantipodal embedding than in the antipodal one. We are however very well aware of the fact that the full DBI-action is not the complete non-Abelian action for a system of $N_{f}$ branes a closed form of which is still to be found -, starting to show deviations at order $F^{6}[59,60]$. We do not claim the DBI-result is necessarily more correct than the $F^{2}$-result, yet we wanted to examine the extent of the difference. In conclusion, the SSM-predictions for $B_{c}$ are close to order $1 \mathrm{GeV}^{2}$, as obtained in the NJL-model in [23] and on the lattice in [33]. 
A main motivation for these comparisons within the SSM was to investigate what holography can add to the QCD-phenomenological picture of rho meson condensation, purposely working in a top-down approach - the downside of which are the technical complications. We for example elaborated on evaluating the STr exactly (to second order in fluctuations in the presence of an Abelian background field), the gauge fixing necessary to disentangle scalar and vector fluctuations, the contribution of the Chern-Simons action, the pion sector in the $F^{2}$-approximated DBI-part of the action, the Higgs mechanism associated with the magnetically induced heavy-light character of the charged rho mesons, numerically solving the eigenvalue equation for $m_{\rho}^{2}$ with a shooting method, and analytically solving the generalized effective 4-dimensional EOMs. For the above reasons of complexity we have not yet been able to construct the new ground state in which the rho mesons are condensed. This ground state is expected to be an Abrikosov lattice of rho meson vortices, as constructed in the DSGS-model in [34] and in a bottom-up holographic model in [37]. The Abrikosov lattice forms an anisotropic and spatially inhomogeneous, type II superconducting ground state of the QCD vacuum in the presence of a strong magnetic field [35], with the interesting property that the magnetic field creates the superconducting state instead of destroying it (cfr. Meissner effect). In [38], the real part of the optical conductivity in the condensed phase is shown to contain a delta peak at the origin, consistent with a superconducting condensed state. Another downside of the top-down approach and in particular the SSM is the abundance of extra fields in the bulk that do not have counterparts in the dual field theory. The mass scale $\sim M_{K}$ of these artifacts of the model is actually of the same order as the masses of the mesons. Nevertheless the SSM can present a nice record of QCD-effects and properties that can be modeled, suggesting the influence of the redundant modes is not necessarily substantial.

We have been able to show that the SSM has a magnetically induced instability towards rho meson condensation, consistent with the studies of this phenomenon in phenomenological [22, 23], lattice [33] and bottom-up holographic [36, 38] approaches. To come closer to the real-life quark-gluon plasma conditions where the presence of magnetic fields of the order of $\sim 1 \mathrm{GeV}^{2}$ might eventually be obtained, it should be taken into account that there are also very high temperatures/densities present, and that the magnetic field is very localized both in space and time (see the more recent works cited under [24-29]). These features may in the end seriously influence the possible occurrence of rho meson condensation.

\section{Acknowledgments}

It is a pleasure to thank A. Sevrin for helpful discussions. N. C. is supported in part by the Belgian Federal Science Policy Office through the Interuniversity Attraction Pole P7/37, by FWO-Vlaanderen through the project G.0114.10N, and by the Vrije Universiteit Brussel through the Strategic Research Program "High-Energy Physics". N. C. and D. D. are supported by FWO-Vlaanderen. 


\section{A STr-prescription}

Prescription. We write down the prescription for the evaluation of the symmetrized trace STr to second order in fluctuations in the presence of a constant Abelian background, as derived in [59] and [63].

For an even function $\mathcal{H}(F)$ of a diagonal background field $F=F^{0} \sigma^{0}+F^{3} \sigma^{3}$ and fluctuation $\tilde{X}=\tilde{X}^{a} t^{a}$ (generator $t^{a}=-\frac{i}{2} \sigma^{a}$ ), one finds that

$$
\operatorname{STr}\left(\mathcal{H}(F) \tilde{X}^{2}\right)=-\frac{1}{2} \sum_{a=1}^{2}\left(\tilde{X}^{a}\right)^{2} I(\mathcal{H})-\frac{1}{2} \sum_{l=u, d}\left(\tilde{X}^{l}\right)^{2} I_{l}(\mathcal{H})
$$

with

$$
\begin{array}{rlrl}
I(\mathcal{H}) & =\frac{\int_{0}^{1} d \alpha \mathcal{H}\left(F^{0}+\alpha F^{3}\right)+\int_{0}^{1} d \alpha \mathcal{H}\left(F^{0}-\alpha F^{3}\right)}{2}, \\
I_{u}(\mathcal{H}) & =\mathcal{H}\left(F^{0}+F^{3}\right), & I_{d}(\mathcal{H})=\mathcal{H}\left(F^{0}-F^{3}\right), \\
\tilde{X}^{u} & =\frac{\tilde{X}^{0}+\tilde{X}^{3}}{\sqrt{2}}, & \tilde{X}^{d}=\frac{\tilde{X}^{0}-\tilde{X}^{3}}{\sqrt{2}}
\end{array}
$$

and

$$
\mathrm{S} \operatorname{Tr}(\mathcal{H}(F) \tilde{X})=\operatorname{Tr}(\mathcal{H}(F) \tilde{X}) .
$$

Generalized prescription. A straightforward generalization of the prescription when dealing with two Abelian background fields can be written down.

For even functions $\mathcal{H}(\partial \bar{\tau})$ and $\mathcal{G}(F)$ of diagonal background fields $\partial \bar{\tau}=\partial \bar{\tau}^{0} \sigma^{0}+\partial \bar{\tau}^{3} \sigma^{3}$ and $F=F^{0} \sigma^{0}+F^{3} \sigma^{3}$, and fluctuation $\tilde{X}=\tilde{X}^{a} t^{a}$ (generator $t^{a}=-\frac{i}{2} \sigma^{a}$ ), it reads

$$
\operatorname{STr}\left(\mathcal{H}(\partial \bar{\tau}) \mathcal{G}(F) \tilde{X}^{2}\right)=-\frac{1}{2} \sum_{a=1}^{2}\left(\tilde{X}^{a}\right)^{2} I(\mathcal{H G})-\frac{1}{2} \sum_{l=u, d}\left(\tilde{X}^{l}\right)^{2} I_{l}(\mathcal{H G})
$$

with

$$
\begin{array}{rlrl}
I(\mathcal{H}) & =\frac{\int_{0}^{1} d \alpha \mathcal{H}\left(\partial \bar{\tau}^{0}+\alpha \partial \bar{\tau}^{3}\right) \mathcal{G}\left(F^{0}+\alpha F^{3}\right)+\int_{0}^{1} d \alpha \mathcal{H}\left(\partial \bar{\tau}^{0}-\alpha \partial \bar{\tau}^{3}\right) \mathcal{G}\left(F^{0}-\alpha F^{3}\right)}{2}, \\
I_{u}(\mathcal{H} \mathcal{G}) & =\mathcal{H}\left(\partial \bar{\tau}^{0}+\partial \bar{\tau}^{3}\right) \mathcal{G}\left(F^{0}+F^{3}\right), & I_{d}(\mathcal{H} \mathcal{G})=\mathcal{H}\left(\partial \bar{\tau}^{0}-\partial \bar{\tau}^{3}\right) \mathcal{G}\left(F^{0}-F^{3}\right), \\
\tilde{X}^{u} & =\frac{\tilde{X}^{0}+\tilde{X}^{3}}{\sqrt{2}}, & \tilde{X}^{d}=\frac{\tilde{X}^{0}-\tilde{X}^{3}}{\sqrt{2}}
\end{array}
$$

and

$$
\mathrm{S} \operatorname{Tr}(\mathcal{H}(\partial \bar{\tau}) \mathcal{G}(F) \tilde{X})=\operatorname{Tr}(\mathcal{H}(\partial \bar{\tau}) \mathcal{G}(F) \tilde{X}) .
$$

\section{A.1 Derivation of the prescription}

For completeness, let us schematically recapitulate how the above prescription was obtained. In this derivation we will temporarily write $\mathrm{U}(2)$-indices as lower instead of upper indices, to avoid notational clutter. 
- Properties of the Pauli matrices $(a=1,2,3)$ :

$$
\begin{aligned}
& \operatorname{Tr}\left(\sigma_{a}\right)=0, \quad \operatorname{Tr}\left(\sigma_{a} \sigma_{b}\right)=2 \delta_{a b}, \quad \sigma_{a} \sigma_{b}=\delta_{a b} \mathbb{1}+i \epsilon_{a b c} \sigma_{c} \\
& \left\{\sigma_{a}, \sigma_{b}\right\}=2 \delta_{a b} \mathbb{1}, \quad\left[\sigma_{a}, \sigma_{b}\right]=2 i \epsilon_{a b c} \sigma_{c}
\end{aligned}
$$

- $\operatorname{STr}\left(\sigma_{3}^{m} \sigma_{a} \sigma_{b}\right)$ :

$$
\begin{aligned}
\operatorname{STr}\left(\sigma_{3}^{m} \sigma_{a} \sigma_{b}\right) & =\frac{1}{(m+2) !} \sum_{\text {all permutations }} \operatorname{Tr}\left(\sigma_{3}^{m} \sigma_{a} \sigma_{b}\right) \\
& =\frac{1}{m+1} \sum_{k=0}^{m} \operatorname{Tr}\left(\sigma_{3}^{k} \sigma_{a} \sigma_{3}^{m-k} \sigma_{b}\right) \\
& =\left\{\begin{array}{ll}
2\left[\delta_{0 a} \delta_{0 b}+\delta_{3 a} \delta_{3 b}+\left.\frac{\delta_{a b}}{m+1}\right|_{a, b=1,2}\right] \\
2\left[\delta_{0 a} \delta_{3 b}+\delta_{3 a} \delta_{0 b}\right] & \text { for } m \text { odd }
\end{array} \text { for } m\right. \text { even }
\end{aligned}
$$

where now $a, b=0,1,2,3$ with $\sigma_{0}=\mathbb{1}$, and where we used

$$
\sum_{k=0}^{m} \operatorname{Tr}\left(\sigma_{3}^{k} \sigma_{a} \sigma_{3}^{m-k} \sigma_{b}\right)=\sum_{k=0}^{m} \operatorname{Tr}\left((-1)^{k} \sigma_{3}^{m} \sigma_{b} \sigma_{a}\right) .
$$

- $\operatorname{STr}\left(F^{m} \tilde{X}^{2}\right)$ with $m$ even, $F=F_{0} \sigma_{0}+F_{3} \sigma_{3}$ and $\tilde{X}=\tilde{X}_{a} t_{a}$ with $t_{a}=-i\left(\frac{\mathbb{1}}{2}, \frac{\sigma_{a}}{2}\right)$ :

$$
\begin{aligned}
\operatorname{STr}\left(F^{m} \tilde{X}^{2}\right)= & F_{3}^{m} \operatorname{STr}\left(\sigma_{3}^{m} \tilde{X}^{2}\right)+F_{3}^{m-1} F_{0}\left(\begin{array}{c}
m \\
1
\end{array}\right) \operatorname{STr}\left(\sigma_{3}^{m-1} \tilde{X}^{2}\right)+F_{3}^{m-2} F_{0}^{2}\left(\begin{array}{c}
m \\
2
\end{array}\right) \operatorname{STr}\left(\sigma_{3}^{m-2} \tilde{X}^{2}\right) \\
& +\cdots+F_{0}^{m} \operatorname{STr}\left(\tilde{X}^{2}\right) \\
= & -\frac{1}{2} F_{3}^{m}\left[\tilde{X}_{0}^{2}+\tilde{X}_{3}^{2}+\sum_{a=1}^{2} \frac{\tilde{X}_{a}^{2}}{m+1}\right]-\frac{1}{2} F_{3}^{m-1} F_{0}\left(\begin{array}{c}
m \\
1
\end{array}\right)\left[\tilde{X}_{0} \tilde{X}_{3}+\tilde{X}_{3} \tilde{X}_{0}\right] \\
& -\frac{1}{2} F_{3}^{m-2} F_{0}^{2}\left(\begin{array}{c}
m \\
2
\end{array}\right)\left[\tilde{X}_{0}^{2}+\tilde{X}_{3}^{2}+\sum_{a=1}^{2} \frac{\tilde{X}_{a}^{2}}{m-1}\right]+\cdots-\frac{1}{2} F_{0}^{m} \sum_{a=0}^{3} \tilde{X}_{a}^{2} \\
= & -\frac{1}{2} \sum_{a=1}^{2} \tilde{X}_{a}^{2}\left\{\frac{F_{3}^{m}}{m+1}+\frac{F_{3}^{m-2} F_{0}^{2}}{m-1}\left(\begin{array}{c}
m \\
2
\end{array}\right)+\cdots+\frac{F_{3}^{2} F_{0}^{m-2}}{3}\left(\begin{array}{c}
m \\
2
\end{array}\right)+F_{0}^{m}\right\} \\
& -\frac{1}{2}\left(\tilde{X}_{0}^{2}+\tilde{X}_{3}^{2}\right)\left\{F_{3}^{m}+F_{3}^{m-2} F_{0}^{2}\left(\begin{array}{c}
m \\
2
\end{array}\right)+\cdots+F_{0}^{m}\right\} \\
& -\frac{1}{2}\left(2 \tilde{X}_{0} \tilde{X}_{3}\right)\left\{F_{3}^{m-1} F_{0}\left(\begin{array}{c}
m \\
1
\end{array}\right)+F_{3}^{m-3} F_{0}^{3}\left(\begin{array}{c}
m \\
3
\end{array}\right)+\cdots+F_{3} F_{0}^{m-1}\left(\begin{array}{c}
m \\
1
\end{array}\right)\right\}
\end{aligned}
$$

- $\operatorname{STr}\left(\mathcal{H}(F) X^{2}\right)$ with $\mathcal{H}(F)=a_{0}+a_{1} F^{2}+a_{2} F^{4}+\cdots+a_{m} F^{2 m}+\cdots$ an even function of the background field $F$ :

$$
\begin{aligned}
\operatorname{STr}\left(\mathcal{H}(F) \tilde{X}^{2}\right)= & -\frac{1}{2} \sum_{a=1}^{2} \tilde{X}_{a}^{2}\left\{a_{0}+a_{1}\left[\frac{F_{3}^{2}}{3}+F_{0}^{2}\right]+a_{2}\left[\frac{F_{3}^{4}}{5}+\left(\begin{array}{l}
4 \\
2
\end{array}\right) \frac{F_{3}^{2} F_{0}^{2}}{3}+F_{0}^{4}\right]+\cdots\right\} \\
& -\frac{1}{2}\left(\tilde{X}_{0}^{2}+\tilde{X}_{3}^{2}\right)\left\{a_{0}+a_{1}\left[F_{3}^{2}+F_{0}^{2}\right]+a_{2}\left[F_{3}^{4}+\left(\begin{array}{l}
4 \\
2
\end{array}\right) F_{3}^{2} F_{0}^{2}+F_{0}^{4}\right]+\cdots\right\} \\
& -\frac{1}{2}\left(2 \tilde{X}_{0} \tilde{X}_{3}\right)\left\{a_{1}\left[\left(\begin{array}{l}
2 \\
1
\end{array}\right) F_{0} F_{3}\right]+a_{2}\left[\left(\begin{array}{l}
4 \\
1
\end{array}\right) F_{0}^{3} F_{3}+\left(\begin{array}{l}
4 \\
1
\end{array}\right) F_{0} F_{3}^{3}\right]+\cdots\right\}
\end{aligned}
$$




$$
\begin{aligned}
= & -\frac{1}{2} \sum_{a=1}^{2} \tilde{X}_{a}^{2}\left\{\frac{\int_{0}^{1} d \alpha \mathcal{H}\left(F_{0}+\alpha F_{3}\right)+\int_{0}^{1} d \alpha \mathcal{H}\left(F_{0}-\alpha F_{3}\right)}{2}\right\} \\
& -\frac{1}{2}\left(\tilde{X}_{0}^{2}+\tilde{X}_{3}^{2}\right)\left\{\frac{\mathcal{H}\left(F_{0}+F_{3}\right)+\mathcal{H}\left(F_{0}-F_{3}\right)}{2}\right\} \\
& -\frac{1}{2}\left(2 \tilde{X}_{0} \tilde{X}_{3}\right)\left\{\frac{\mathcal{H}\left(F_{0}+F_{3}\right)-\mathcal{H}\left(F_{0}-F_{3}\right)}{2}\right\}
\end{aligned}
$$

which is the prescription (A.1).

Open Access. This article is distributed under the terms of the Creative Commons Attribution License (CC-BY 4.0), which permits any use, distribution and reproduction in any medium, provided the original author(s) and source are credited.

\section{References}

[1] N. Callebaut, D. Dudal and H. Verschelde, Holographic $\rho$ mesons in an external magnetic field, JHEP 03 (2013) 033 [arXiv: 1105.2217] [INSPIRE].

[2] T. Sakai and S. Sugimoto, Low energy hadron physics in holographic QCD, Prog. Theor. Phys. 113 (2005) 843 [hep-th/0412141] [INSPIRE].

[3] T. Sakai and S. Sugimoto, More on a holographic dual of QCD, Prog. Theor. Phys. 114 (2005) 1083 [hep-th/0507073] [INSPIRE].

[4] H. Ooguri and C.-S. Park, Spatially modulated phase in holographic quark-gluon plasma, Phys. Rev. Lett. 106 (2011) 061601 [arXiv: 1011.4144] [INSPIRE].

[5] K. Fukushima and P. Morales, Spatial modulation and topological current in holographic QCD matter, Phys. Rev. Lett. 111 (2013) 051601 [arXiv:1305.4115] [INSPIRE].

[6] J. de Boer, B.D. Chowdhury, M.P. Heller and J. Jankowski, Towards a holographic realization of the quarkyonic phase, Phys. Rev. D 87 (2013) 066009 [arXiv:1209.5915] [InSPIRE].

[7] C.B. Bayona, K. Peeters and M. Zamaklar, A non-homogeneous ground state of the low-temperature Sakai-Sugimoto model, JHEP 06 (2011) 092 [arXiv:1104.2291] [INSPIRE].

[8] A. Ballon-Bayona, K. Peeters and M. Zamaklar, A chiral magnetic spiral in the holographic Sakai-Sugimoto model, JHEP 11 (2012) 164 [arXiv:1209.1953] [INSPIRE].

[9] S.K. Domokos, C. Hoyos and J. Sonnenschein, Stability conditions for spatially modulated phases, JHEP 11 (2013) 172 [arXiv:1307.3773] [INSPIRE].

[10] W.-Y. Chuang, S.-H. Dai, S. Kawamoto, F.-L. Lin and C.-P. Yeh, Dynamical instability of holographic QCD at finite density, Phys. Rev. D 83 (2011) 106003 [arXiv:1004.0162] [INSPIRE].

[11] K.-Y. Kim, S.-J. Sin and I. Zahed, The chiral model of Sakai-Sugimoto at finite baryon density, JHEP 01 (2008) 002 [arXiv:0708.1469] [INSPIRE].

[12] K.-Y. Kim, B. Sahoo and H.-U. Yee, Holographic chiral magnetic spiral, JHEP 10 (2010) 005 [arXiv: 1007.1985] [INSPIRE].

[13] O. Bergman, G. Lifschytz and M. Lippert, Holographic nuclear physics, JHEP 11 (2007) 056 [arXiv:0708.0326] [INSPIRE].

[14] O. Bergman, G. Lifschytz and M. Lippert, Magnetic properties of dense holographic QCD, Phys. Rev. D 79 (2009) 105024 [arXiv:0806.0366] [INSPIRE]. 
[15] O. Aharony, K. Peeters, J. Sonnenschein and M. Zamaklar, $\rho$ meson condensation at finite isospin chemical potential in a holographic model for QCD, JHEP 02 (2008) 071 [arXiv: 0709.3948] [INSPIRE].

[16] O. Bergman, G. Lifschytz and M. Lippert, Response of holographic QCD to electric and magnetic fields, JHEP 05 (2008) 007 [arXiv:0802.3720] [INSPIRE].

[17] C.V. Johnson and A. Kundu, External fields and chiral symmetry breaking in the Sakai-Sugimoto model, JHEP 12 (2008) 053 [arXiv:0803.0038] [INSPIRE].

[18] F. Preis, A. Rebhan and A. Schmitt, Inverse magnetic catalysis in dense holographic matter, JHEP 03 (2011) 033 [arXiv: 1012.4785] [INSPIRE].

[19] N. Callebaut and D. Dudal, On the transition temperature(s) of magnetized two-flavour holographic QCD, Phys. Rev. D 87 (2013) 106002 [arXiv:1303.5674] [INSPIRE].

[20] K. Ghoroku, A. Nakamura and F. Toyoda, Stability of D-brane anti D-brane systems in confining gauge theories, Eur. Phys. J. C 71 (2011) 1522 [arXiv:0907.1170] [INSPIRE].

[21] O. Mintakevich and J. Sonnenschein, On the spectra of scalar mesons from HQCD models, JHEP 08 (2008) 082 [arXiv:0806.0152] [INSPIRE].

[22] M. Chernodub, Superconductivity of QCD vacuum in strong magnetic field, Phys. Rev. D 82 (2010) 085011 [arXiv:1008.1055] [INSPIRE].

[23] M. Chernodub, Spontaneous electromagnetic superconductivity of vacuum in strong magnetic field: evidence from the Nambu-Jona-Lasinio model, Phys. Rev. Lett. 106 (2011) 142003 [arXiv:1101.0117] [INSPIRE].

[24] V. Skokov, A.Y. Illarionov and V. Toneev, Estimate of the magnetic field strength in heavy-ion collisions, Int. J. Mod. Phys. A 24 (2009) 5925 [arXiv:0907.1396] [inSPIRE].

[25] W.-T. Deng and X.-G. Huang, Event-by-event generation of electromagnetic fields in heavy-ion collisions, Phys. Rev. C 85 (2012) 044907 [arXiv: 1201.5108] [INSPIRE].

[26] A. Bzdak and V. Skokov, Event-by-event fluctuations of magnetic and electric fields in heavy ion collisions, Phys. Lett. B 710 (2012) 171 [arXiv:1111.1949] [INSPIRE].

[27] K. Tuchin, Particle production in strong electromagnetic fields in relativistic heavy-ion collisions, Adv. High Energy Phys. 2013 (2013) 490495 [arXiv: 1301.0099] [INSPIRE].

[28] K. Tuchin, Time and space dependence of electromagnetic field in relativistic heavy-ion collisions, Phys. Rev. C 88 (2013) 024911 [arXiv:1305.5806] [INSPIRE].

[29] L. McLerran and V. Skokov, Comments about the electromagnetic field in heavy-ion collisions, arXiv:1305.0774 [INSPIRE].

[30] D.E. Kharzeev, K. Landsteiner, A. Schmitt and H.-U. Yee, 'Strongly interacting matter in magnetic fields': an overview, Lect. Notes Phys. 871 (2013) 1 [arXiv:1211.6245] [InSPIRE].

[31] O. Bergman, J. Erdmenger and G. Lifschytz, A review of magnetic phenomena in probe-brane holographic matter, Lect. Notes Phys. 871 (2013) 591 [arXiv:1207.5953] [INSPIRE].

[32] D. Djukanovic, M.R. Schindler, J. Gegelia and S. Scherer, Quantum electrodynamics for vector mesons, Phys. Rev. Lett. 95 (2005) 012001 [hep-ph/0505180] [INSPIRE].

[33] V. Braguta, P. Buividovich, M. Chernodub, A.Y. Kotov and M. Polikarpov, Electromagnetic superconductivity of vacuum induced by strong magnetic field: numerical evidence in lattice gauge theory, Phys. Lett. B 718 (2012) 667 [arXiv:1104.3767] [INSPIRE].

[34] M. Chernodub, J. Van Doorsselaere and H. Verschelde, Electromagnetically superconducting phase of vacuum in strong magnetic field: structure of superconductor and superfluid vortex lattices in the ground state, Phys. Rev. D 85 (2012) 045002 [arXiv:1111.4401] [INSPIRE]. 
[35] M. Chernodub, Electromagnetic superconductivity of vacuum induced by strong magnetic field, Lect. Notes Phys. 871 (2013) 143 [arXiv:1208.5025] [INSPIRE].

[36] M. Ammon, J. Erdmenger, P. Kerner and M. Strydom, Black hole instability induced by a magnetic field, Phys. Lett. B 706 (2011) 94 [arXiv:1106.4551] [INSPIRE].

[37] Y.-Y. Bu, J. Erdmenger, J.P. Shock and M. Strydom, Magnetic field induced lattice ground states from holography, JHEP 03 (2013) 165 [arXiv:1210.6669] [INSPIRE].

[38] R.-G. Cai, S. He, L. Li and L.-F. Li, A holographic study on vector condensate induced by a magnetic field, JHEP 12 (2013) 036 [arXiv: 1309.2098] [INSPIRE].

[39] Y. Hidaka and A. Yamamoto, Charged vector mesons in a strong magnetic field, Phys. Rev. D 87 (2013) 094502 [arXiv: 1209.0007] [inSPIRE].

[40] M. Chernodub, Vafa-Witten theorem, vector meson condensates and magnetic-field-induced electromagnetic superconductivity of vacuum, Phys. Rev. D 86 (2012) 107703 [arXiv:1209.3587] [INSPIRE].

[41] M. Chernodub, Comment on "charged vector mesons in a strong magnetic field", arXiv:1309.4071 [INSPIRE].

[42] A. Karch and E. Katz, Adding flavor to AdS/CFT, JHEP 06 (2002) 043 [hep-th/0205236] [INSPIRE].

[43] O. Bergman, S. Seki and J. Sonnenschein, Quark mass and condensate in HQCD, JHEP 12 (2007) 037 [arXiv:0708.2839] [INSPIRE].

[44] K. Hashimoto, T. Hirayama and A. Miwa, Holographic QCD and pion mass, JHEP 06 (2007) 020 [hep-th/0703024] [INSPIRE].

[45] K. Hashimoto, T. Hirayama, F.-L. Lin and H.-U. Yee, Quark mass deformation of holographic massless QCD, JHEP 07 (2008) 089 [arXiv:0803.4192] [INSPIRE].

[46] O. Aharony, J. Sonnenschein and S. Yankielowicz, A holographic model of deconfinement and chiral symmetry restoration, Annals Phys. 322 (2007) 1420 [hep-th/0604161] [INSPIRE].

[47] I. Shushpanov and A.V. Smilga, Quark condensate in a magnetic field, Phys. Lett. B 402 (1997) 351 [hep-ph/9703201] [INSPIRE].

[48] O. Aharony and D. Kutasov, Holographic duals of long open strings, Phys. Rev. D 78 (2008) 026005 [arXiv:0803.3547] [INSPIRE].

[49] A. Dhar and P. Nag, Sakai-Sugimoto model, tachyon condensation and chiral symmetry breaking, JHEP 01 (2008) 055 [arXiv:0708.3233] [INSPIRE].

[50] A. Dhar and P. Nag, Tachyon condensation and quark mass in modified Sakai-Sugimoto model, Phys. Rev. D 78 (2008) 066021 [arXiv:0804.4807] [InSPIRE].

[51] G.S. Bali and K. Schilling, Running coupling and the $\Lambda$ parameter from $\mathrm{SU}(3)$ lattice simulations, Phys. Rev. D 47 (1993) 661 [hep-lat/9208028] [INSPIRE].

[52] R. Sommer, A new way to set the energy scale in lattice gauge theories and its applications to the static force and $\alpha_{s}$ in $\mathrm{SU}(2)$ Yang-Mills theory, Nucl. Phys. B 411 (1994) 839 [hep-lat/9310022] [INSPIRE].

[53] R. Leigh, Dirac-Born-Infeld action from Dirichlet $\sigma$-model, Mod. Phys. Lett. A 4 (1989) 2767 [inSPIRE].

[54] E. Fradkin and A.A. Tseytlin, Nonlinear electrodynamics from quantized strings, Phys. Lett. B 163 (1985) 123 [INSPIRE]. 
[55] A.A. Tseytlin, On non-Abelian generalization of Born-Infeld action in string theory, Nucl. Phys. B 501 (1997) 41 [hep-th/9701125] [INSPIRE].

[56] R.C. Myers, Non-Abelian phenomena on D-branes, Class. Quant. Grav. 20 (2003) S347 [hep-th/0303072] [INSPIRE].

[57] P. Howe, U. Lindström and L. Wulff, On the covariance of the Dirac-Born-Infeld-Myers action, JHEP 02 (2007) 070 [hep-th/0607156] [INSPIRE].

[58] L. Wulff, Strings, boundary fermions and coincident D-branes, hep-th/0701129 [INSPIRE].

[59] A. Hashimoto and W. Taylor, Fluctuation spectra of tilted and intersecting D-branes from the Born-Infeld action, Nucl. Phys. B 503 (1997) 193 [hep-th/9703217] [INSPIRE].

[60] A. Sevrin, J. Troost and W. Troost, The non-Abelian Born-Infeld action at order $F^{6}$, Nucl. Phys. B 603 (2001) 389 [hep-th/0101192] [INSPIRE].

[61] E. Antonyan, J. Harvey, S. Jensen and D. Kutasov, NJLS and QCD from string theory, hep-th/0604017 [INSPIRE].

[62] P.C. Argyres, M. Edalati, R.G. Leigh and J.F. Vazquez-Poritz, Open Wilson lines and chiral condensates in thermal holographic QCD, Phys. Rev. D 79 (2009) 045022 [arXiv: 0811.4617] [INSPIRE].

[63] F. Denef, A. Sevrin and J. Troost, Non-Abelian Born-Infeld versus string theory, Nucl. Phys. B 581 (2000) 135 [hep-th/0002180] [InSPIRE].

[64] G. 't Hooft, Renormalizable Lagrangians for massive Yang-Mills fields, Nucl. Phys. B 35 (1971) 167 [InSPIRE].

[65] J. Erdmenger, K. Ghoroku and I. Kirsch, Holographic heavy-light mesons from non-Abelian DBI, JHEP 09 (2007) 111 [arXiv:0706.3978] [InSPIRE].

[66] T. Imoto, T. Sakai and S. Sugimoto, Mesons as open strings in a holographic dual of QCD, Prog. Theor. Phys. 124 (2010) 263 [arXiv: 1005.0655] [INSPIRE].

[67] A.F.J. Levi, Applied quantum mechanics, Cambridge University Press, Cambridge U.K. (2006).

[68] I. Obukhov, V. Peres-Fernandes, I. Ternov and V. Khalilov, Charged vector particles in a magnetic field, Theor. Math. Phys. 55 (1983) 536 [Teor. Mat. Fiz. 55 (1983) 335] [InSPIRE].

[69] V. Orlovsky and Y. Simonov, Nambu-Goldstone mesons in strong magnetic field, JHEP 09 (2013) 136 [arXiv: 1306.2232] [INSPIRE].

[70] J. Erdmenger, N. Evans and J. Grosse, Heavy-light mesons from the AdS/CFT correspondence, JHEP 01 (2007) 098 [hep-th/0605241] [INSPIRE].

[71] S. Bolognesi, F. Kiefer and E. Rabinovici, Comments on critical electric and magnetic fields from holography, JHEP 01 (2013) 174 [arXiv:1210.4170] [INSPIRE].

[72] S. Ferrara and M. Porrati, String phase transitions in a strong magnetic field, Mod. Phys. Lett. A 8 (1993) 2497 [hep-th/9306048] [INSPIRE].

[73] B.-H. Lee and M.C. Wapler, Three roads to probe-brane superconductivity, JHEP 02 (2011) 085 [arXiv:1011.5218] [INSPIRE].

[74] P. Mathews, Stationary states of a spin-1 particle in a homogeneous magnetic field, Phys. Rev. D 9 (1974) 365 [INSPIRE]. 